UNIVERSIDADE DE SÃO PAULO

INSTITUTO DE FÍSICA

\title{
Perturbações Gravitacionais e Propagação de Ondas em Buracos Negros com Campos Eletromagnéticos
}

\author{
Rodrigo Dal Bosco Fontana
}

Orientador: Prof. Dr. Elcio Abdalla

Tese de doutorado apresentada ao Instituto de Física da Universidade de São Paulo para a obtenção do título de Doutor em Ciências

Comissão Examinadora:

Prof. Dr. Elcio Abdalla (IF-USP)

Prof. Dr. João Carlos Alves Barata (IF-USP)

Prof. Dr. Marcos Vinicius Borges Teixeira Lima (IF-USP)

Prof. Dr. Alberto Vazquez Saa (UNICAMP)

Prof. Dr. George Emanuel Avraam Matsas (IFT-UNESP)

São Paulo 


\section{Resumo}

Neste trabalho faremos uma investigação no campo das perturbações gravitacionais e propagação de ondas em geometrias de buracos negros com campos elétricos ou magnéticos. Usando uma geometria tipo Ernst-Melvin de um buraco negro massivo em um Universo com campo magnético no eixo $z$, calculamos os modos quasi-normais de propagação de um campo escalar, demonstrando que este se comporta como um campo escalar com massa $2|m| B$ em uma geometria de Schwarzschild, para pequenos valores do campo magnético $B$ (correspondendo $m$ ao número azimutal do esférico harmônico). Ainda com esta geometria, calculamos a contribuição de ondas escalares para a entropia do buraco negro em termos dos cut offs ultravioleta e infravermelho. Com uma solução do tipo Reissner-Nordström em 4 dimensões, investigamos as possíveis correspondências entre os modos quasinormais e as propriedades termodinâmicas deste buraco negro, atestando o resultado de que a conjectura Hod modificada por Maggiore é válida em tal solução. Também, com uma geometria de Reissner-Nordström-de Sitter $D$-dimensional, obtivemos os modos quasi-normais de vibração para dois potenciais diferentes, estabelecendo a ausência de modos instáveis para uma grande gama de parâmetros deste buraco negro. 


\begin{abstract}
In this work we make an incursion into the branch of gravitational perturbations and field propagation around known-geometries of black holes with electromagnetic fields. Using an Ernst-Melvin type of geometry in a massive black hole immersed on a magnetic Universe, we calculate the quasi-normal modes of the propagating field, showing the equivalence of this problem with that of a massive scalar field (for which the mass is $2|m| B, m$ being the azhimutal number, and $B$ the magnetic field) propagating around a Schwarzschild geometry. We also compute the contribution of the scalar field to the entropy of the black hole in terms of the infrared and ultraviolet cut offs. Using a Reissner-Nordström-like solution in 4 dimensions, we investigate the possible correspondence between quasi-normal modes and the thermodynamical properties of this black hole, atesting the validity of the modified Hod conjecture as proposed by Maggiore. Finally, for a Reissner-Nordström-de Sitter D-dimensional solution, we obtain the quasi-normal modes for two diferent potentials, establishing the absence of unstable modes for a large range of values for the black hole parameters.
\end{abstract}




\section{Agradecimentos}

É certo que este trabalho não teria sido acabdo, não fossem as pessoas que cito aqui, e também algumas outras que eventualmente não cite por memória falha (para as quais dirijo minhas desculpas).

Devo começar agradecendo ao professor Dr. Elcio Abdalla pela orientação, amizade e enorme paciência durante todo o período do doutorado.

Ao professor Dr. Kostas Kokkotas pelo acolhimento, orientação e também pela paciência durante o período de doutorado sanduíche.

Agradeço aos amigos e companheiros, sem os quais nada disso teria se consumado. Por medo de acabar esquecendo algum nome que deva citar aqui, faço como Clarice Lispector e me esquivo de citar seus nomes (também pedindo seu entendimento a este respeito).

Aos colegas de trabalho da sala 319, Alan Pavan, Jeferson de Oliveira, Carlos Eduardo e a Juliano Neves, por longas conversas, por uma convivência amistosa e boas discussões sobre física.

Aos colegas e colaboradores (e tutores!) Roman Konoplya e Olexandr Zhidenko, pela grande ajuda e colaboração do começo ao fim desta tese.

A Ivanildo Antonio dos Santos, que fez a parte final deste trabalho possível, não há papel suficiente para os agradecimentos.

À FAPESP pelo apoio financeiro durante o período do doutorado, e ao $\mathrm{CNPq}$ pelo também apoio financeiro para o doutorado sanduíche.

Finalmente, a meu familiares, que estão todos os dias comigo, de longe ou de perto: minha mãe Claci, meu pai Carlos, e minha irmã Duda. 


\section{Sumário}

1 Introdução $\quad 6$

2 Buracos Negros com Campos Magnéticos 13

2.1 Transformações de Harrison: novas soluções a partir de antigas 14

2.2 Campos Magnéticos: Os Universos de Melvin e Ernst . . . . . 19

2.3 Campos Escalares na Métrica de Ernst: os Modos Quasi-normais 23

2.4 A Entropia do Buraco Negro de Ernst: contribuição com Campo

Escalar . . . . . . . . . . . . . . . . . . 31

2.4.1 O buraco negro dilatônico . . . . . . . . . . . 37

2.4.2 O caso $D$-dimensional $\ldots \ldots \ldots \ldots$

3 Modos Quasi-Normais e Termodinâmica em Buracos Negros 46

3.1 A Conjectura Hod . . . . . . . . . . . . . . . . 47

3.2 A Conjectura Hod Modificada . . . . . . . . . . . . . . . . . . 49

3.3 Testando a conjectura com Buracos Negros de Reissner-Nordström 55

3.4 Perturbações em Reissner-Nordström . . . . . . . . . . . . . . 59

3.4.1 Perturbação Gravitacional . . . . . . . . . . . . . . 60

3.4.2 O método de Leaver (Frobenius) - frações parciais . . . 63

3.4.3 Propagação dos Campos de Spin meio e Escalar na geometria de Reissner-Nordström . . . . . . . . . . 66

3.5 Mudança de Fase de Segunda Ordem em Buracos Negros e Modos Quasi-normais . . . . . . . . . . . . 70

3.5.1 O Comportamento de $\omega_{0} \ldots \ldots \ldots 77$ 
4 Modos Quasi-Normais em Buracos Negros Cosmológicos $\quad 79$

4.1 Perturbações Gravitacionais Tensoriais . . . . . . . . . . . . . 81

4.1.1 Método de Integração em Coordenadas Nulas . . . . . 83

4.1.2 Método Prony . . . . . . . . . . . . . . . . . . 85

4.2 Análise de Horizontes . . . . . . . . . . . . . . . . . . 87

4.3 Modos Quasi-normais com a Perturbação Tensorial . . . . . . 90

4.4 Perturbação Vetorial . . . . . . . . . . . . . . . . . . . . . . . 98

4.5 Resultados com a Perturbação Vetorial . . . . . . . . . . . . . 100

5 Apontamentos Finais 106

$\begin{array}{ll}\text { Referências Bibliográficas } & 109\end{array}$ 


\section{Capítulo 1}

\section{Introdução}

Buracos negros são objetos de estudo da astronomia e da física teórica há um tempo considerável. A primeira "pré-aparição" do conceito de buraco negro no contexto da relatividade geral surgiu em 1916 quando Karl Schwarzschild resolveu as equações de campo de Einstein usando de simetria esférica para obter a descrição gravitacional de um sistema gravitacional com massa ${ }^{1}$. Embora o próprio Schwarzschild não tenha vislumbrado que a solução - nomeada solução de Schwarzschild postumamente em seu crédito - poderia representar um espaço-tempo que sob certas circunstâncias aprisione até mesmo a luz, algumas décadas mais tarde tal interpretação passou a ser cogitada pela astronomia.

A existência de um objeto de porte astronômico condensado em um espaço extremamente pequeno, que gerasse um campo de atração tão intenso a ponto

\footnotetext{
${ }^{1}$ Há que se citar contudo que pelo menos um século antes, Laplace considerou em seus cálculos sobre mecânica celeste um objeto astrofísico cuja densidade seria tão alta, que até mesmo a luz seria aprisionadade em seu campo gravitacional. Em tal situação, o limite entre objetos ligados ou não (gravitacionalmente) é quando a energia mecânica é nula, $E=0$. Com isto, podemos obter, "classicamente" (sem relatividade geral) o raio de Schwarzschild, que corresponde à tomar a velocidade da luz na expressão da energia cinética,$$
E=T+P=\frac{m c^{2}}{2}-\frac{G M m}{r}=0
$$

O ponto $r=2 G M / c^{2}$ corresponde neste caso, à região esférica limite: se houvesse uma massa $M$ aprisionada nesta esfera, classicamente, nem mesmo a luz escaparia gravitacionalmente do potencial.
} 
de aprisionar a luz e ainda que não resistisse à sua própria força gravitacional foi, em realidade, preterida pelo próprio Einstein alguns anos mais tarde depois do aparecimento da solução de Schwarzschild.

Embora em seu início, a existência de buracos negros como objetos astronômicos de nosso Universo tenha sido rejeitada, há nos dias de hoje um grande número de evidências observacionais que atestam de maneira indireta a presença destes objetos em regiões distintas do cosmos.

Uma evidência experimental robusta para a existência de buracos negros seria a medida de ondas gravitacionais em grandes laboratórios como LIGO [1] ou VIRGO [2], ou ainda o detector de ondas gravitacionais Schenberg [3]. Ainda que a evidência de sinais de ondas gravitacionais não constituam uma prova definitiva à existência de tais objetos, uma vez que o espectro gravitacional deles é diferente de outros objetos como estrelas, a observação do sinal gravitacional pode ser, em tese, diferenciado de maneira a atribuir uma evidência robusta a mais para a existência de tais objetos.

Uma vez atestada a existência dos buracos negros, mesmo que indiretamente através da medida de ondas gravitacionais, há, concomitantemente, na física teórica, uma extensa literatura de perturbações gravitacionais já consolidada. Um dos trabalhos pioneiros testando a estabilidade da primeira solução de buracos negros publicado em 1955 deve-se a Regge e Wheeler [4].

Neste trabalho, os autores analisam o efeito que pequenas perturbações na geometria de Schwarzschild podem gerar em termos dinâmicos à sua métrica, terminando por concluir que o buraco negro é estável ${ }^{2}$. O artigo é de fundamental importância por ter sido o primeiro a introduzir uma decomposição do tensor perturbação gravitacional, de acordo com a simetria do espaço-tempo esfericamente simétrico. Depois de uma decomposição genérica o suficiente em harmônicos esféricos tensoriais que respeitassem certas simetrias, os autores consideram ainda as simetrias de Killing do espaço-tempo, chegando a demonstrar que a equação de perturbação gravitacional poderia ser colocada

\footnotetext{
${ }^{2}$ Cabe notar porém que à época o que era chamado "buraco negro" de Schwarzschild, referia-se ao horizonte de eventos deste buraco negro ("a esfera de Schwarzschild"). Foi só mais tarde, com o surgimento do diagrama de Kruskal que a questão da continuidade para além da esfera do horizonte de eventos foi resolvida, embora os cálculos feitos por Regge e Wheeler à respeito da estabilidade do horizonte de eventos de Schwarzschild sejam válidos.
} 
em um formato relativamente simples análogo ao de uma equação de onda,

$$
-\frac{\partial^{2}}{\partial t^{2}} \chi+\frac{\partial^{2}}{\partial x^{2}} \chi+V(x) \chi=0
$$

sendo $x$ uma coordenada espacial, $t$ uma coordenada temporal e $V(x)$ o potencial gravitacional. O formato de $V(x)$ depende essencialmente do tipo de simetria usado para a decomposição do tensor perturbação gravitacional.

Além de perturbações gravitacionais de diversas geometrias cuja equação de perturbação é como (1.2), também campos de partículas de spins diferentes seguem a mesma equação, com pequenas variações apenas no potencial $V(x)$, tais como os campos escalar e eletromagnético. Embora a propagação de um campo de partículas em uma dada geometria não possa ser entendida do ponto de vista físico como um teste fidedigno para a estabilidade da dada geometria, a análise das equações de propagação de tais tipos de campo pode prover um preâmbulo sobre a estabilidade do espaço-tempo: uma vez que os modos de vibração resultantes de tais equações sejam estáveis (i. e. nulos no infinito espacial e quadrado-integráveis), pode-se demonstrar que isto corresponderia à estabilidade de pequenas perturbações no próprio espaço-tempo.

Neste sentido, podemos usar a equação do campo escalar, por exemplo, para testar a presença de modos estáveis para uma dada geometria. Fisicamente isto corresponderia a uma perturbação (pequena) da geometria, cuja resposta emerge como um campo que decai como uma senóide amortecida com o tempo.

O sinal da onda gerada, seja pela propagação de um campo em uma geometria fixa, seja por uma pequena perturbação gravitacional em um espaçotempo "estável" 3 pode ser entendido como a resposta da geometria à propagação do campo, ou à perturbação, e, sendo expresso em termos de ondas amortecidas, formam um conjunto nomeado "modos quasi-normais" [5].

Os modos quasi-normais representam um grupo de vibrações para uma

\footnotetext{
${ }^{3} \mathrm{O}$ conceito de estabilidade do espaço-tempo tanto à propagação de campos quanto à perturbação está relacionado com a ausência de modos de vibração que não possuam uma das duas características supracitadas, e. g. modos que ao invés de decaimento, expressem um crescimento exponencial.
} 
dada solução de buracos negros que não formam um conjunto completo, no sentido de que o grupo de todas as frequências amortecidas podem formar uma soma que se aproxima da auto-função da equação de onda em determinadas regiões do espaço,

$$
\chi \sim \sum_{n=1}^{N} a_{n} e^{-\left(\omega_{I}^{(n)}+i \omega_{R}^{(n)}\right) t} f\left(\omega_{n}, x\right)
$$

mas não representa uma auto-função para todo o domínio da coordenada temporal, como as auto-funções de sistemas dinâmicos oscilantes de mecânica.

Assim como os modos de vibração de uma corda são ordenados de acordo com o harmônico de vibração $n$, de maneira que quanto maior $n$, maior é a frequência de vibração da corda, também as vibrações em sistemas gravitacionais com "quasi-soluções" (soluções localizadas em um determinado intervalo de tempo) podem ser ordenados, mas em relação ao parâmetro de decaimento do modo, $\omega_{I}$, de maneira que quanto maior $n$, maior o valor de $\omega_{I}$, ou seja, maior o amortecimento.

No próximo capítulo deste trabalho faremos uma investigação dos modos quasi-normais de buracos negros com campo magnético no eixo $z$. Trata-se de uma geometria de buraco negro com massa imerso em um Universo com um campo magnético de fundo. Fisicamente tal situação tem importância pelo fato de que, em geral, buracos negros sem rotação (ou com pequena rotação) têm ao seu redor discos de matéria que geram um campo magnético perpendicular à esfera de Schwarzschild. A diferença entre uma solução de Ernst que representa um buraco negro de Schwazschild com campo magnético e uma situação realista é o comportamento do espaço-tempo no infinito: em tal solução, o campo magnético tem a mesma intensidade no infinito e próximo ao horizonte. Desta maneira, por uma questão de aproximação com a situação física, é de maior interesse desconsiderar que o espaço tenha um campo magnético assintótico, e, considerar que a geometria assintótica seja a de Schwarzschild.

Na segunda parte do capítulo dois, focamos o estudo da contribuição do campo escalar para a entropia do buraco negro com campo magnético, usando 
do modelo Brick-Wall como proposto na década de 80 por 't Hooft. O cálculo demonstra uma contribuição não nula para as divergências infra-vermelha e ultra-violeta geradas pelo campo eletromagnético em relação a entropia do campo escalar. O ponto de cut off em tal caso deixa de ser um invariante (interpretado como uma renormalização da constante gravitacional Newtoniana) e traz uma contribuição do campo proporcional à sua magnitude.

Instabilidades gravitacionais em soluções das equações de Einstein com dadas simetrias estão associadas em muitas geometrias com a presença de modos para os quais $\omega_{I}<0$, ou, equivalentemente, para os quais a exponencial cresce ao invés de decair $[6,7,8,9]$.

Além da relação com a estabilidade geométrica de uma dada solução, os modos quasi-normais estão, em certo sentido, relacionados com as propriedades termodinâmicas de buracos negros.

O estudo da termodinâmica dos buracos negros teve seu início e período mais fértil na década de 70 com os trabalhos publicados por Hawking e Bekenstein [10, 11]. Dois notáveis resultados emergentes de tais trabalhos são a criação de pares de partículas nas proximidades do horizonte de eventos de buracos negros e posterior evaporação destes, e a associação da entropia do buraco negro com a área de seu horizonte de eventos,

$$
S=\frac{A_{H}}{4}
$$

A criação de pares partícula/anti-partícula na borda do horizonte de buracos negros, sugerida inicialmente por Hawking, e nomeada homonimamente radiação Hawking pode ser entendida do ponto de vista de um observador externo ao buraco negro, para o qual partículas que seguem algumas das geodésicas internas ao horizonte de eventos possuem energia negativa, o que possibilita a interpretação de que o buraco negro irradia em sua direção.

Além da entropia atribuída ao buraco negro proporcional à área de seu horizonte de eventos, também atribuímos uma capacidade térmica $C$ ao buraco negro, $C=T \frac{\partial S}{\partial T}$, que, dependendo dos parâmetros da solução pode ser ou negativa ou positiva. A transição entre estes ramos de valores é dada por 
uma "mudança de fase de segunda ordem".

O terceiro capítulo deste trabalho é dedicado, entre outros cálculos à análise da relação entre esta transição de fase de segunda ordem para o buraco negro de Reissner-Nordström. Investigamos uma possível associação entre curvas descontínuas nas oscilações quasi-normais e a capacidade térmica deste buraco negro. O fato de que em buracos negros cuja capacidade calorífica não tenha uma transição de segunda ordem (e. g. o buraco negro de Schwarzschild) e, concomitantemente de, os modos quasi-normais terem frequência natural $\omega_{0}=\sqrt{\omega_{R}^{2}+\omega_{I}^{2}}$ crescente, conforme a variação de seus parâmetros, pode ser o indicativo de que tais oscilações estejam conectadas com propriedades termodinâmicas do buraco negro de alguma maneira ainda não descrita. Na segunda parte do terceiro capítulo deste trabalho investigamos esta possível conexão usando como teste a solução com carga e massa, para a qual existe uma transição de segunda ordem a $87 \%$ do valor máximo de carga permitido para este buraco negro.

Também neste capítulo investigamos a possível reinterpretação das frequências quasi-normais em comparação com um sistema mecânico simples tal como o oscilador harmônico com atrito, proposta recente para uma reinterpretação da conjectura Hod de quantização da área do buraco negro. Com esta reinterpretação, pode-se expressar uma resposta coerente a algumas das antigas críticas da conjectura. Nas primeiras seções, investigamos o espaçamento entre os modos quasi-normais de Reissner-Nordström para sugerir uma resposta sobre se a proposta é válida pra outros buracos negros que não apenas o de Schwarzschild.

No último capítulo deste trabalho investigamos as vibrações quasi-normais de um buraco negro de Reissner-Nordström em 5 dimensões com constante cosmológica positiva. Apesar da existência de modos instáveis para tal geometria em quando o número de dimensões for maior do que 6, com um potencial escalar, verificamos que todas as frequências calculadas representam pequenas oscilações em torno da métrica, que decaem com o tempo de maneira exponencial em 5 dimensões. Com o uso de dois potenciais diferentes, a saber, vetorial e tensorial, ambos gravitacionais, advindos da decomposição equivalente do tensor perturbação gravitacional, calculamos as frequências 
quasi-normais para uma diferente gama de valores dos parâmetros envolvidos na solução, $M, Q$ e $\Lambda$.

O comportamento oscilatório de tais buracos negros depende destes parâmetros, ou, equivalentemente, da posição de cada horizonte considerado. De maneira geral, quanto "maior" a distância entre os horizontes de eventos e cosmológico (medida no sistema de coordenadas radial), maior é a amplitude de frequências quasi-normais possíveis, para um dado valor da constante cosmológica.

Outro fator de influência nas frequências é o valor do momento angular das ondas incidentes no potencial. Tal influência, para geometrias esfericamente simétricas, aparece no potencial com coeficiente $l(l+D-3)$, sendo $l$ o número azimutal de "momento angular" e $D$ a dimensão do espaço-tempo. No geral quanto maior o valor do momento angular, maior o valor da parte real do modo, variando a parte imaginária de um fator muito menor.

Nos próximos capítulos, procurou-se em cada ocasião introduzir o assunto com a teoria já desenvolvida em literatura específica de cada caso, de maneira a fazer deste trabalho auto-consistente. 


\section{Capítulo 2}

\section{Buracos Negros com Campos Magnéticos}

Buracos negros magnéticos são objetos de estudo da relatividade geral com destacado interesse em astrofísica. Sabemos hoje que, em geral, uma solução exata das equações de Einstein para um buraco negro em 4 dimensões não pode representar todas as situações reais observadas pela astronomia. Buracos negros, via de regra, estão envolvidos em nuvens de poeira, que modificam o espaço-tempo ao seu redor, produzindo uma contribuição não nula à geometria. Entretanto, esta contribuição não tem a mesma ordem de grandeza da massa do buraco negro. Por exemplo, para buracos negros astrofísicos, a contribuição do campo magnético gerado pela nuvem de poeira é da ordem de $10^{-4}$ vezes a massa do buraco negro. Um valor semelhante pode ser acrescido devido às forças de maré desta nuvem.

O intuito deste trabalho é estudar tais tipos de objetos magnéticos, em que modificamos a geometria de uma solução conhecida da relatividade geral por meios como perturbações lineares da métrica, ou via transformações em seus coeficientes, para as quais a nova métrica represente uma nova solução das equações de Einstein.

Um exemplo de tal tipo de solução, é aquela obtida por Ernst [13] em 1976, em que se faz uma "rotação" nos potenciais que geram as equações de Einstein, obtendo-se uma nova solução com campo magnético, que não 
é assintóticamente plana. Começaremos o estudo desta solução, através dos trabalhos de Ernst e de Harrison [14], na próxima seção.

Em uma etapa futura, planejamos a investigação dos trabalhos de Preston e Poisson [15] que propõem uma nova solução, em primeira ordem nas equações de Einstein, com campo magnético e forças de maré, iniciada tomandose a métrica de Schwarzschild e adicionando a ela uma pequena perturbação.

\subsection{Transformações de Harrison: novas soluções a partir de antigas}

Em 1968 Harrison propôs, através de uma série de transformações nos coeficientes das equações de Einstein de uma dada métrica, uma maneira, de gerar novas soluções a partir de soluções já conhecidas. Daremos a seguir uma descrição básica do processo, necessário, por exemplo para a dedução da métrica de Ernst, um dos focos deste trabalho. De início, tomemos um elemento de linha dado por

$$
d s^{2}=-\epsilon\left[e^{2 U}\left(d x^{k}+a f_{\alpha} d x^{\alpha}\right)^{2}+a^{2} e^{-2 U} \gamma_{\alpha \beta} d x^{\alpha} d x^{\beta}\right],
$$

em que $\epsilon$ é o sinal do elemento $g_{k k}, a$ e $U$ são funções genéricas. Nesta notação, índices gregos podem assumir 3 valores (0,1 e 2), e latinos 4 (0,1,2 e 3), e $k$ é um índice específico de uma coordenada que representa um vetor de Killing da métrica: $\mathbb{L}_{g} \partial_{k}=0$. Nas definições acima, $\gamma$ é a 3-métrica, cuja conexão designaremos por $\Sigma$ e cujo tensor de Ricci será designado por $P$.

Nestas condições, o tensor de Ricci pode ser diretamente calculado com suas componentes dadas por

$$
\begin{array}{r}
R_{k k}=a^{-2}\left[e^{-2 U} \Delta_{2}(U)+e^{8 U} \gamma^{\alpha \beta} \gamma^{\gamma \delta} f_{(\alpha \gamma)} f_{(\beta \delta)}\right] \\
R_{k \alpha}=a f_{\alpha} R_{k k}+a^{-1} e^{4 U} \gamma^{\delta \gamma}\left(f_{(\delta \alpha), \gamma}+4 f_{(\delta \alpha)} U_{, \gamma}\right) \\
R_{\alpha \beta}=2 a f_{[\alpha} R_{k \beta]}-a^{2} f_{\alpha} f_{\beta} R_{k k}+P_{\alpha \beta}-2 U_{, \alpha} U_{, \beta} \\
+\gamma_{\alpha \beta} \Delta_{2}(U)-2 e^{4 U} \gamma^{\gamma \delta} f_{(\alpha \gamma)} f_{(\beta \delta)},
\end{array}
$$


em que utilizamos a notação de simetria para parêntesis e antisimetria para colchetes, e definimos ainda $\Delta_{2}(A)=\gamma^{\alpha \beta} A_{; \alpha \beta}$. Levando-se em conta agora o conteúdo de matéria que colocaremos no espaço-tempo, tomemos as equações de Maxwell sem fontes, e nas quais o tensor de Maxwell não depende de $x^{k}$,

$$
\begin{gathered}
{\left[\sqrt{-g} F^{k \alpha}\right]_{, \alpha}=0} \\
{\left[\sqrt{-g} F^{\beta \alpha}\right]_{, \alpha}=0} \\
F_{\alpha k, \beta}+F_{k \beta, \alpha}=0 \\
\varepsilon^{\alpha \beta \gamma} F_{\alpha \beta, \gamma}=0 .
\end{gathered}
$$

Uma possível solução para as equações (2.6) e (2.7) é dada em termos de potenciais $A$ e $B$,

$$
\begin{array}{r}
F^{\alpha \beta}=\frac{\varepsilon^{\alpha \beta \gamma} A_{, \gamma}}{\sqrt{-g}} \\
F_{k \alpha}=B_{, \alpha}
\end{array}
$$

que substituídos em (2.5) e (2.8) fornecem

$$
\begin{aligned}
& \Delta_{2}(A)-2 \Delta_{1}(U, A)+\frac{e^{2 U} \varepsilon^{\alpha \beta \gamma} f_{(\alpha \beta)} A_{, \gamma}}{\sqrt{-\gamma}}=0, \\
& \Delta_{2}(B)-2 \Delta_{1}(U, B)-\frac{e^{2 U} \varepsilon^{\alpha \beta \gamma} f_{(\alpha \beta)} B_{, \gamma}}{\sqrt{-\gamma}}=0,
\end{aligned}
$$

com $\Delta_{1}(A, B)=\gamma^{\alpha \beta} A_{, \alpha} B_{, \beta}$. Depois de computar devidamente o tensor momento energia em termos das quantidades $A$ e $B$ e dos coeficientes da métrica, podemos escrever as equações de Einstein como

$$
\begin{array}{r}
\Delta_{2}(U)-e^{4 U} \gamma^{\alpha \beta} \gamma^{\gamma \delta} f_{(\alpha \gamma)} f_{(\beta \delta)}+\epsilon e^{-2 U}\left[\Delta_{1}(A)+\Delta_{1}(B)\right]=0, \\
\gamma^{\delta \gamma}\left(f_{(\delta \alpha) ; \gamma}+4 f_{(\delta \alpha)}\right)-\frac{4 \epsilon \varepsilon^{\beta \gamma \delta} \gamma_{\alpha \beta} A_{, \delta} B_{, \gamma}}{e^{4 U} \sqrt{-\gamma}}=0, \\
P_{\alpha \beta}-2 U_{, \alpha} U_{, \beta}+e^{4 U} \gamma^{\gamma \delta}\left(\gamma_{\alpha \beta} \gamma^{\omega \eta} f_{(\gamma \omega)} f_{(\delta \eta)}-2 f_{(\alpha \gamma)} f_{\beta \delta}\right) \\
-2 \epsilon e^{-2 U}\left(A_{, \alpha} A_{, \beta}+B_{, \alpha} B_{, \beta}\right)=0 .
\end{array}
$$

$\left(\Delta_{1}(A) \equiv \Delta_{1}(A, A)\right)$. Agora, introduzimos um vetor axial $z$ e o potencial $\phi$ 
como

$$
\begin{array}{r}
2 f_{[\alpha \beta]}=\varepsilon_{\alpha \beta \gamma} \gamma^{\gamma \delta} z_{\delta} \sqrt{-\gamma} \\
\phi_{, \alpha}=e^{4 U} z_{\alpha}-2 \epsilon\left(B A_{, \alpha}-A B_{, \alpha}\right) .
\end{array}
$$

Redefinimos os potenciais do tensor energia-momento $A$ e $B$ em termos de novos potenciais $R$ e $\theta$,

$$
\begin{aligned}
& A=R \cos \theta \\
& B=R \operatorname{sen} \theta
\end{aligned}
$$

Com este preâmbulo podemos escrever seis equações de Einstein, a partir das quais trabalharemos na geração de novas soluções, a saber,

$$
\begin{array}{r}
z_{\alpha}=e^{-4 U}\left(\phi_{, \alpha}-2 \epsilon R^{2} \theta_{, \alpha}\right) \\
\Delta_{2}(\phi)-4 \Delta_{1}(U, \phi)+4 \epsilon R^{2} \Delta_{1}(U, \phi) \\
+2 R e^{-2 U}\left[2 R^{2} \Delta_{1}(R, \theta)-\epsilon \Delta_{1}(\phi, R)\right]=0 \\
\Delta_{2}(R)-R \Delta_{1}(\theta)-2 \Delta_{1}(U, R) \\
-R e^{-2 U}\left[\Delta_{1}(\phi, \theta)-2 \epsilon R^{2} \Delta_{1}(\theta)\right]=0 \\
R \Delta_{2}(\theta)+2 \Delta_{1}(R, \theta)-2 R \Delta_{1}(U, \theta) \\
-e^{-2 U}\left[\Delta_{1}(\phi, R)-2 \epsilon R^{2} \Delta_{1}(R, \theta)\right]=0 \\
2 \Delta_{2}(U)+2 \epsilon e^{-2 U}\left[\Delta_{1}(R)+R^{2} \Delta_{1}(\theta)\right] \\
+e^{-4 U}\left[\Delta_{1}(\phi)-4 \epsilon R^{2} \Delta_{1}(\phi, \theta)+4 R^{4} \Delta_{1}(\theta)\right]=0 \\
4 U_{, \alpha} U_{, \beta}+4 \epsilon e^{-2 U}\left(R_{, \alpha} R_{, \beta}+R^{2} \theta_{, \alpha} \theta_{, \beta}\right) \\
-2 P_{\alpha \beta}+e^{-4 U}\left(\phi_{, \alpha}-2 \epsilon R^{2} \theta_{, \alpha}\right)\left(\phi_{, \beta}-2 \epsilon R^{2} \theta_{, \beta}\right) .
\end{array}
$$

Para estas equações, o vácuo pode ser representado como, por exemplo $A=B=0$ ou $R=0 .{ }^{1}$ Depois de listadas as equações de Einstein, podemos supor uma nova solução em termos da antiga, apenas trocando as funções da métrica por outras equivalentes (por exemplo, $a$ por $\bar{a}, U$ por $\bar{U})$, mantendo-se a "métrica" tridimensional $\gamma$. As soluções das equações de

\footnotetext{
${ }^{1} \mathrm{O}$ tensor energia-momento com relação aos potenciais $A$ e $B$ e aos coeficientes da
} 
Einstein continuam, neste caso, como em (2.20-2.25). De modo semelhante, fazemos esta troca para os potenciais $A$ e $B$ do tensor energia-momento (ou $R$ e $\theta)$. O último passo é supor que estas funções com barra são funções das variáveis sem barra que não são constantes. Não é difícil mostrar, neste caso, que o parâmentro $\Delta_{1}$ de uma função com barra é linear. Por exemplo, sendo $\bar{R}=f(\phi, \theta)$, temos $\Delta_{1}(\bar{R})=\left[\frac{\partial \bar{R}}{\partial \phi}\right]^{2} \Delta_{1}(\phi)+2 \frac{\partial \bar{R}}{\partial \theta} \frac{\partial \bar{R}}{\partial \phi} \Delta_{1}(\theta, \phi)+\left[\frac{\partial \bar{R}}{\partial \theta}\right]^{2} \Delta_{1}(\theta) . \mathrm{O}$ procedimento para se resolver as novas equações de Einstein é achar equações em $\Delta_{1}$ e tornar o operador que o precede igual a zero.

Tomemos como exemplo o caso de Schwarzschild. No elemento de linha (2.1), supomos que $x^{k}=t$. Neste caso, temos necessariamente que $f_{\alpha}=0$, e $\epsilon e^{2 U}=1-\frac{2 M}{r}$. Como $\epsilon$ é apenas o sinal de $g_{t t}$, sua escolha é arbitrária. Ainda, podemos escolher $a=1$ e $\gamma_{\alpha \beta}=e^{2 U} \operatorname{diag}\left[e^{-2 U}, r^{2}, r^{2} \sin ^{2} \theta\right]$. Como a solução tem tensor momento-energia nulo, os potenciais $A=B=0$, o que nos leva a $R=0$ pelas equações (2.18-2.19). Ainda, a escolha de $\theta$ neste caso se torna arbitrária, e podemos tomar $\theta=0$ sem perda de generalidade. Como $z$ está definido em termos de $f(2.16)$ e esta variável é nula, temos também que $z=0$, do que segue imediatamente, por (2.20), que $\phi=0$. Assim, no caso de Schwarzschild todas os "coeficientes" das equações de Einstein são nulos, exceto $U$.

Uma nova solução seria gerada, tomando outros coeficientes nas equações de Einstein, com a métrica orginal: $\bar{\phi}, \bar{R}, \bar{\theta}, \bar{U}$.

A estratégia é (como descrito acima) igualar $\Delta_{1}(\bar{U})=\left[\frac{\partial \bar{U}}{\partial U}\right]^{2} \Delta_{1}(U)$ em todas as funções com barra nas equações dadas. Para obter as expressões de $\Delta_{2}$, tomamos as equações originais: $\Delta_{2}(F)=0$ para qualquer função $F$ da

métrica é escrito como segue,

$$
\begin{gathered}
T_{k k}=\frac{\epsilon e^{2 U}}{8 \pi a^{2}}\left[\Delta_{1}(A)+\Delta_{1}(B)\right] \\
T_{k \alpha}=a f_{\alpha} T_{k k}+\frac{\epsilon \varepsilon^{\beta \gamma \delta} \gamma_{\alpha \beta} A_{, \delta} B_{, \gamma}}{4 \pi a \sqrt{-\gamma}} \\
T_{\alpha \beta}=a f_{\alpha} T_{k \beta}+a f_{\beta} T_{k \alpha}-a^{2} f_{\alpha} f_{\beta} T_{k k} \\
+\frac{\epsilon e^{-2 U}}{8 \pi}\left\{2\left(A_{, \alpha} A_{, \beta}+B_{, \alpha} B_{, \beta}\right)-\gamma_{\alpha \beta}\left[\Delta_{1}(A)+\Delta_{1}(B)\right]\right\} .
\end{gathered}
$$


métrica ou do tensor energia-momento (e de fato esta relação é identicamente nula para qualquer coeficiente, exceto $U$, pois estes coeficientes são nulos). Assim, também teremos $\Delta_{2}(\bar{F})=0$. Levando em consideração ainda, que $\Delta_{1}(\bar{U}, \bar{\phi})=\frac{\partial \bar{U}}{\partial U} \frac{\partial \bar{\phi}}{\partial U} \Delta_{1}(U)$, com relações semelhantes para as demais funções $\Delta_{1}$, podemos escrever a segunda equação modificada de Einstein como

$$
\begin{array}{r}
\left\{-4 \frac{\partial \bar{U}}{\partial U} \frac{\partial \bar{\phi}}{\partial U}+4 \bar{\epsilon}^{2} \frac{\partial \bar{U}}{\partial U} \frac{\partial \bar{\theta}}{\partial U}\right. \\
\left.+2 \bar{R} e^{-2 \bar{U}}\left[2 \bar{R}^{2} \frac{\partial \bar{R}}{\partial U} \frac{\partial \bar{\theta}}{\partial U}-\bar{\epsilon} \frac{\partial \bar{\phi}}{\partial U} \frac{\partial \bar{R}}{\partial U}\right]\right\} \Delta_{1}(U)=0 .
\end{array}
$$

Podemos obter outras 4 equações não-triviais de (2.21-2.25). Assim, se o número de funções sem barra não constantes é $n$, teremos $5 n(n+1) / 2$ equações não triviais.

Através destas transformações, podemos deduzir teoremas de geração de novas soluções a partir de antigas. Abordaremos aqui um destes teoremas, a partir de uma solução de vácuo.

Tomemos, de início uma métrica com $U=R=0$, portanto com tensor energia-momento nulo. As equações de Einstein para o potencial $\phi$ quando redefinido em termos de um novo potencial $\bar{\phi}=\psi$ nesta métrica são dadas por

$$
\begin{gathered}
\Delta_{1}(\psi)=\Delta_{2}(\psi)=0, \\
1=4\left[\frac{d U}{d \psi}\right]^{2}+4 \epsilon e^{-2 U}\left[\left(\frac{d R}{d \psi}\right)^{2}+\left(R \frac{d \theta}{d \psi}\right)^{2}\right]+e^{-4 U}\left[\frac{d \phi}{d \psi}-2 \epsilon R^{2} \frac{d \theta}{d \psi}\right]^{2}(2
\end{gathered}
$$

Com isto podemos enunciar um teorema genérico, como segue.

Seja um espaço-tempo com um vetor de Killing $\partial_{k}$ definido por

$$
d s^{2}=-\epsilon\left[\left(d x^{k}+f_{\alpha} d x^{\alpha}\right)^{2}+\gamma_{\alpha \beta} d x^{\alpha} d x^{\beta}\right]
$$

$\operatorname{com} f_{\alpha}$ definido pelo potencial $\psi$,

$$
f_{(\alpha, \beta)}=2 \varepsilon_{\alpha \beta \gamma} \gamma^{\gamma \delta} \psi_{, \delta} \sqrt{-\gamma}
$$


Então existe uma solução generalizada envolvendo campos eletromagnéticos dada por

$$
d s^{2}=-\epsilon\left[e^{2 U}\left(d x^{k}+\bar{f}_{\alpha} d x^{\alpha}\right)^{2}+a^{2} e^{-2 U} \gamma_{\alpha \beta} d x^{\alpha} d x^{\beta}\right]
$$

em que $\bar{f}$ se relaciona com o novo potencial $\bar{\psi}$ da mesma maneira que em (2.30) e $\bar{\psi}$ é dado por

$$
\partial_{\beta} \bar{\psi}= \pm e^{-2 U}\left[\sqrt{1-4 U_{\psi}^{2}-2 \epsilon e^{-2 U}\left(R_{\psi}^{2}+R^{2} \theta_{\psi}^{2}\right)}\right] \partial_{\beta} \psi
$$

(a função $\psi$ como índice denota diferenciação com respeito a esta variável).

Tal teorema advem diretamente da solução de (2.28) em $\phi$. Com este teorema enunciado, partiremos a seguir para a obtenção de métricas com campos magnéticos, a partir de Universos de vácuo.

\subsection{Campos Magnéticos: Os Universos de Mel- vin e Ernst}

Tomemos agora uma métrica no seguinte formato,

$$
d s^{2}=f^{-1}\left[e^{2 \gamma}\left(d z^{2}+d \rho^{2}\right)+\rho^{2} d \phi^{2}\right]-f(d t-\omega d \phi)^{2}
$$

As equações de Einstein para as grandezas $f$ e $\omega$ podem ser deduzidas a partir de um lagrangeano [16],

$$
2 £=\rho f^{-2} \nabla f . \nabla f+\rho^{-1} f^{2} \nabla \omega . \nabla \omega
$$

(sendo o produto escalar em 4 dimensões) através de equações de EulerLagrange, e são escritas como

$$
\begin{aligned}
f \nabla^{2} f & =\nabla f \cdot \nabla f-\rho^{-2} f^{4} \nabla \omega \cdot \nabla \omega, \\
\nabla \cdot\left(\rho^{-2} f^{2} \nabla \omega\right) & =0 .
\end{aligned}
$$


Com alguma álgebra chegaremos a um potencial $\varepsilon$ cuja equação sintetiza (2.35-2.36). Tomemos uma função $\phi$ que não depende do ângulo azimutal $\hat{\phi}$. Então, vale a identidade $\nabla \cdot\left(\rho^{-1} \hat{\phi} \times \nabla \phi\right)=0$. Levando-se em conta (2.36) no formato $\nabla \cdot\left(\rho^{-1} \rho^{-1} f^{2} \nabla \omega\right)=0$, temos

$$
\rho^{-1} f^{2} \nabla \omega=\hat{\phi} \times \nabla \phi
$$

que relaciona nossa função com o coeficiente $\omega$. Multiplicando por $\hat{\phi}$, obtemos $f^{-2} \nabla \phi=\rho^{-1} \hat{\phi} \times \nabla \omega$, e imediatamente

$$
\nabla \cdot\left(f^{-2} \nabla \phi\right)=0
$$

Agora, levando em consideração $(\hat{\phi} \times \nabla \omega)^{2}=(\nabla \omega)^{2}$, podemos lançar mão do potencial $\varepsilon$, definido como

$$
\varepsilon=f+i \phi
$$

com o que as equações de Einstein podem ser resumidas a

$$
\left(\varepsilon+\varepsilon^{*}\right) \nabla^{2} \varepsilon=2 \nabla \varepsilon \cdot \nabla \varepsilon
$$

Um procedimento semelhante é aplicado ao incluirmos um potencial $A_{\mu}=$ $\left(A_{t}, 0,0, A_{\phi}\right)$ no invariante $(2.34)$ a partir do qual escrevemos as equações de Einstein [17]. Neste caso, devemos adicionar ao lagrangeano em (2.35 a parte correspondente ao potencial vetor,

$$
\tilde{£}=4 \rho f^{-1} A_{t}\left(\nabla A_{t}\right)^{2}-4 \rho^{-1} f\left(\nabla A_{\phi}-\omega \nabla A_{t}\right)^{2},
$$

que representa a mesma solução das equações de Einstein, mas agora com um tensor energia-momento não nulo (potencial vetor como definido acima).

Levando-se em conta uma outra função definida por $\hat{\phi} \times \Sigma=\rho^{-1} f\left(\nabla A_{\phi}-\right.$ $\left.\omega \nabla A_{t}\right)$, e o novo potencial $\Phi=A_{t}+i \Sigma$, podemos redefinir $\varepsilon$ por $\varepsilon=(f-$ $\left.|\Phi|^{2}\right)+i \phi$, com o que obteremos ao invés de uma equação de Einstein para 
um único potencial $\varepsilon$ duas equações dadas por

$$
\begin{aligned}
\left(\varepsilon+\varepsilon^{*}+|\Phi|^{2}\right) \nabla^{2} \varepsilon & =2\left(\nabla \varepsilon+2 \Phi^{*} \nabla \Phi\right) \cdot \nabla \varepsilon \\
\left(\varepsilon+\varepsilon^{*}+|\Phi|^{2}\right) \nabla^{2} \Phi & =2\left(\nabla \varepsilon+2 \Phi^{*} \nabla \Phi\right) \cdot \nabla \Phi
\end{aligned}
$$

sendo $\Phi$ um potencial adicional advindo da inclusão de $A_{\mu}$ no tensor energiamomento (ou equivalentemente no lagrangeano).

Podemos usar agora estes dois potenciais para obter, usando uma transformação de Harrison, como definido na seção anterior, a partir de uma métrica conhecida, uma outra solução com campos eletromagnéticos. Tomemos por exemplo, um elemento de linha similar ao usado nas equações (2.42) enunciadas acima sobre transformações de Harrison,

$$
d s^{2}=f^{-1}\left[-2 P^{-2} d \chi d \chi^{*}+\rho^{2} d T^{2}\right]-f\left(d \phi-\omega d T^{2}\right) .
$$

O Universo de Minkowski por exemplo, em coordenadas cilíndricas tem métrica

$$
d s^{2}=d z^{2}+d \rho^{2}-d T^{2}+\rho^{2} d \phi^{2},
$$

cuja comparação com (2.43) nos dá $f=-\rho^{2}, \omega=0, P=\rho^{-1}$ e $d \chi=$ $2^{-1 / 2}(d z+i d \rho)$. Por se tratar de uma solução de vácuo, o potencial $A_{\mu}$ é nulo, também o sendo - identicamente nula - uma das equações de Einstein pois $\Phi=0$. O potencial $\varepsilon$ de outra maneira será real e é dado necessariamente por $\varepsilon=-\rho^{2}$.

Tomando uma transformação de Harrison [14],

$$
\begin{aligned}
\bar{\varepsilon} & =\delta \varepsilon \\
\bar{\Phi} & =\delta(\Phi-B \varepsilon / 2),
\end{aligned}
$$

$\operatorname{com} \delta=\left(1+B \Phi-B^{2} \varepsilon / 4\right)^{-1}$, as variáveis $\bar{\omega}$ e $\bar{f}$ ficam

$$
\begin{aligned}
\bar{f} & =|\delta|^{2} f \\
\nabla \bar{\omega} & =|\delta|^{2} \nabla \omega+\rho f^{-1}\left(\delta^{*} \nabla \delta-\delta \nabla \delta^{*}\right) .
\end{aligned}
$$


Aqui, $B$ representa um campo magnético atuando na direção $z$ [18]. Com a métrica de Minkowski como em (2.44), as funções $\bar{f}$ e $\bar{\omega}$ com barra nos fornecem a métrica

$$
d s^{2}=\Lambda^{2}\left[d z^{2}+d \rho^{2}-d T^{2}\right]+\Lambda^{-2} \rho^{2} d \phi^{2}
$$

$\left(\Lambda=1+B^{2} r^{2} \operatorname{sen}^{2} \theta\right)$ que é uma métrica de espaço-tempo plano com campo magnético de fundo na direção $z$, obtida pela primeira vez por Melvin e Thorne [19].

Para obtermos a métrica de Ernst, tomemos o elemento de linha de Schwarzschild em coordenadas esféricas,

$$
d s^{2}=-\left(1-\frac{2 M}{r}\right) d t^{2}+\frac{d r^{2}}{1-\frac{2 M}{r}}+r^{2} d \theta^{2}+r^{2} \operatorname{sen}^{2} \theta d \phi^{2}
$$

que, quando comparado com (2.43) no dá

$$
\begin{gathered}
f=-r^{2} \operatorname{sen}^{2} \theta, \quad \omega=0, \quad \rho=\sqrt{r^{2}-2 M r} \operatorname{sen} \theta \\
P^{-1}=r^{2} \operatorname{sen} \theta, \quad d \chi=\frac{1}{\sqrt{2}}\left[\frac{d r}{\sqrt{r^{2}-2 M r}}+i d \theta\right] .
\end{gathered}
$$

Após calcularmos as funções transformadas, obtemos um elemento de linha como

$$
d s^{2}=\Lambda^{2}\left[\frac{d r^{2}}{1-2 M / r}+r^{2} d \theta^{2}-\left(1-\frac{2 M}{r}\right) d t^{2}\right]+\Lambda^{-2} r^{2} \operatorname{sen}^{2} \theta d \phi^{2} .
$$

Esta solução, obtida por Ernst em 1976, representa uma modificação da solução de Schwarzschild incluindo um campo magnético na direção $z$, e ficou conhecida na literatura como solução de Ernst. Nosso guia de estudos é o cálculo de quantidades significativas desta solução: os modos quasi-normais e a contribuição do campo escalar para a entropia do buraco negro. 


\subsection{Campos Escalares na Métrica de Ernst: os Modos Quasi-normais}

Tomemos uma métrica como em (2.53), definindo $£=B^{2} r^{2} \operatorname{sen}^{2} \theta$.

Um estudo sobre esta geometria foi feito em [20], onde se verificou a possibilidade de tal buraco negro servir como lente gravitacional. Apesar da presença de um campo magnético, o horizonte de eventos situa-se ainda em $r=2 M$ e a superfície gravitacional no horizonte também é dada por $\alpha=(4 M)^{-1}$. O objetivo, aqui, é o estudo da equação de Klein-Gordon,

$$
\square \Phi=\frac{1}{\sqrt{-g}} \partial_{\mu}\left[\sqrt{-g} g^{\mu \nu} \partial_{\nu} \Phi\right]=0,
$$

na geometria proposta. Antes de expandi-la, notamos que

$$
\begin{aligned}
\sqrt{-g} & =\Lambda^{2} r^{2} \sin \theta=\Lambda^{2} \sqrt{-g_{\text {Schwarzschild }}} \\
g^{\mu \nu} & =\operatorname{diag}\left\{\Lambda^{-2}\left[\left(1-\frac{2 M}{r}\right)^{-1},-\left(1-\frac{2 M}{r}\right),-r^{-2},-\frac{\Lambda^{4}}{r^{2} \sin ^{2} \theta}\right]\right\} .
\end{aligned}
$$

Expandindo esta equação, obtemos,

$$
g^{t t} \partial_{t t} \Phi+g^{r r} \partial_{r r} \Phi+\frac{\partial_{r}\left(g^{r r} \Lambda^{2} r^{2}\right) \partial_{r} \Phi}{\Lambda^{2} r^{2}}+P(\theta, \phi) \Phi=0
$$

$\operatorname{com} \Phi=\Phi(t, r, \theta, \phi)$. Aqui $P$ representa a parte angular do polinômio, dada por

$$
P(\theta, \phi) \Phi=\frac{g^{\theta \theta} \partial_{\theta}(\sin \theta) \partial_{\theta} \Phi}{\sin \theta}+g^{\theta \theta} \partial_{\theta \theta} \Phi+g^{\phi \phi} \partial_{\phi \phi} \Phi
$$

Ao desenvolvermos a parte angular da equação chegamos a

$$
\frac{1}{\Lambda^{2}}\left\{\left[-\frac{\cos \theta}{r^{2} \sin \theta}-\frac{\partial_{\theta}}{r^{2}}\right] \partial_{\theta} \Phi-\frac{\Lambda^{4}}{r^{2} \sin ^{2} \theta} \partial_{\phi \phi} \Phi\right\}=P \Phi
$$

Pela equação de Laplace em 4 dimensões e em coordenadas esféricas, temos 
que

$$
\frac{1}{\Lambda^{2}}\left\{\left[-\frac{\cos \theta}{r^{2} \sin \theta}-\frac{\partial_{\theta}}{r^{2}}\right] \partial_{\theta} \Phi-\frac{1}{r^{2} \sin ^{2} \theta} \partial_{\phi \phi} \Phi\right\}=\frac{l(l+1)}{\Lambda^{2}} \Phi
$$

de maneira que a idéia é somar e subtrair uma unidade na expressão com $\Lambda^{4}$. Observando que

$$
\Lambda^{4}-1=4 £+6 £^{2}+4 £^{3}+£^{4}
$$

e tomando a expressão acima em primeira ordem em $£$, conseguimos para a parte angular da equação de Klein-Gordon, a expressão

$$
\frac{1}{\Lambda^{2}}\left\{\left[-\frac{\cos \theta}{r^{2} \sin \theta}-\frac{\partial_{\theta}}{r^{2}}\right] \partial_{\theta} \Phi-\frac{\Lambda^{4}}{r^{2} \sin ^{2} \theta} \partial_{\phi \phi} \Phi\right\} \simeq \frac{\left[l(l+1)+4 m^{2} r^{2} B^{2}\right] \Phi}{r^{2} \Lambda^{2}} .
$$

A justificativa para desprezar termos em ordem maior que $B^{2} r^{2}$ é de que, os campos magnéticos, mesmo que bastante intensos, não chegam nunca próximos à ordem da grandeza da massa do buraco negro. Devemos notar ainda que na equação acima, tomamos como expressão angular para o campo,

$$
\Phi(\phi)=e^{-i m \phi},
$$

visto que $\partial_{\phi}$ é um vetor de Killing da geometria. A separação de variáveis agora é usual, seguindo o mesmo esquema de Schwarzschild, por isto ela não será feita aqui. Ao substituirmos o campo $\Phi$ por $\frac{\chi}{r}$, trocarmos a coordenada $r$, pela coordenada tartaruga $g^{r r} d r_{*}=d r$ e supondo uma dependência temporal do tipo $e^{-i \omega t}$ para o campo, chegamos à equação de onda como,

$$
\left[\frac{d^{2}}{d r_{*}^{2}}+\omega^{2}-V\left(r_{*}\right)\right] \chi\left(r_{*}\right)=0
$$

com o potencial escalar $V(r)$ dado por

$$
V\left(r_{*}\right)=g_{S c h}^{r r}\left[\frac{l(l+1)}{r^{2}}+\frac{2 M}{r^{3}}+4 B^{2} m^{2}\right]
$$


$\left[g_{S c h}^{r r}=1-\frac{2 M}{r}\right]$. Este potecial efetivo obtido para a geometria de Ernst corresponde ao potencial da geometria de Schwarzschild para a propagação de um campo escalar massivo com massa $\mu=2 m B$.

Para analisar o potencial da equação sem aproximações (e obter os modos quasi-normais), devemos usar outras condições de contorno que não as de onda plana (p. ex. C. C. de Dirichlet). Isto porque ao incluirmos todos os termos de campos magnéticos, o potencial diverge no infinito. Entretanto como citado na introdução desta seção, é suficiente para determinar os modos, estabelecer o comportamento no horizonte de eventos do buraco negro.

A questão das condições de contorno, tomadas para os modos quasinormais, de ondas planas nos infinitos espaciais, precisa neste caso ser analisada de maneira diferente. Para um buraco negro não assintoticamente plano, não é simples a definição de tais tipos de condições de contorno. Como citado no parágrafo anterior, podemos eventualmente, em espaços Anti-de Sitter, usar condições de contorno de Dirichlet. Contudo, ainda em casos não assintoticamente planos, a contribuição mais importante da perturbação da métrica é dada pela região próxima ao horizonte [21]. Além disto, a contribição da região próxima ao horizonte é determinada, principalmente, pelo formato do potencial efetivo nas proximidades de seu pico. Desta maneira, não é preciso delimitar de que maneira o campo decai quando em regiões assintóticas, para efeito do cálculo das frequências é suficiente, com o uso do método WKB, que suponhamos que assintóticamente temos uma geometria de Schwarzschild, nos atendo aqui, para a influência do campo, ao comportamento no horizonte.

Da mesma maneira como o potencial é obtido para a métrica de Ernst, podemos analisar o caso da propagação do campo escalar em um espaçotempo com campo magnético de fundo e um campo escalar dilatônico [22]. A métrica neste caso tem a forma

$d s^{2}=\Lambda^{\frac{2}{1+a^{2}}}\left[\left(1-\frac{2 M}{r}\right) d t^{2}-\left(1-\frac{2 M}{r}\right)^{-1} d r^{2}-r^{2} d \theta^{2}\right]-\frac{r^{2} \sin ^{2} \theta}{\Lambda^{\frac{2}{1+a^{2}}}} d \phi^{2}$ 
com

$$
\Lambda=1+\left(1+a^{2}\right) B^{2} r^{2} \sin ^{2} \theta
$$

sendo a um fator constante que relaciona o dilaton e o campo magnético como

$$
\frac{\phi}{\ln \Lambda}=-\frac{a}{1+a^{2}}
$$

O potencial obtido ao aplicarmos a mesma aproximação para campos magnéticos pequenos em relação à massa do buraco negro é exatamente (2.65). Isto mostra que a influência do dilaton não pode ser detectada para o termo dominante de campo magnético, visto que o fator $a$ não aparece no potecial: o campo escalar não interage com o dilaton.

Outro caso interessante a ser estudado, é o buraco negro de Ernst Ddimensional, isto é um buraco negro de Schwarzschild em D dimensões [23] imerso em um campo magnético [24]. O elemento de linha desta situação é escrito como

$d s^{2}=\Lambda^{\frac{2}{D-3}}\left[-F(r) d t^{2}+\{F(r)\}^{-1} d r^{2}+r^{2} d \theta^{2}+r^{2} \cos ^{2} \theta d \Omega_{D-4}^{2}\right]+\Lambda^{-2} r^{2} \sin ^{2} \theta d \Phi^{2}$,

com $d \Omega_{D-4}^{2}$ o elemento de linha da $(D-4)$-esfera,

$$
d \Omega_{D-4}^{2}=d \Psi_{1}^{2}+\sum_{a=1}^{D-5} d \Psi_{a+1}^{2} \prod_{b=1}^{a} \sin ^{2} \Psi_{b}
$$

em que a função $F(r)$ é o " $g^{r r}$ " da geometria D-dimensional de Schwarzschild,

$$
F(r)=1-\frac{16 \pi M(D-2)^{-1}}{r^{D-3} \Omega_{D-2}}
$$


e $\Lambda$ o termo de campo magnético,

$$
\Lambda=1+\frac{D-3}{2 D-4} B^{2} r^{2} \sin ^{2} \theta
$$

O determinante da métrica é dado por

$$
\sqrt{-g}=\Lambda^{\frac{2}{D-3}} r^{D-2} \sin \theta \cos ^{D-4} \theta \sin ^{D-5} \Psi_{1} \sin ^{D-6} \Psi_{2} \cdots \sin \Psi_{D-5}
$$

e a equação para o campo escalar torna-se

$g^{t t} \partial_{t t} \Phi+\beta \partial_{r}\left(g_{S c h}^{r r} \partial_{r} \Phi\right)+\frac{\beta(D-2) g_{S c h}^{r r} \partial_{r} \Phi}{r}+P\left(\theta, \phi, \Psi_{1}, \cdots, \Psi_{D-4}\right) \Phi=0$,

em que $\beta \equiv \Lambda^{-\frac{2}{D-3}}$, e $g_{S c h}^{\mu \nu}$ refere-se a métrica de Schwarzschild em D dimensões. Começando o estudo pela parte radial, que denominaremos " $H$ ", temos

$$
\begin{array}{r}
\Lambda^{\frac{2}{D-3}} H(r, t) \equiv g_{S c h}^{t t} \partial_{t t} \Phi+\frac{\partial_{r}\left(r^{D-2} g_{S c h}^{r r} \partial_{r} \Phi\right)}{r^{D-2}}= \\
g_{S c h}^{t t} \partial_{t t} \Phi+g_{S c h}^{r r} \partial_{r r} \Phi+\left[\partial_{r} g_{S c h}^{r r}+\frac{(D-2) g_{S c h}^{r r}}{r}\right] \partial_{r} \Phi .
\end{array}
$$

O próximo passo é decompor o campo em suas componentes para uma separação de variáveis tomando-se

$$
\Phi\left(r, t, \theta, \phi, \cdots, \Psi_{D-4}\right)=\bar{R}(r) T(t) \Theta(\theta) \varphi(\phi) \cdots \psi_{D-4}\left(\Psi_{D-4}\right) .
$$

Substituimos $\bar{R}(r) \rightarrow \frac{R(r)}{r}$ e trocamos $r$ pela sua coordenada tartaruga, $d r_{*}=$ $g_{r r} d r$. Fazendo isto, dividindo o termo $H$ pelo campo $\Phi$ e supondo $T(t)=$ $e^{-i \omega t}$ obtemos,

$$
\frac{\Lambda^{\frac{2}{D-3}} H(r, t)}{\Phi}=g_{S c h}^{t t} \omega^{2}-\frac{g_{S c h}^{t t}}{R(r)} \frac{\partial^{2} R}{\partial r_{*}^{2}}-\left(\frac{D-2}{r}\right)\left[\frac{\partial_{r} g_{S c h}^{r r}}{2}-\frac{(D-4)}{\left[g_{S c h}^{r r}\right]^{-1} 4 r}\right]
$$

Isto completa a parte radial-temporal da equação. Voltando agora para a 
parte angular,

$$
\begin{array}{r}
P\left(\theta, \phi, \Psi_{1}, \cdots, \Psi_{D-4}\right) \Phi=\frac{\partial_{\theta}\left(\sin \theta \cos ^{D-4} \theta \partial_{\theta} \Phi\right)}{\beta r^{2} \sin \theta \cos ^{D-4} \theta}+\frac{\beta^{D-3} \partial_{\phi \phi} \Phi}{r^{2} \sin ^{2} \theta} \\
+\frac{\partial_{\Psi_{1}}\left(\sin ^{D-5} \Psi_{1} \partial_{\Psi_{1}} \Phi\right)}{\beta r^{2} \cos ^{2} \theta \sin ^{D-5} \Psi_{1}}+\frac{\partial_{\Psi_{2}}\left(\sin ^{D-6} \Psi_{2} \partial_{\Psi_{2}} \Phi\right)}{\beta r^{2} \cos ^{2} \theta \sin ^{2} \Psi_{1} \sin ^{D-6} \Psi_{2}} \\
+\cdots+\frac{\partial_{\Psi_{D-5}}\left(\sin \Psi_{D-5} \partial_{\Psi_{D-5}} \Phi\right)}{\beta r^{2} \cos ^{2} \theta \sin ^{2} \Psi_{1} \sin ^{2} \Psi_{2} \cdots \sin ^{2} \Psi_{D-6} \sin \Psi_{D-5}} \\
+\frac{\left.\partial_{\Psi_{D-4} \Psi_{D-4}} \Phi\right)}{\beta r^{2} \cos ^{2} \theta \sin ^{2} \Psi_{1} \sin ^{2} \Psi_{2} \cdots \sin ^{2} \Psi_{D-5}}
\end{array}
$$

Para o caso de Schwarzschild em D dimensões, a única diferença está no segundo termo da primeira linha em que teríamos $\beta^{-1}$ ao invés de $\beta^{D-3}$. Em tal situação a parte angular se resolve como

$$
\begin{array}{r}
\tilde{P}\left(\theta, \phi, \Psi_{1}, \cdots, \Psi_{D-4}\right) \Phi=\frac{\partial_{\theta}\left(\sin \theta \cos ^{D-4} \theta \partial_{\theta} \Phi\right)}{\beta r^{2} \sin \theta \cos ^{D-4} \theta}+\frac{\partial_{\phi \phi} \Phi}{\beta r^{2} \sin ^{2} \theta} \\
+\frac{\partial_{\Psi_{1}}\left(\sin ^{D-5} \Psi_{1} \partial_{\Psi_{1}} \Phi\right)}{\beta r^{2} \cos ^{2} \theta \sin ^{D-5} \Psi_{1}}+\frac{\partial_{\Psi_{2}}\left(\sin ^{D-6} \Psi_{2} \partial_{\Psi_{2}} \Phi\right)}{\beta r^{2} \cos ^{2} \theta \sin ^{2} \Psi_{1} \sin ^{D-6} \Psi_{2}} \\
+\cdots+\frac{\partial_{\Psi_{D-5}}\left(\sin \Psi_{D-5} \partial_{\Psi_{D-5}} \Phi\right)}{\beta r^{2} \cos ^{2} \theta \sin ^{2} \Psi_{1} \sin ^{2} \Psi_{2} \cdots \sin ^{2} \Psi_{D-6} \sin \Psi_{D-5}} \\
+\frac{\left.\partial_{\Psi_{D-4} \Psi_{D-4}} \Phi\right)}{\beta r^{2} \cos ^{2} \theta \sin ^{2} \Psi_{1} \sin ^{2} \Psi_{2} \cdots \sin ^{2} \Psi_{D-5}}=-\frac{l(l+D-3) \Phi}{\beta r^{2}} .
\end{array}
$$

Podemos então achar uma relação entre as duas equações, dada por

$$
\beta(P-\tilde{P})=\left(\beta^{D-2}-1\right) \frac{\partial_{\phi \phi} \Phi}{r^{2} \sin ^{2} \theta},
$$

ou seja,

$$
P\left(\theta, \phi, \Psi_{1}, \cdots, \Psi_{D-4}\right)=\left(\frac{\beta^{D-2}-1}{\beta}\right) \frac{\partial_{\phi \phi} \Phi}{r^{2} \sin ^{2} \theta}-\frac{l(l+D-3) \Phi}{\beta r^{2}} .
$$

Lembrando que $\beta^{D-2}=\Lambda^{\frac{2 D-4}{D-3}}$, e novamente tomando a aproximação de campos fracos, temos que

$$
\beta^{D-2}-1 \simeq B^{2} r^{2} \sin ^{2} \theta
$$


de maneira que a parte angular da equação se torna,

$$
\frac{P\left(\theta, \phi, \Psi_{1}, \cdots, \Psi_{D-4}\right)}{\Phi\left(t, r, \theta, \phi, \Psi_{1}, \cdots, \Psi_{D-4}\right)}=-\frac{1}{\beta}\left[m^{2} B^{2}+\frac{l(l+D-3)}{r^{2}}\right] .
$$

Juntando as equações (2.77) e (2.83), temos

$$
\begin{array}{r}
0=\beta \frac{P\left(\theta, \phi, \Psi_{1}, \cdots, \Psi_{D-4}\right)}{\Phi\left(t, r, \theta, \phi, \Psi_{1}, \cdots, \Psi_{D-4}\right)}+\frac{\Lambda^{\frac{2}{D-3}} H(r, t)}{\Phi\left(t, r, \theta, \phi, \Psi_{1}, \cdots, \Psi_{D-4}\right)}= \\
g_{S c h}^{t t} \omega^{2}-\frac{g_{S c h}^{t t}}{R(r)} \frac{\partial^{2} R}{\partial r_{*}^{2}}-\left(\frac{D-2}{r}\right)\left[\frac{\partial_{r} g_{S c h}^{r r}}{2}-\frac{(D-4)}{\left[g_{S c h}^{r r}\right]^{-1} 4 r}\right]-\left[m^{2} B^{2}+\frac{l(l+D-3)}{r^{2}}\right] .
\end{array}
$$

Finalmente, a equação de propagação do campo é a mesma que em (2.64), mas o potencial é dado neste caso por

$$
\begin{array}{r}
V(r)=F(r)\left[\frac{l(l+D-3)}{r^{2}}+\frac{d F(r)}{d r} \frac{D-2}{2 r}+B^{2} m^{2}\right. \\
\left.+\frac{F(r)(D-4)(D-2)}{4 r^{2}}\right] .
\end{array}
$$

A presença da massa do campo escalar cria um efeito de diminuir o amortecimento dos modos, isto é, diminuir a parte imaginária destes. Na tabela I, seguem os resultados numéricos obtidos para os modos.

Pelos dados da tabela, podemos notar que enquanto a parte imaginária $\operatorname{Im}(\omega)$ descresce, a parte real $\operatorname{Re}(\omega)$ cresce, quando aumentamos o campo. Isto quer dizer que o buraco negro tem um "fator de qualidade" melhor na presença de um campo magnético, visto que a razão $Q \sim \frac{\operatorname{Re}(\omega)}{\operatorname{Im}(\omega)}$ aumenta.

Nas figuras (2.3) e (2.3) podemos ver respectivamente a evolução do campo para um valor de $B$, e o fator de qualidade variando-se o valor do campo.

Finalmente, devemos notar que mesmo sendo o campo magnético um fator que torna o buraco negro um melhor "oscilador", este efeito é pequeno: o termo de massa do campo, $4 m^{2} B^{2}$ é bastante menor do que o termo centrífugo $l(l+1) r^{-2}$. 
Tabela 2.1: Modos quasi-normais para Buracos Negros de Ernst com diferentes valores de campos magnéticos $B$ e $M=1$.

\begin{tabular}{|c|c|c|}
\hline$B$ & $\ell=1, m=1$ & $\ell=2, m=1$ \\
\hline 0.005 & $0.292981-0.097633 \mathrm{i}$ & $0.484433-0.096488 \mathrm{i}$ \\
0.025 & $0.294054-0.096988 \mathrm{i}$ & $0.486804-0.095675 \mathrm{i}$ \\
0.050 & $0.297416-0.094957 \mathrm{i}$ & $0.490764-0.094312 \mathrm{i}$ \\
0.075 & $0.303040-0.091521 \mathrm{i}$ & $0.496327-0.092389 \mathrm{i}$ \\
0.100 & $0.321199-0.080040 \mathrm{i}$ & $0.496327-0.092389 \mathrm{i}$ \\
0.125 & $0.333777-0.071658 \mathrm{i}$ & $0.503512-0.089891 \mathrm{i}$ \\
0.150 & $0.348640-0.061174 \mathrm{i}$ & $0.512346-0.086795 \mathrm{i}$ \\
0.175 & $0.365606-0.048285 \mathrm{i}$ & $0.522862-0.083070 \mathrm{i}$ \\
0.200 & $0.384366-0.032754 \mathrm{i}$ & $0.535100-0.078676 \mathrm{i}$ \\
0.225 & $0.404542-0.014468 \mathrm{i}$ & $0.549107-0.073554 \mathrm{i}$ \\
0.250 & $0.483675-0.0096748 \mathrm{i}$ & $0.564937-0.067625 \mathrm{i}$ \\
\hline
\end{tabular}

Figura 2.1: Evolução da perturbação para $B=0.05, M=1, m=1, l=1$.

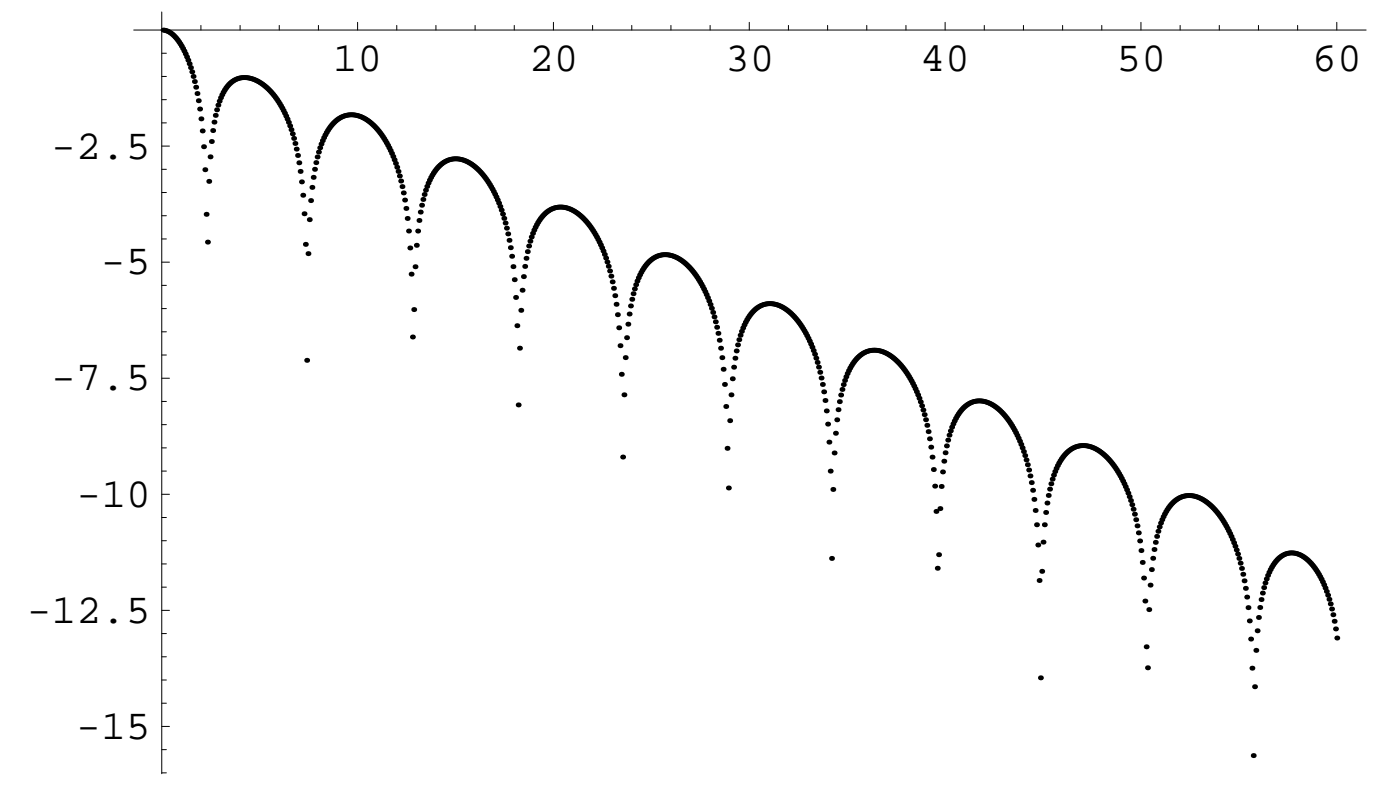


Figura 2.2: $\frac{\operatorname{Re}(\omega)}{\operatorname{Im}(\omega)}$ em função do campo magnético $B: M=1, m=1, l=1$. $\operatorname{Re} \omega / \operatorname{Im} \omega$

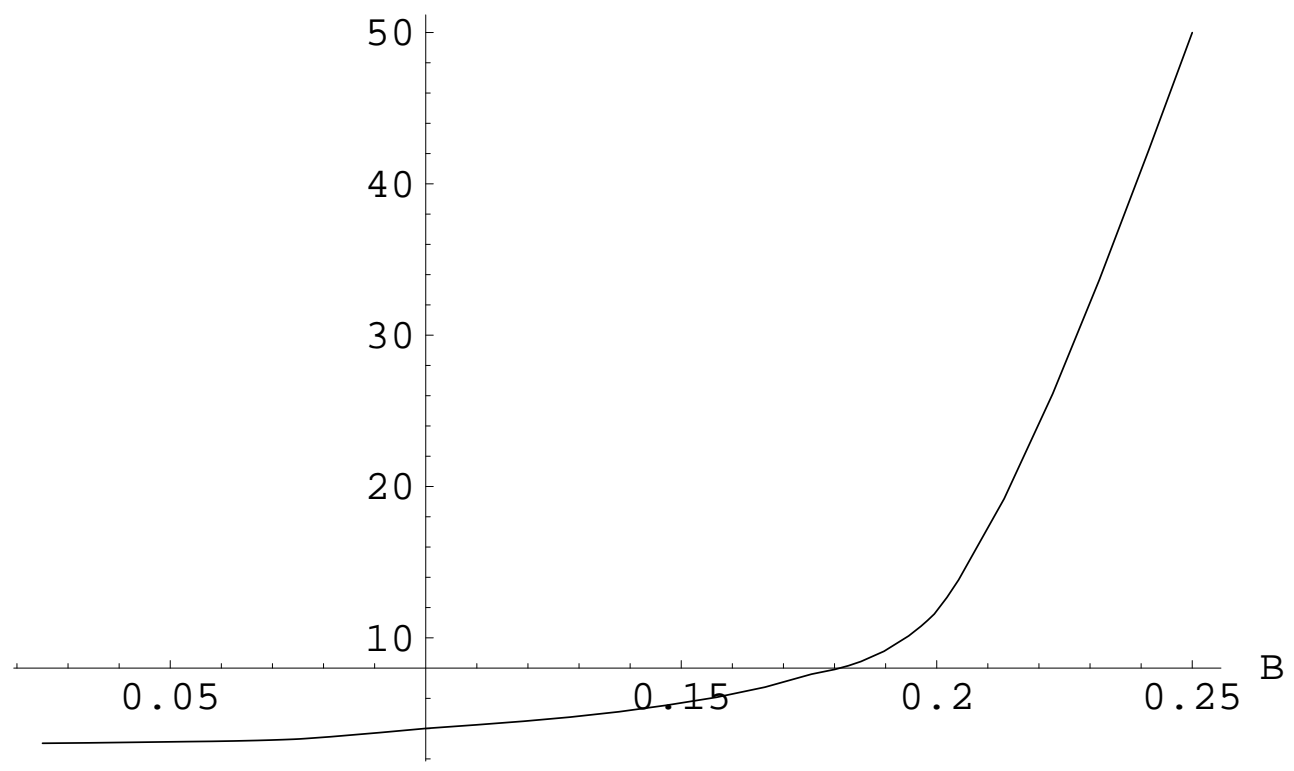

\subsection{A Entropia do Buraco Negro de Ernst: contribuição com Campo Escalar}

Um desenvolvimento bastante interessante a respeito da estrutura quântica que um buraco negro pode ter foi dado por 't Hooft em um artigo em 1985 [25]. Neste artigo, o autor calcula a entropia de um campo escalar livre em uma geometria tipo Schwarzschild e chega a mostrar que esta depende de 2 cutoffs impostos ao cálculo: um infravermelho, que não é mais do que as flutuações de vácuo do espaço-tempo, e outro ultravioleta, que representa uma característica do horizonte. De fato, 't Hooft demonstra que este último corte no ultravioleta, é fisicamente necessário: mais de $1 / 5$ da "massa total do espaço-tempo" estaria concentrada na parte de fora do buraco negro sem o corte. Não apenas isto, mas este cutoff é uma propriedade unicamente do horizonte de eventos, independendo da massa do buraco negro [26].

Nosso interesse aqui é calcular a contribuição do campo escalar para a entropia de buracos negros com um campo magnético de fundo. Para 
isto, começaremos investigando a métrica de Ernst dada por (2.53), sendo que o potencial vetor que gera o campo magnético é dado por $A_{\mu} d x^{\mu}=$ $-\frac{B r^{2} \operatorname{sen}^{2} \theta}{2 \Lambda} d \phi$.

As propriedades termodinâmicas desta solução correspondem exatamente às mesmas propriedades da solução de Schwarzschild [27]. Por exemplo, a temperatura Hawking do buraco negro é dada por $T_{H}=\left(4 \pi r_{h}\right)^{-1}$. Para calcular a contribuição do campo escalar à geometria, podemos usar, de uma maneira direta a equação de propagação do campo, e calcular o número de modos radiais, que é proporcional à função de energia livre. Isto é facilmente factível quando a equação escalar for separável em parte angular e parte radial-temporal. Entretanto, a geometria de Ernst permite tal separação apenas de uma maneira aproximada [28], e a aproximação de campo fraco não é boa o suficiente para este cálculo, visto que são necessárias muitas expansões em $B$ pequeno.

Uma maneira precisa de obter a energia livre é calcular, diretamente, via espaço de fase, o número de estados disponível. Tal cálculo é dado por

$$
\Gamma=\int d^{n} \vec{x} \int d^{n} \vec{p}
$$

em que $n$ é o número de dimensões tipo espaço. Para obter tal relação no espaço-tempo de Ernst necessitamos primeiramente das definições dos momenta, $p_{i}$. Com este intuito podemos lançar mão do método WKB, no qual utilizamos uma expressão para o campo escalar dada por

$$
\Psi(r, t, \theta, \phi) \sim e^{-i E t+i S(r, \theta, \phi)},
$$

em que as expressões para os momenta são escritas como,

$$
p_{(r, \theta, \phi)}=\partial_{(r, \theta, \phi)} S
$$

Ao usarmos a equação do campo escalar, conseguimos uma expressão para o momentum $p_{r}$ dada por

$$
p_{r}^{2}=\left(\partial_{r} S\right)^{2}=g_{r r}\left(-g^{t t} E^{2}-g^{\phi \phi} p_{\phi}^{2}-g^{\theta \theta} p_{\theta}^{2}-\mu^{2}\right),
$$


com $\mu$ representando a massa deste campo. Substituindo (2.89) na relação (2.86), obteremos

$$
\Gamma=\int d \phi d \theta d r \int d p_{\phi} d p_{\theta} \sqrt{g_{r r}\left(-g^{t t} E^{2}-g^{\phi \phi} p_{\phi}^{2}-g^{\theta \theta} p_{\theta}^{2}-\mu^{2}\right)} .
$$

Os limites de integração para os momenta são tais que exaurem, na integral, todas as possibilidades de soma sempre mantendo o integrando real e positivo. Para as coordenadas angulares, devemos somar sobre a extensão padrão, ou seja, $\phi$ entre 0 e $2 \pi$ e $\theta$ entre 0 e $\pi$. Finalmente, na coordenada radial, usamos a proposta de 't Hooft supondo dois cutoffs, um ultravioleta e outro infravermelho. Impomos assim, que o campo é zero fora dos limites estipulados,

$$
\Psi=0 \quad \text { se } \quad r \leq r_{h}+\epsilon \quad \text { ou } \quad r \geq L(\infty)
$$

Levando em consideração estas afirmações, podemos substitui-las em (2.90), com o que obtemos

$$
\begin{aligned}
\Gamma\left(E, \mu, B, r_{h}, L, \epsilon\right) & =\int_{0}^{2 \pi} d \phi \int_{0}^{\pi} d \theta \int_{r_{h}+\epsilon}^{L} d r \int_{0}^{p_{\phi}^{\text {máx }}} d p_{\phi} \int_{0}^{p_{\theta}^{\text {máx }}} d p_{\theta} * \\
& * \sqrt{g_{r r}\left(-g^{t t} E^{2}-g^{\phi \phi} p_{\phi}^{2}-g^{\theta \theta} p_{\theta}^{2}-\mu^{2}\right)}
\end{aligned}
$$

Neste caso, como afirmado acima, o valor máximo de $p_{\theta}$ é dado pelo máximo valor que esta variável pode tomar no integrando sem deixá-lo negativo ou imaginário: $p_{\theta}^{\text {máx }}=\sqrt{\frac{-g^{t t} E^{2}-g^{\phi \phi} p_{\phi}^{2}-\mu^{2}}{g^{\theta \theta}}} \equiv a$. Assim, a equação (2.92) fica sendo

$$
\begin{gathered}
\Gamma\left(E, \mu, B, r_{h}, L, \epsilon\right)=\int_{0}^{2 \pi} d \phi \int_{0}^{\pi} d \theta \int_{r_{h}+\epsilon}^{L} d r \int_{0}^{p_{\phi}^{\text {máx }}} d p_{\phi} * \\
\left.*\left[\frac{p_{\theta}}{2} \sqrt{a^{2}-p_{\theta}^{2}}+\frac{a^{2}}{2} \operatorname{arcsen} \frac{p_{\theta}}{a}\right]\right|_{0} ^{p_{\theta}^{\text {max } \equiv a}}= \\
\sqrt{g_{r r} g^{\theta \theta}} \int_{0}^{2 \pi} d \phi \int_{0}^{\pi} d \theta \int_{r_{h}+\epsilon}^{L} d r \int_{0}^{p_{\phi}^{\text {máx }}} d p_{\phi} \frac{\pi\left(-g^{t t} E^{2}-g^{\phi \phi} p_{\phi}^{2}-\mu^{2}\right)}{4 g^{\theta \theta}} .
\end{gathered}
$$


Tendo em vista que o valor máximo de $p_{\phi}$ é dado por $p_{\phi}^{\text {máx }}=\sqrt{\frac{-g^{t t} E^{2}-\mu^{2}}{g^{\phi \phi}}}$, a integral nesta variável nos dá

$\Gamma\left(E, \mu, B, r_{h}, L, \epsilon\right)=\int_{0}^{2 \pi} d \phi \int_{0}^{\pi} d \theta \int_{r_{h}+\epsilon}^{L} d r \frac{2 \pi \sqrt{g_{r r} g^{\theta \theta}} g^{\phi \phi}}{4.3 g^{\theta \theta}\left[g^{\phi \phi}\right]^{3 / 2}}\left(-g^{t t} E^{2}-\mu^{2}\right)^{3 / 2}$

Para saber a relação entre a fórmula acima e a energia livre do campo escalar, devemos investigar a função partição, que é escrita como

$$
Z=e^{-\beta F}=\sum_{N} e^{-\beta E_{N}}
$$

em que $E_{N}$ e a energia de cada estado quântico a ser somado. Para bósons, temos

$$
\sum_{n=0}^{\infty} e^{-\beta_{n} E}=\frac{1}{1-e^{-\beta E}}
$$

de maneira que a função de energia livre se torna

$$
\begin{array}{r}
F=\frac{1}{\beta} \sum_{n_{r}} \ln \left(1-e^{-\beta E}\right) \simeq \frac{1}{\beta} \int d n_{r} \ln \left(1-e^{-\beta E}\right)= \\
\frac{1}{\beta} \int\left\{d\left[n_{r} \ln \left(1-e^{-\beta E}\right)\right]-n_{r} d\left[\ln \left(1-e^{-\beta E}\right)\right]\right\}=-\int \frac{n_{r}}{e^{\beta E}-1} d E
\end{array}
$$

Na última integral, salientamos que $\int d\left[n_{r} \ln \left(1-e^{-\beta E}\right)\right]=0$, visto que para os limites $n_{r}=0$ e $E \rightarrow \infty$ tal contribuição se anula. Aqui $n_{r}$ representa o número de modos radiais, que é o mesmo que $\Gamma$ exceto por uma constante multiplicativa, $\Gamma=\pi^{3} n_{r}$. Desta maneira, a energia livre do campo escalar fica

$$
F=-\frac{1}{6 \pi^{2}} \int_{r_{h}+\epsilon}^{L} d r \int_{0}^{\pi} d \theta \int_{0}^{2 \pi} d \phi \int_{\mu \sqrt{-g_{t t}}}^{\infty} \frac{\sqrt{-g}\left(-g_{t t}^{-1} E^{2}-\mu^{2}\right)^{3 / 2}}{\sqrt{-g_{t t}}\left(e^{\beta E}-1\right)} d E
$$

No regime de aproximação em que a massa do campo escalar é pequena e o cutoff $L$ é grande, expressos por $\mu^{2} \ll \frac{r_{h}}{\epsilon \beta^{2}}$ e $L \gg r_{h}$, podemos escrever (2.98) 
como

$$
F=-\frac{1}{6 \pi^{2}} \int_{r_{h}+\epsilon}^{L} d r \int_{0}^{\pi} d \theta \int_{0}^{2 \pi} d \phi \int_{0}^{\infty} \frac{\sqrt{-g} g_{t t}^{-2} E^{3}}{\left(e^{\beta E}-1\right)} d E .
$$

A relação entre a energia livre e a entropia do campo escalar é dada por $S=$ $\beta^{2} \partial_{\beta} F$, de maneira que, ao levarmos em conta a integral $\int_{0}^{\infty} \frac{E^{3}}{e^{\beta E}-1} d E=\frac{\pi^{4}}{15 \beta^{4}}$, obtemos para a entropia do campo escalar a relação

$$
S=\frac{1}{1440 \pi r_{h}^{3}} \int d^{3} \vec{x} g_{t t}^{-2} \sqrt{-g}=\frac{1}{720 r_{h}^{3}} \int_{r_{h}+\epsilon}^{L} d r \int_{0}^{\pi} d \theta g_{t t}^{-2} \sqrt{-g}
$$

A primeira parte da relação acima é bastante geral: de fato podemos calcular a contribuição da entropia do campo escalar para uma grande classe de espaços-tempo em 4 dimensões, pelo método WKB, usando esta relação. Basta para isto que a geometria tenha elemento de linha diagonal, e que seja estacionária (o que advém da restrição em (2.87)). Neste caso, fica claro a necessidade do cutoff no ultravioleta: $g_{t t}^{-(n>1)}$ é uma função que diverge conforme nos aproximamos do horizonte de eventos.

Estamos interessados em calcular a contribuição do campo magnético na entropia do campo escalar. Procedemos então usando a relação acima com a métrica de Ernst dada por (2.53). Após a substituição dos coeficientes da métrica, resolvendo as integrais angulares, obtemos

$$
S=-\frac{1}{720 r_{h}^{3}} \int_{r_{h}+\epsilon}^{L} \frac{r^{4}}{\left(r-r_{h}\right)^{2}\left(1+B^{2} r^{2}\right)}\left[1+\frac{\operatorname{arctg} \sqrt{\frac{B^{2} r^{2}}{1+B^{2} r^{2}}}}{B r \sqrt{1+B^{2} r^{2}}}\right] d r .
$$

Esta integral é bastante complexa de se resolver, contudo, podemos fazer aproximações que nos forneçam o resultado aproximado: para as proximidades do horizonte de eventos, expandimos o integrando em torno de $r=r_{h}$ (o primeiro termo da série, que é a maior contribuição à integral é obtido simplesmente substituindo $r=r_{h}$ exceto na função $r-r_{h}$ ),

$$
S_{\epsilon}=\frac{\left(1+B^{2} r_{h}^{2}\right)^{-1}}{720 \epsilon}\left[r_{h}+\frac{\operatorname{arctg} \sqrt{\frac{B^{2} r_{h}^{2}}{1+B^{2} r_{h}^{2}}}}{B \sqrt{1+B^{2} r_{h}^{2}}}\right]
$$


Esta relação é válida para qualquer valor de $B$. Para compararmos este resultado com o obtido com 't Hooft, tomemos o limite em que este campo é pequeno, caso em que a relação acima pode ser escrita como

$$
S_{\epsilon} \simeq \frac{r_{h}}{360 \epsilon}\left[1-\frac{4}{3} B^{2} r_{h}^{2}+\frac{8}{5} B^{4} r_{h}^{4}-O\left(B^{6} r_{h}^{6}\right)\right]
$$

Este é exatamente o resultado para Schwarzschild quando $B=0$.

Para obtermos o limite ultravioleta, a partir de (2.101), podemos expandir em termos de $r \rightarrow \infty$. Neste caso, contudo, não há sentido em tomar a aproximação de $B \rightarrow 0$, para tentar recobrar o comportamento de Schwarzschild: há uma ambigüidade no integrando quando fazemos isto, a saber, funções do tipo $B^{2} r^{2}$. Não há sentido em expandir tais funções para $B=0$ quando $r \rightarrow \infty$. Se, entretanto, assumimos que na expansão $r \rightarrow \infty$, o comportamento de $B$ é tal que $B \rightarrow \frac{1}{r}$, recobramos o limite de Schwarzschild. Isto fica claro já pelos coeficientes da métrica de Ernst: com tal aproximação para $B$, (2.53) é exatamente a geometria de Schwarzschild.

Seguiremos com o cálculo a partir de (2.101), para a expansão no limite $r \rightarrow \infty$. Com tal artifício, desprezando termos $\frac{1}{r^{2}}$ ou maiores, obtemos como contribuição da entropia no limite infravermelho,

$$
S_{L} \simeq-\frac{1}{720 r_{h}^{3}}\left[\frac{1}{B^{2}} L+\frac{2 r_{h}}{B^{2}} \ln L+O\left(\frac{1}{L}\right)\right]
$$

Para o resultado acima, vale a afirmação de há poucos parágrafos: no limite em que $B \rightarrow \frac{1}{L}$, recuperamos o caráter de Schwarzschild. Neste sentido, o campo magnético age como um regularizador, diminuindo a divergência na contagem dos modos do espaço-tempo. Nota-se também que não faz sentido o limite $B \rightarrow 0$, pela ambigüidade de que falamos acima.

Há a possibilidade ainda de calcularmos o limite de campo magnético baixo, com outro procedimento: expandimos (2.101) nas imediações de $B \rightarrow$ 0 . Neste caso, entretanto, o nosso cutoff infravermelho deve ser tal que $B L<$ $A$, ou não faria sentido tal expansão ( $A$ um parâmetro genérico). Isto equivale à suposição de que nem o cutoff nem o campo magnético tomam valores 0 ou $\infty$. Fisicamente isto pode ser justificável para o estudo, por exemplo, 
de mini-buracos negros, com campos magnéticos unidirecionais grandes em relação à massa do buraco negro, mas pequenos em relação ao cutoff. Nesta situação, a contribuição total para a entropia do campo escalar é dada por

$$
\begin{gathered}
S_{B} \simeq-\frac{L^{3}}{1080 r_{h}^{3}}-\frac{r_{h}^{5}}{180}\left[\frac{2 L^{5}}{15}+\frac{L^{4}}{3}+\frac{2 L^{3}}{15}\right] B^{2}+O\left(L^{n} B^{4}\right)+ \\
\frac{r_{h}}{360 \epsilon}\left[1-\frac{4}{3} r_{h}^{2} B^{2}\right]+O\left(r_{h}^{4} B^{4}\right) .
\end{gathered}
$$

Isto encerra o cálculo para o buraco negro de Ernst. Apontamos que a contribuição do campo escalar que se propaga em tal geometria não pode ser colocada em termos de uma renormalização da constante de Newton, como sugeriram Susskind e Uglum [29], ou mesmo, como pode ser visualizado, de uma maneira a reinterpretar a constante newtoniana [30]. Tal fato era esperado, já que a métrica de Ernst não é assintoticamente plana, ou seja, não obtemos a gravitação newtoniana com $r \rightarrow \infty$.

A interpretação desta contribuição em termos de cutoffs ainda está em discussão.

Seguiremos na mesma linha de pensamento trabalhando agora em duas generalizações dos cálculos acima: o caso do buraco negro de Ernst com um campo dilatônico [31] e o caso em $D$ dimensões [24].

\subsubsection{O buraco negro dilatônico}

A geometria de Ernst com um campo dilatônico de fundo é dada por [22]

$$
d s^{2}=\Lambda^{\frac{2}{1+a^{2}}}\left[\left(1-\frac{r_{h}}{r}\right) d t^{2}-\left(1-\frac{r_{h}}{r}\right)^{-1} d r^{2}-r^{2} d \theta^{2}\right]-\frac{r^{2} \operatorname{sen}^{2} \theta}{\Lambda^{\frac{2}{1+a^{2}}}} d \phi^{2},
$$

em que $\Lambda=1+\left(1+a^{2}\right) B^{2} r^{2} \sin ^{2} \theta$, e o dilaton se acopla com o campo magnético pela relação

$$
-\frac{a}{1+a^{2}}=\frac{\phi}{\ln \Lambda}
$$


Podemos partir de (2.100), e desenvolver a integral com os coeficientes da métrica acima,

$$
S=\frac{1}{720 r_{h}^{3}} \int_{r_{h}+\epsilon}^{L} d r \int_{0}^{\pi} d \theta \frac{\operatorname{sen} \theta}{\left[1+\left(1+a^{2}\right) B^{2} r^{2} \operatorname{sen}^{2} \theta\right]^{\frac{2}{1+a^{2}}}} \frac{r^{4}}{\left(r-r_{h}\right)^{2}}
$$

cuja integral em $\theta$ resulta em

$$
S=\frac{1}{360 r_{h}^{3}} \int_{r_{h}+\epsilon}^{L} d r \frac{{ }_{2} F_{1}\left[\frac{1}{2}, \frac{2}{1+a^{2}}, \frac{3}{2}, \frac{\left(1+a^{2}\right) B^{2} r^{2}}{1+\left(1+a^{2}\right) B^{2} r^{2}}\right]}{\left[1+\left(1+a^{2}\right) B^{2} r^{2}\right]^{\frac{2}{1+a^{2}}}} \frac{r^{4}}{\left(r-r_{h}\right)^{2}} .
$$

Aqui novamente a integral não pode ser resolvida analiticamente, e recorremos a aproximações: expandimos em séries de Taylor para regiões nas proximidades do horizonte. A contribuição para $r$ grande não pôde ser obtida ainda, já que a expansão para $r \rightarrow \infty$ não é convergente. Para as proximidades do horizonte, temos

$$
S=\frac{r_{h}}{360 \epsilon} \frac{{ }_{2} F_{1}\left[\frac{1}{2}, \frac{2}{1+a^{2}}, \frac{3}{2}, \frac{\left(1+a^{2}\right) B^{2} r_{h}^{2}}{1+\left(1+a^{2}\right) B^{2} r_{h}^{2}}\right]}{\left[1+\left(1+a^{2}\right) B^{2} r_{h}^{2}\right]^{\frac{2}{1+a^{2}}}}
$$

Também, da mesma maneira como sugerimos no caso de Ernst, podemos obter aproximadamente a contribuição para $B$ muito pequeno. A entropia neste caso é dada por

$$
\begin{gathered}
S_{d i l}=\frac{L^{3}}{1080}-\frac{1+a}{1+a^{2}}\left[r_{h}^{2} L^{3}+\frac{r_{h} L^{4}}{2}+\frac{L^{5}}{5}\right] \frac{B^{2}}{270} \\
+\frac{r_{h}}{360 \epsilon}\left\{1+\left[\frac{2}{3}-2\left(1+a^{2}\right)\right] B^{2} r_{h}^{2}\right\} .
\end{gathered}
$$

Esta relação é bastante parecida com (2.105), e de fato, para $a=0$, ela se torna (2.105), como esperado: no limite em que o campo dilatônico é nulo, recobramos a geometria de Ernst e, neste sentido, a contribuição do campo escalar à entropia deve ser a mesma. As propriedades neste caso, são as mesmas como explicado no caso anterior. Seguiremos com a última etapa do trabalho, o campo escalar em uma geometria de Ernst em $D$ dimensões. 


\subsubsection{O caso $D$-dimensional}

Um buraco negro de Ernst em $D$ dimensões tem o elemento de linha dado por $[24]$

$$
d s^{2}=\Lambda^{\frac{2}{D-3}}\left[-f d t^{2}+f^{-2} d r^{2}+r^{2} \cos ^{2} \theta d \Omega_{D-4}^{2}+r^{2} d \theta^{2}\right]+\Lambda^{-2} r^{2} \operatorname{sen}^{2} \theta d \phi^{2}
$$

em que $\Lambda$ é o termo com campo magnético dado por

$$
\Lambda=1+\frac{D-3}{2 D-4} B^{2} r^{2} \operatorname{sen}^{2} \theta
$$

$d \Omega_{D-4}^{2}=\sum_{j=1}^{D-4} \prod_{i=1}^{j-1} \sin ^{2} \Psi_{i} d \Psi_{j}^{2}$ é a $(D-4)$-esfera e $f$ a função de Schwarzschild em $D$ dimensões escrita como $f=1-\left(\frac{r_{h}}{r}\right)^{D-3}$, com $r_{h}$ o raio do horizonte de eventos definido por $r_{h}=\left[\frac{16 \pi M}{(D-2) \Omega_{D-2}}\right]^{\frac{1}{D-3}}$. As propriedades termodinâmicas deste buraco negro são as mesmas daquelas do buraco negro de Schwarzschild em $D$ dimensões.

Estamos procurando, de início, como o fizemos na seção anterior, a energia livre de Helmholtz, com o que poderemos calcular a entropia do campo escalar. Com tal objetivo, partimos novamente de (2.86), mas generalizando tal expressão para $D$ dimensões. Temos

$$
\Gamma=\int \prod_{i=1}^{D-3} d \Psi_{i} d \phi d r d p_{\Psi_{i}} d p_{\phi} \sqrt{g_{r r}\left(-g^{t t} E^{2}-g^{\phi \phi} p_{\phi}^{2}-\sum_{j=1}^{D-3} g^{\Psi_{i} \Psi_{i}} p_{\Psi_{i}}^{2}\right)}
$$

Para obter uma fórmula geral a partir da integral no espaço dos momenta, partiremos de (2.94) como início, primeiro tratando o caso 5-dimensional depois o fazendo para os demais, até 8 dimensões. Tal repetição embora pareça desnecessária, não o é de fato, pois devemos achar o termo geral de duas séries alternantes, para o que o número mínimo de termos é 4, como 
ficará claro mais adiante. Desta maneira tomemos

$\Gamma_{5}=\int d^{4} \vec{r} \int_{0}^{p_{\Psi_{1}}^{\text {máx }}} d p_{\Psi_{1}}\left[\frac{\pi}{2}\right]\left[\frac{1}{2}\right]\left[1-\frac{1}{3}\right] \sqrt{\frac{g}{g_{t t} g_{\Psi_{1} \Psi_{1}}}}\left(-g^{t t} E^{2}-g^{\Psi_{1} \Psi_{1}} p_{\Psi_{1}}^{2}-\mu^{2}\right)^{3 / 2}$

que integrada na última coordenada de momentum fica

$$
\Gamma_{5}=\int d^{4} \vec{r}\left[\frac{\pi}{2}\right]\left[\frac{1}{2}\right]\left[1-\frac{1}{3}\right]\left[\frac{\pi}{2}\right]\left[\frac{3}{8}\right] \sqrt{\frac{g}{g_{t t}}}\left(-g^{t t} E^{2}-\mu^{2}\right)^{2} .
$$

Chamaremos $\alpha_{5}$ os coeficientes numéricos em frente a integral, ou seja,

$\alpha_{5}=\left[\frac{\pi}{2}\right]\left[\frac{1}{2}\right]\left[1-\frac{1}{3}\right]\left[\frac{\pi}{2}\right]\left[\frac{3}{8}\right]=\frac{\pi^{2}}{32}$. Seguimos usando este cálculo acima, para o caso 6-dimensional, que é semelhante. A função de estados em 6 dimensões, a partir da fórmula citada é dada por

$$
\Gamma_{6}=\int d^{5} \vec{r} \int_{0}^{p_{\Psi_{2}}^{\text {máx }}} d p_{\Psi_{2}} \alpha_{5} \sqrt{\frac{g}{g_{t t} g^{\Psi_{2} \Psi_{2}}}}\left(-g^{t t} E^{2}-g^{\Psi_{2} \Psi_{2}} p_{\Psi_{2}}^{2}-\mu^{2}\right)^{2},
$$

cuja integração nos fornece

$$
\Gamma_{6}=\int d^{5} \vec{r} \alpha_{5}\left[1-\frac{2}{3}+\frac{1}{5}\right] \sqrt{\frac{g}{g_{t t}}}\left(-g^{t t} E^{2}-\mu^{2}\right)^{5 / 2} .
$$

Novamente, chamemos $\alpha_{6}=\left[\frac{\pi}{2}\right]\left[\frac{1}{2}\right]\left[1-\frac{1}{3}\right]\left[\frac{\pi}{2}\right]\left[\frac{3}{8}\right]\left[1-\frac{2}{3}+\frac{1}{5}\right]=\frac{\pi^{2}}{60}$.

Em 7 dimensões temos

$$
\Gamma_{7}=\int d^{6} \vec{r} \int_{0}^{p_{\Psi_{3}}^{\text {máx }}} d p_{\Psi_{3}} \alpha_{6} \sqrt{\frac{g}{g_{t t} g^{\Psi_{3} \Psi_{3}}}}\left(-g^{t t} E^{2}-g^{\Psi_{3} \Psi_{3}} p_{\Psi_{3}}^{2}-\mu^{2}\right)^{5 / 2},
$$

$\mathrm{Ou}$,

$$
\Gamma_{7}=\int d^{6} \vec{r} \alpha_{6}\left[\frac{\pi}{2}\right]\left[\frac{15}{48}\right] \sqrt{\frac{g}{g_{t t}}}\left(-g^{t t} E^{2}-\mu^{2}\right)^{3},
$$

em que redefinimos $\alpha_{7}=\alpha_{6}\left[\frac{\pi}{2}\right]\left[\frac{15}{48}\right]$. Finalmente, em 8 dimensões, a função 
de estados fica

$$
\Gamma_{8}=\int d^{7} \vec{r} \int_{0}^{p_{\Psi_{4}}^{\text {máx }}} d p_{\Psi_{4}} \alpha_{7} \sqrt{\frac{g}{g_{t t} g^{\Psi_{4} \Psi_{4}}}}\left(-g^{t t} E^{2}-g^{\Psi_{4} \Psi_{4}} p_{\Psi_{4}}^{2}-\mu^{2}\right)^{3}
$$

ou seja,

$$
\Gamma_{8}=\int d^{7} \vec{r} \alpha_{7}\left[1-\frac{3}{3}+\frac{3}{5}-\frac{1}{7}\right] \sqrt{\frac{g}{g_{t t}}}\left(-g^{t t} E^{2}-\mu^{2}\right)^{7 / 2}
$$

Tendo formulado a função $\Gamma$ para 4 dimensões diferentes, estamos agora em posição de obter uma fórmula geral para ela. Pelos desenvolvimentos acima, podemos demonstrar que

$$
\Gamma(D)=\int d^{D-1} \vec{r} \alpha_{D} \sqrt{\frac{g}{g_{t t}}}\left(-g^{t t} E^{2}-\mu^{2}\right)^{\frac{D-1}{2}},
$$

em que $\alpha_{D}$ é uma constante de integrações sucessivas, que pode ser representada pelas duas séries entre chaves,

$\alpha_{D}=\left\{\left[\frac{\pi}{2}\right]^{f(D)} \prod_{a=1}^{D-2}\left[\frac{\left(2 g_{a}-1\right) ! !}{\left(2 g_{a}\right) ! !}\right]^{s(a)}\right\}\left\{\prod_{b=0}^{D-1}\left[\sum_{c=1}^{b+1} \frac{(i)^{c-1}+(-i)^{c-1}}{2 c}\left(\begin{array}{l}f(c) \\ k(b)\end{array}\right)\right]^{s(b)}\right\}$

com

$$
\begin{aligned}
f(y) & =\frac{y-2+|\operatorname{sen}(y \pi / 2)|}{2}, \\
g_{a} & =\frac{a+|\operatorname{sen}(a \pi / 2)|}{2}, \\
s(x) & =\frac{1+(-1)^{x+1}}{2} \\
k(b) & =\frac{b-1+|\cos (b \pi / 2)|}{2} .
\end{aligned}
$$

Esta fórmula é válida para um grande número de espaços-tempo em $D$ dimensões: todos para os quais a equação de Klein-Gordon, bem como o método WKB é válido, ou seja, para as geometrias estacionárias e com 
Tabela 2.2: Valores de $\alpha_{D}$ em função de $D$.

\begin{tabular}{|c|c|c|c|c|c|c|c|c|}
\hline$D$ & 4 & 5 & 6 & 7 & 8 & 9 & 10 & 11 \\
\hline$\alpha_{D}$ & $\frac{\pi}{6}$ & $\frac{\pi^{2}}{32}$ & $\frac{\pi^{2}}{60}$ & $\frac{\pi^{3}}{384}$ & $\frac{\pi^{3}}{840}$ & $\frac{\pi^{4}}{6144}$ & $\frac{\pi^{4}}{15120}$ & $\frac{\pi^{5}}{122880}$ \\
\hline
\end{tabular}

métrica diagonal.

Os valores da constante $\alpha$ em função da dimensão do espaço-tempo podem ser vistos na tabela 2.1. Levando-se em consideração o limite em que a massa do campo é pequena, a energia livre de Helmholtz e a entropia podem ser escritas como

$$
\begin{array}{r}
F=\alpha_{D} \delta_{D} \int d^{D-1} \vec{r} \sqrt{g} g_{t t}^{-D / 2} \\
S=-\beta \alpha_{D} \overline{\delta_{D}} \int d^{D-1} \vec{r} \sqrt{g} g_{t t}^{-D / 2},
\end{array}
$$

em que definimos

$$
\delta_{D}=\int_{0}^{\infty} \frac{E^{D-1}}{e^{\beta E}-1} d E, \quad \overline{\delta_{D}}=D \delta_{D} .
$$

As equações (2.129) e (2.130) são novamente bastante gerais: para os mesmos tipo de espaços-tempo em $D$ dimensões como citados acima, podemos utilizálas para obter a energia livre de Helmholtz ou a Entropia do campo escalar.

Dando prosseguimento ao cálculo, voltamo-nos agora para o caso em 5 dimensões. A entropia pode, de acordo com as relações acima ser escrita como

$$
\begin{array}{r}
S_{5}=-5 \beta \alpha_{5} \delta_{5} \int_{0}^{\pi} d \phi \int_{0}^{2 \pi} d \Psi_{1} \int_{r_{h}+\epsilon}^{L} \int_{0}^{\pi / 2} \frac{\sin \theta \cos \theta}{\left[1+a^{2} \sin ^{2} \theta\right]^{3 / 2}} d \theta * \\
* \frac{r^{8}}{\left(r^{2}-r_{h}^{2}\right)^{5 / 2}} d r
\end{array}
$$

com $a=B r / \sqrt{3}$. As integrais em $\Psi_{1}$ e $\Phi$ são triviais e a integral em $\theta$ pode ser resolvida com uma substituição simples, $x=\operatorname{sen} \theta$. Neste caso, obtemos

$$
S_{5}=\frac{45 \zeta(5)}{32 r_{h}^{4}} \int_{r_{h}+\epsilon}^{L} \frac{r^{6}}{B^{2} \sqrt{\left(r^{2}-r_{h}^{2}\right)^{5}}}\left[1-\frac{1}{\sqrt{1+B^{2} r^{2} / 3}}\right] d r .
$$


Novamente podemos fazer as expansões para as regiões próxima ao horizonte e assintótica. Com isto (2.133) fica

$$
\begin{array}{r}
S_{5}=\frac{45 \zeta(5)}{32 r_{h} B^{2}}\left\{\frac{r_{h}^{2}}{3\left(2 r_{h} \epsilon\right)^{3 / 2}}\left[1-\frac{1}{\left(1+B^{2} r_{h}^{2} / 3\right)^{1 / 2}}\right]+\right. \\
\left.\frac{1}{\left.3\left(2 r_{h} \epsilon\right)^{1 / 2}\right)}\left[\frac{5}{2}-\frac{1-14\left(1+B^{2} r_{h}^{2} / 3\right)^{-1}}{6\left(1+B^{2} r_{h}^{2} / 3\right)^{1 / 2}}\right]\right\}- \\
\frac{45 \zeta(5)}{32 B^{2} r_{h}^{4}}\left[\frac{L^{2}}{2}+\frac{B r_{h}^{2}\left(21+5 B^{2} r_{h}^{2}\right)}{\sqrt{3}\left(3+B^{2} r_{h}^{2}\right)^{2}} L\right] .
\end{array}
$$

Para 6 dimensões, a expressão de que devemos partir é,

$$
\begin{aligned}
S_{6}= & -\frac{4 \pi^{3}}{315\left(4 r_{h}\right)^{5}} \int_{0}^{\pi} d \phi \int_{0}^{\pi} \operatorname{sen} \Psi_{1} d \Psi_{1} \int_{0}^{2 \pi} d \Psi_{2} * \\
& * \int_{r_{h}+\epsilon}^{L} \int_{0}^{\pi / 2} \frac{\operatorname{sen} \theta \cos ^{2} \theta}{\left[1+\alpha^{2} \operatorname{sen}^{2} \theta\right]^{7 / 3}} d \theta \frac{r^{13}}{\left(r^{3}-r_{h}^{3}\right)^{3}} d r
\end{aligned}
$$

com $\alpha=\frac{3 B^{2} r^{2}}{8}$. Integrando nas partes angulares obtemos

$$
\begin{aligned}
S_{6}=- & \frac{1}{105\left(4 r_{h}\right)^{5}} \int_{r_{h}+\epsilon}^{L} \frac{1}{\alpha^{2}\left(1+\alpha^{2}\right)} \frac{r^{13}}{\left(r^{3}-r_{h}^{3}\right)^{3}} * \\
& *\left\{2 \alpha^{2}-1+\frac{{ }_{2} F_{1}\left[\frac{1}{2}, \frac{1}{3}, \frac{3}{2}, \frac{\alpha^{2}}{1+\alpha^{2}}\right]}{\left(1+\alpha^{2}\right)^{2 / 3}}\right\} d r
\end{aligned}
$$

e com os mesmos artifícios usados até aqui, a expressão acima tem as maiores contribuições dada pela expressão

$$
\begin{array}{r}
S_{6}=-\frac{27 \pi^{5}}{2240 r_{h}^{5}}\left\{\frac{L^{3}}{B^{2}}-\frac{12 L}{B^{4}}+\frac{8 \pi^{1 / 3} 3^{1 / 6}}{\Gamma(1 / 3) \Gamma(7 / 6) B^{14 / 3}} L^{1 / 3}+\frac{9 r_{h}^{3}}{B^{2}} \ln L+\right. \\
\left.r_{h}^{3}\left[\frac{\left(9 B^{2} r_{h}^{2}-12\right)}{\left[B^{2} r_{h}^{2}\left(8+3 B^{2} r_{h}^{2}\right)\right]}+\frac{24{ }_{2} F_{1}\left[\frac{1}{2}, \frac{1}{3}, \frac{3}{2}, \frac{3 B^{2} r_{h}^{2}}{8+3 B^{2} r_{h}^{2}}\right]}{\left[B^{2} r_{h}^{2}\left(8+3 B^{2} r_{h}^{2}\right)^{4 / 3}\right]}\right]\left[\frac{r_{h}^{2}}{48 \epsilon^{2}}+\frac{5 r_{h}}{54 \epsilon}-\frac{5 \ln \epsilon}{27}\right]\right\} .
\end{array}
$$

Finalmente, calculamos a contribuição do campo para um caso em 10 di- 
mensões. A integral de partida é

$$
\begin{aligned}
S_{10}= & \frac{80 * 7^{9} \pi^{4} \sigma(9)}{3\left(4 \pi r_{h}\right)^{9}} \int_{0}^{\pi} d \phi \int_{0}^{\pi} \operatorname{sen}^{5} \Psi_{1} d \Psi_{1} \int_{0}^{\pi} \operatorname{sen}^{4} \Psi_{2} d \Psi_{2} * \\
& * \int_{0}^{\pi} \operatorname{sen}^{3} \Psi_{3} d \Psi_{3} \int_{0}^{\pi} \operatorname{sen}^{2} \Psi_{4} d \Psi_{4} \int_{0}^{\pi} \operatorname{sen} \Psi_{5} d \Psi_{5} * \\
& * \int_{0}^{2 \pi} d \Psi_{6} \int_{r_{h}+\epsilon}^{L} \int_{0}^{\pi / 2} \frac{\operatorname{sen} \theta \cos ^{6} \theta}{\left[1+\chi^{2} \operatorname{sen}^{2} \theta\right]^{8 / 7}} d \theta \frac{r^{43}}{\left(r^{7}-r_{h}^{7}\right)^{5}} d r
\end{aligned}
$$

com $\chi^{2}=\frac{7 B^{2} r^{2}}{16}$. Novamente, a parte angular nos fornece

$$
\begin{gathered}
S_{10} \sim \frac{7^{9} \pi^{9}}{2^{11} * 3^{5} * 11^{2} * 19 r_{h}^{9}} \int_{r_{h}+\epsilon}^{\infty} \frac{4}{\sqrt{7} B \chi^{6}} d \chi * \\
*\left\{-735-1400 \chi^{2}-627 \chi^{4}+735\left(1+\chi^{2}\right)_{2}^{13 / 7} F_{1}\left[\frac{1}{2}, \frac{1}{7}, \frac{3}{2}, \frac{\chi^{2}}{1+\chi^{2}}\right]\right\},
\end{gathered}
$$

que, com as expansões sugeridas acima resulta em

$$
\begin{array}{r}
S_{10} \sim \frac{3,8}{r_{h}^{9}}\left\{\frac{1,14}{B^{2}} L^{7}+\frac{24,1 B^{-16 / 7}}{\Gamma(1 / 7) \Gamma(19 / 14)} L^{47 / 7}+\frac{3,1 B^{-4}}{\Gamma(1 / 7) \Gamma(13 / 7)} L^{5}\right. \\
+T\left(L^{33 / 7}, L^{3}, L^{19 / 7}, \cdots\right)+10^{-3} r_{h}^{3}\left[\frac{r_{h}^{4}}{\epsilon^{4}}-\frac{8 r_{h}^{3}}{\epsilon^{3}}+\frac{81 r_{h}^{2}}{\epsilon^{2}}-\frac{1080 r_{h}}{\epsilon}\right] \\
\left.\left.+\frac{2,31 B^{2} r_{h}^{2}+0,452 B^{4} r_{h}^{4}+2,77\left\{1+\left(1+B^{2} r_{h}^{2}\right)_{2}^{2} F_{1}\left[\frac{1}{2}, \frac{1}{7}, \frac{3}{2}, \frac{7 B^{2} r_{h}^{2}}{16+7 B^{2} r_{h}^{2}}\right]\right\}}{B^{6}}\right]\right\} .
\end{array}
$$

O comportamento das divergências aqui é bastante geral: tanto no caso do buraco negro dilatônico quanto nos de Ernst, as divergências da região ultravioleta dependem do fator de cutoff como

$$
S \sim \frac{1}{\epsilon^{\frac{D-2}{2}}}
$$

Neste sentido, temos os mesmos resultados como os de Schwarzschild. Entretanto, embora não diretamente no fator de cutoff $\epsilon$, a entropia tem uma 
contribuição não-trivial do campo magnético, como pode ser visto em (2.103), o mesmo sendo verdade para os casos dilatônico e D-dimensional.

Para a região do infravermelho, tudo o que se pode supor, é que recuperamos o comportamento de Schwarzschild com a condição de que $B \rightarrow \frac{1}{L}$, pois como afirmamos acima, o limite $B \rightarrow 0$ é ambíguo, uma vez que tomamos a expansão $r \rightarrow \infty$ para resolver as integrais.

A maior divergência tem a forma

$$
S_{L} \sim \frac{L^{D-3}}{B^{2}}
$$

e as demais divergências como

$$
S_{L}(\operatorname{div}) \sim \frac{L^{a}}{B^{b}}
$$

com $a+b=D-1$, em que $b \geq 2$, exceto por divergências de escala logarítmica. Neste sentido, podemos afirmar que o campo magnético atua como um campo regularizador da divergência na região do infravermelho, como também o afirmamos para o caso 4-dimensional, pois a divergência diminui de um fator $L^{D-1}$, como seria o caso dos espaços-tempo de Minkowski ou de Schwarzschild, para $L^{D-3}$. 


\section{Capítulo 3}

\section{Modos Quasi-Normais e Termodinâmica em Buracos Negros}

Um interessante ramo do estudo de perturbações em buracos negros, é a possível conexão entre a perturbação de uma dada geometria - ou ainda a propagação de um campo teste - e as propriedades termodinâmicas desta geometria [35]. Neste âmbito, uma questão de importância relevante a ser colocada é se a área do horizonte de eventos possui um quantum determinado, e ainda se esta determinação está de alguma maneira associada ao espectro perturbativo das equações de propagação de campos teste na geometria.

A questão acerca de um determinado quantum para a área do horizonte de eventos tem seu trabalho pioneiro na década de 70, quando Bekenstein observa que a área de buracos negros não extremos se comporta como um invariante adiabático. Tendo em vista o princípio de Ehrenfest isto corresponde à discretização da área do buraco negro.

Embora alguns anos antes, Christodoulou [36] tivesse demonstrado que a assimilação de uma partícula neutra pontual pelo buraco negro era um processo reversível (e portanto com uma possível variação para área arbitrariamente baixa, $\delta A \rightarrow 0$ ), se levarmos em conta que a referida partícula não é pontual e tem um raio mínimo $r$ e uma massa $m$, o quantum de área será 
$\delta A=8 \pi m r$, e para uma partícula relativística $(r \geq \hbar / m), \delta A=8 \pi l_{P}^{2}$, com $l_{P}^{2}=\hbar[37]$.

Contudo, um outro limite é obtido se levamos em conta o buraco negro de Reissner-Nordström, com partículas carregadas se propagando ao redor de sua geometria. Supondo que estas partículas tenham raio, massa e carga definidos, neste caso, o quantum de área na geometria de Reissner-Nordström seria dado por $\delta A=4 l_{P}^{2}[38]$.

Nos dois casos (partículas carregadas ou sem carga), há um processo impedindo que o quantum de área seja arbitrariamente pequeno. Para o processo de captura de uma partícula sem carga em um buraco negro de Schwarzchild, devemos considerar que a partícula tem um raio mínimo em conformidade com o princípio da incerteza de Heisenberg, para que haja um quantum de área fundamental. No segundo processo, para assegurar a existência de um quantum mínimo de área, é preciso levar em consideração o processo de "emissão de carga tipo Schwinger". O campo elétrico crítico para a produção de pares deve ser maior do que o campo nas imediações do horizonte, levando a $\delta A_{\min }=4 l_{P}^{2}$.

\subsection{A Conjectura Hod}

Tendo em conta estes resultados, um significativo insight foi dado há pouco mais de uma década quando Hod publicou um trabalho relacionando os modos quasi-normais da geometria de Schwarzschild com a termodinâmica do respectivo buraco negro. O quantum de área proposto inicialmente por Hod, baseado nos resultados descritos acima foi expresso como

$$
\delta A=k l_{P}^{2}
$$

com $k$ uma constante numérica a ser determinada.

A idéia original reside no fato singular de que o espectro quasi-normal da solução de Schwarzschild é peculiar no regime assintótico: a parte real destas frequências converge para um valor fixo, a partir de um valor de $n$ (número de sobretom) suficientemente grande. De fato, o espectro assintótico dos modos 
quasi-normais do buraco negro de Schwarzschild é dado por [34]

$$
M \omega_{n}=M\left(\omega_{n}^{\{R\}}+\omega_{n}^{\{I\}}\right)=\frac{\ln 3}{8 \pi}-\frac{i}{4}(n+1 / 2)+O\left(\sqrt{n^{-1}}\right) .
$$

A correspondência proposta, nomeada posterirmente como conjectura de Hod, é resultante da idéia de que $\omega_{n}^{\{R\}}$ poderia representar o quantum de energia do buraco negro para transições de fase em altas energias, ou, em outras palavras, de um buraco negro não-excitado para um buraco negro que emitiria em altos valores de $n$. Desta maneira, poderíamos ter uma quantização da área do buraco negro, como se segue. A área do horizonte de eventos, $A=16 \pi M^{2}$ tem um "quantum" de

$$
\delta A=32 \pi M \delta M=32 \pi M \hbar \omega_{n}^{R}=4 \ln 3 l_{P}^{2},
$$

em que $l_{P}$ é o comprimento de Planck também escrito em unidades convenientes $(G=1)$ como $l_{P}=\sqrt{\hbar}$.

Este resultado é compatível com a proposta de quantização da área e a relação termodinâmica, $S=A / 4 \hbar$, o que pode ser demonstrado como se segue. Temos que o número de estados $g(n)$ acessíveis a uma dada geometria, é inteiro e se relaciona com a entropia como $S=\ln g(n)$, de maneira que $g=e^{A / 4 \hbar}[39]$. Assim, necessariamente a quantização da área precisa ter o formato $A=4 n \hbar \ln C$, com $C$ constante, de maneira que $g$ permaneça inteiro.

Em sua conjectura, Hod propõe que esta constante seja $C=3$, tendo uma interpretação estatística (dada no último parágrafo) e que se compatibiliza com o princípio da correspondência de Bohr, de que frequências de transições em números quânticos grandes devem ser compatíveis com frequências de oscilação clássicas (o que pode ser observado através da comparação do espectro quasi-normal assintótico com a proposta do quantum de área em (3.3)).

A conjectura contudo apresenta algumas dificuldades. Primeiro, o espectro assintótico dos modos quasi-normais é naturalmente diferente, para diferentes buracos negros. Entretanto para que esta continue válida esperamos que no limite em que estes buracos negros se aproximem da solução 
de Schwarzschild, também recuperemos o espectro assintótico deste buraco negro. Para o buraco negro de Kerr, no entanto, temos que $\omega_{R}$ tende a zero conforme a rotação também se aproxima de zero com a proporção $\omega_{R} \propto a^{3}$ [40]. Desta maneira se a um buraco negro de Schwarzschild acrescentamos uma quantidade infinitesimal de rotação, o espectro assintótico é diferente, invalidando a conjectura: o quantum de área pode se tornar arbitrariamente pequeno nestas condições. Uma situação similar se aplica ao buraco negro de Reissner-Nordström.

Um outro aspecto problemático da conjectura é a relação de Motl-Neitzke para os modos assintóticos do buraco negro com carga [41],

$$
e^{8 \pi M \omega}=-1-2 \cos \pi j
$$

com $j$ o spin da perturbação. Neste caso, para as perturbações gravitacional e escalar, temos $\omega_{R}=T_{H}^{-1}$, o que não é verdade pra perturbações vetoriais e de spin semi-inteiro (para as quais $\omega_{R}=0$ ). Desta maneira, o valor assintótico de $\omega_{R}$ depende do tipo de perturbação analisada (ou do campo que se propaga), e não das propriedades intrínsicas do buraco negro, o que dificulta a interpretacão de $\omega_{R}$ como quantum de área.

Tais problemas bem como outros relacionados com a interpretação do spectro quasi-normal foram conceitualmente resolvidos em um trabalho publicado há dois anos, que será descrito na próxima seção.

\subsection{A Conjectura Hod Modificada}

Como citado na seção anterior, a conjectura de Hod possui alguns problemas conceituais. Um outro problema fundamental relacionado a ela, é o fato de a conjectura ser baseada, de um ponto de vista conceitual, em uma transição do buraco negro de um estado com valor alto de $n$ para o estado fundamental apenas (isto pelo ponto de vista de que um quantum de área corresponde à emissão de um modo em altas energias, com energia $\omega_{R}$ ). Uma transição de um estado assintótico $n$ para um também estado assintótico $n^{\prime}$ não é contemplado pela conjectura. Para uma transição $n \rightarrow n^{\prime}, \omega_{R}^{(n)-\left(n^{\prime}\right)} \propto O\left(1 / n^{1 / 2}\right)$, de 
Figura 3.1: Parte real dos modos quasi-normais de Schwarzschild para $l=2$ e $l=3$.

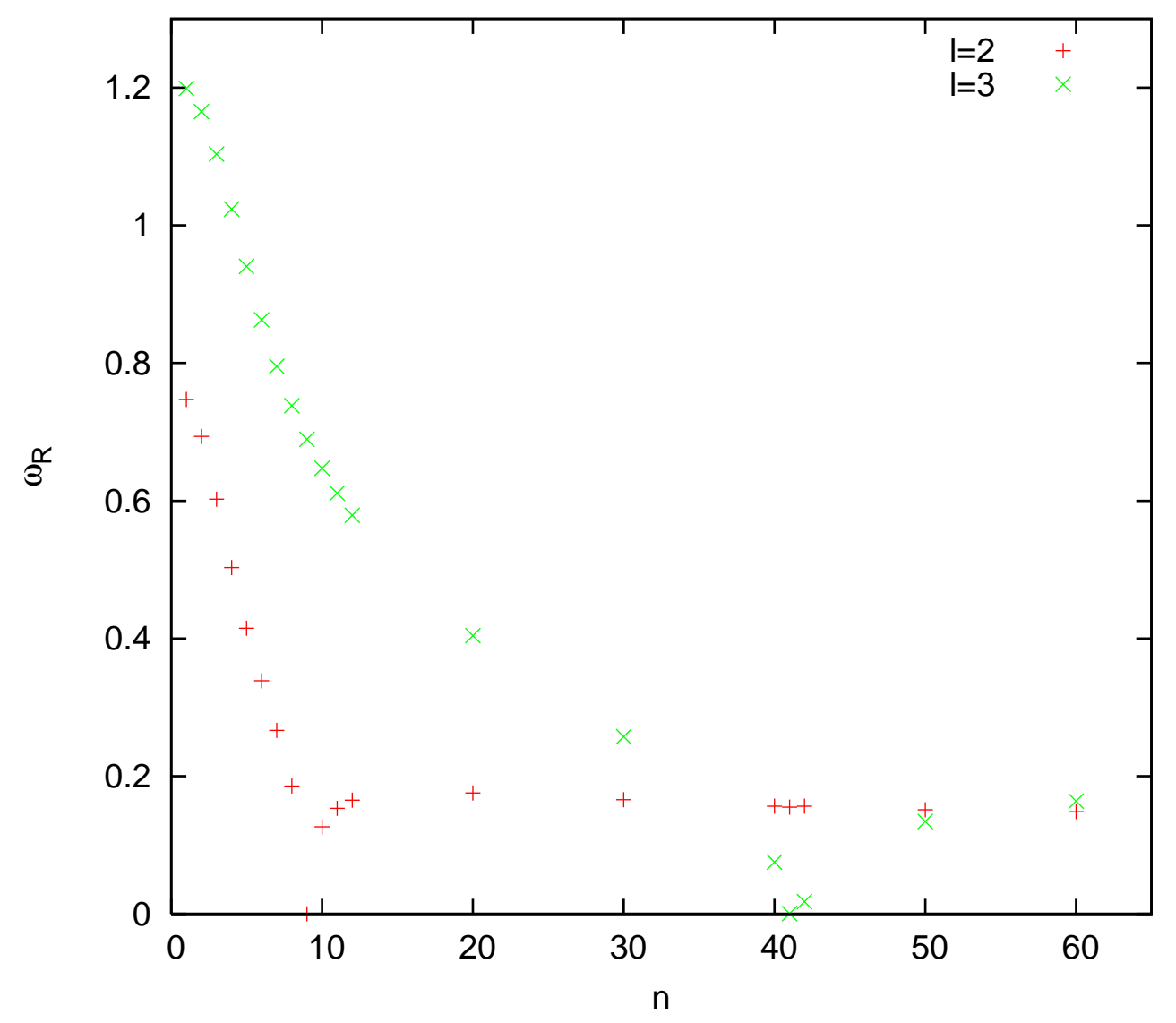

maneira que o quantum de área pode se tornar arbitrariamente pequeno.

Tal questão foi solucionada com a proposta de Maggiore de uma modificação na interpretação das grandezas físicas no espectro quasi-normal do buraco negro de Schwarzschild. Antes de falarmos propriamente da solução proposta por Maggiore [40], falaremos da física envolvendo os modos de perturbação do buraco negro.

O espectro da perturbação gravitacional da geometria de Schwarzschild [42] segue na figura 3.2.

O modo dominante no processo de emissão do buraco negro é o primeiro, pois é aquele com menor parte imaginária. Todos os demais modos tem parte imaginária crescente (com taxa de crescimento constante) de acordo 
com a progressão do número de overtone. Do ponto de vista astrofísico e observacional, os primeiros overtones são os mais importantes por terem menor fator de atenuação $\omega_{I}$, e neste sentido, terem maior possibilidade de serem observados em detectores e antenas gravitacionais [43, 44]. Os modos assintóticos por sua vez, (alto valor de $n$ ), respondem por uma possível conexão entre a oscilação da geometria e a termodinâmica do buraco negro, e por isso o interesse em calculá-los.

No gráfico 3.2, podemos observar o comportamento dos modos quasinormais para dois valores diferentes de momento angular, $l=2$ e $l=3$. Em ambos os casos, e também para outros valores de $l$ [34], a parte real inicialmente diminui até um dado modo $n_{c}$ e aumenta novamente a partir de $n_{c}$ aproximando-se do valor assintótico $\omega_{\infty}=T_{H} \ln 3$.

Comparado com sistemas clássicos tais como uma vareta vibrante tal tipo de resposta do buraco negro a perturbações em sua geometria é bastante peculiar. Isto porque em sistemas clássicos, o modo com menor amortecimento é em geral o modo com o menor valor de $\omega_{R}$, e tipicamente $\omega$ aumenta com o aumento de $n$ tanto em sua parte imaginária quanto real. De maneira diversa, na figura, até $n=n_{c}$, o valor de $\omega_{R}$ diminui, e ainda, a partir de $n_{c}$ este valor aumenta até atingir um ponto de saturação em $T_{H} \ln 3$. Em um sistema macroscópico normal, $\omega_{R}$ e $\omega_{I}$ aumentam indiscriminadamente com o aumento de $n$. Entretanto quando $\omega_{R}$ torna-se expressivamente grande, o comprimento de onda da oscilação associado, $\lambda=2 \pi \omega_{R}^{-1}$ torna-se expressivamente pequeno, e quando alcança o valor de distâncias de estruturas atômicas, a perturbação se dissipa como efeito de agitação termal da rede [40].

Em uma tentativa de explicação semi-clássica, a figura 3.2 deixa ainda questões sem esclarecimento. Em sistemas quânticos (em geral), os modos com energia maior $\left(\hbar \omega_{R}^{n \rightarrow \text { grande }}\right)$ decaem mais rapidamente, pois a largura de decaimento em uma expansão em multi-polos é proporcional a $\omega_{R}$, comportamento contrário ao da figura 3.2, para qual (em uma interpretação semiclássica) a probabilidade de decaimento de um modo diminui com o aumento de $n$ até o valor de $n_{c}$.

A tentativa de explicação sugerida em [40] é de comparar o buraco negro 
com um oscilador harmônico amortecido, sujeito a uma força 'tipo delta de Dirac',

$$
\ddot{X}+\gamma_{0} \dot{X}+\omega_{0}^{2} X=f(t)=\delta(t)
$$

Para tal sistema, as soluções são do tipo $e^{\omega_{ \pm} t}$, com $\omega_{ \pm}= \pm \sqrt{\omega_{0}^{2}-\gamma_{0}^{4} / 4}+$ $i \gamma_{0} / 2$. Desta maneira com as identificações $\omega_{I}=\gamma_{0} / 2$ e $\omega_{R}=\sqrt{\omega_{0}^{2}-\gamma_{0}^{2} / 4}$, conseguimos o comportamento de oscilação do tipo $e^{-\omega_{I} t}\left[\operatorname{asen}\left(\omega_{R} t\right)+b \cos \left(\omega_{R} t\right)\right]$. Neste caso, a frequência de oscilação dada por $\omega_{0}$ pode ser aproximada por $\omega_{R}$ para $n$ pequeno, uma vez que $\omega_{R} \gg \omega_{I}$. Contudo, distintamente, $\omega_{0} \sim \omega_{I}$ para $n$ grande, uma vez que

$$
\omega_{0}^{2}=\omega_{R}^{2}+\omega_{I}^{2}
$$

e $\omega_{I} \gg \omega_{R}$ para $n$ grande. Com a reinterpretação do espectro tendo $\omega_{0}$ como frequência de oscilação do buraco negro, os problemas descritos acima tanto em nível clássico como semi-clássico são resolvidos. Os espectros para $l=2$ e $l=3$ seguem na figura 3.2.

De acordo com esta figura, o modo com o menor valor de $n$ é também o com a menor frequência $\omega_{0}$. Também, a frequência aumenta monotonicamente com o aumento de $n$, como esperado. Ainda, uma atribuição sugestiva pode ser feita, em comparação com a relatividade restrira, se nomearmos $\hbar \omega_{0}^{\{n\}}=\sqrt{m_{0}^{2}+p_{n}^{2}}$, do que $m_{0}=T_{H} \ln 3$ e $p_{n}=2 \pi T_{H}(n+1 / 2)$. No caso, a expressão para $p_{n}$ é grandemente intrigante, uma vez que representa a mesma expressão para a quantização de uma partícula e um círculo de comprimento $L=\hbar T_{H}^{-1}[40,45]$. O espaçamento equidistante entre os diferentes níveis de $p_{n}$ é o exato esperado da descrição de um horizonte como uma membrana $[45]$.

Finalmente, a quantização da área pode ser facilmente obtida, considerando que a energia envolvida advem de uma transição de fase em altas energias, do gênero $n \rightarrow n-1$. Neste caso temos que $\omega_{0} \sim \omega_{I}$, do que

$$
\delta M=\hbar\left[\omega_{0}^{\{n\}}-\omega_{0}^{\{n-1\}}\right]=\hbar(4 M)^{-1} .
$$


Figura 3.2: Modos quasi-normais de Schwarzschild para $l=2,3$ em termos de $\omega_{0}$.

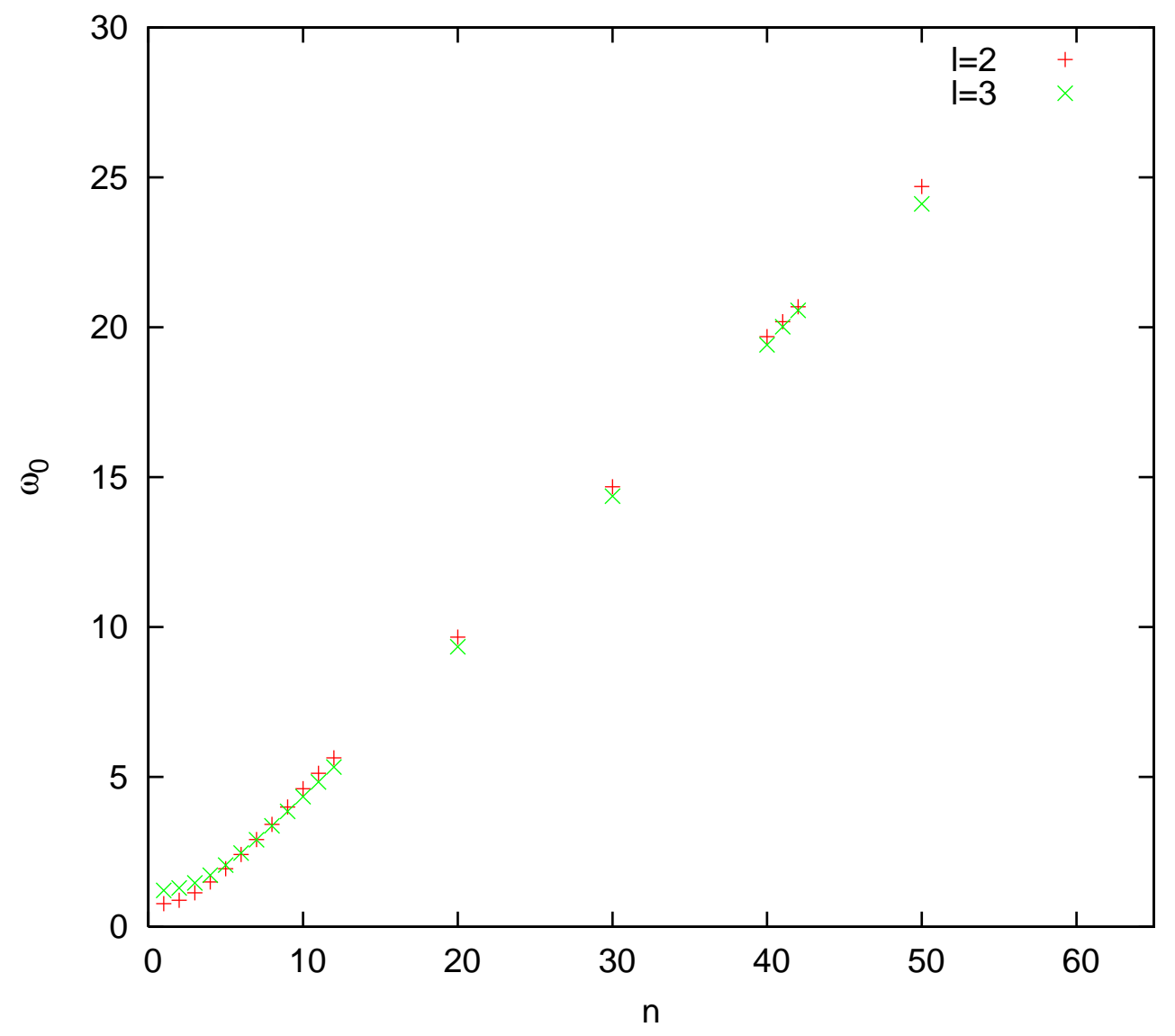


Neste caso, a quantização da área fica

$$
\delta A=32 \pi M \delta M=8 \pi \hbar=8 \pi l_{P}^{2}
$$

que é o resultado original proposto por Bekenstein na década de 70! Mesmo para transições a baixos valores de $n$, por exemplo de $n=2$ até $n=1$, temos um quantum de área sempre da ordem de $\delta A=C 8 \pi l_{P}^{2}$, com $C$ uma constante variando de 0 a 1 , que em tal caso vale 0.2 . O resultado de (3.8), não depende do spin do campo propagado na geometria como no caso da conjectura de Hod, de acordo com a relação de Motl-Neitzke, e os limites $n \rightarrow \infty$ e $Q, a \rightarrow 0$, evitando o problema também acima citado de invalidar a conjectura pela adição de pequenas quantidades de carga ou momento angular ao buraco negro.

A última questão 'proposta' na conjectura de Hod e que não pode ser equalizada pela modificação posterior, é a de que a quantização deve ter um formato $\delta A / \hbar=4 \ln k$, para que a contagem de microestados dada por $g(N)=e^{N \delta A}$ seja um número inteiro ${ }^{1}$. Entretanto, na aproximação semiclássica $N$ é um número bastante grande, o que faz com que $g$ seja ainda maior, invalidando portanto a possibilidade de se conseguir uma precisão na última casa de medida, para $g$, de uma unidade. Além disto, o quantum de área $\delta A$ é o mesmo independendo do valor de $n$, o que é conceitualmente injustificado, uma vez que a aproximação tomada, é válida apenas para altos valores de $n$ e portanto de $N$.

A fórmula da entropia com a constante que Bekenstein obteve para o quantum de área fica

$$
S=2 \pi N
$$

que é compatível com [46]. Do mesmo modo, em [41], tal propriedade é usada, para o cálculo dos modos quasi-normais, de maneira que tal propriedade (peridiocidade do tempo euclideano) parece estar relacionada não apenas à derivação da temperatura do buraco negro, bem como à quantização da área

\footnotetext{
${ }^{1}$ A grandeza $N$ aqui, não deve ser confundida $\operatorname{com} n$, sendo o fator de proporção entre a área de um buraco negro, e o quantum de área, $N=A / \delta A$.
} 
deste.

\subsection{Testando a conjectura com Buracos Ne- gros de Reissner-Nordström}

Ante a modificação proposta por Maggiore da conjectura de Hod, que motiva uma interpretação física mais coerente dos modos quasi-normais enquanto comportamento oscilatório, uma pergunta que pode ser colocada é se esta conjectura se mantém, para buracos negros de Reissner-Nordström [48].

Para o cálculo destas oscilações (perturbação gravitacional em ReissnerNordström), usamos o ferramental desenvolvido na próxima seção (nos ateremos aqui, a discutir os resultados).

Temos que a área do horizonte de eventos do buraco negro é dada por $A=4 \pi r_{+}^{2}$ e sendo o raio do horizonte $r_{+}=M\left[1+\sqrt{1-Q^{2} / M^{2}}\right]$, teremos que o quantum de área para este buraco negro será dado por

$$
\delta A=\frac{8 \pi r_{+}}{\sqrt{M^{2}-Q^{2}}}\left[\left(2 M-\frac{Q^{2}}{r_{+}}\right) \delta M-Q \delta Q\right]
$$

que, em termos de uma função $z$ definida como $z=Q / M$ fica

$\delta A=8 \pi M \frac{1+\sqrt{1-z^{2}}}{\sqrt{1-z^{2}}}\left[\left(2-\frac{z^{2}}{1+\sqrt{1-z^{2}}}\right) \delta M-z \delta Q\right] \equiv M[f(z) \delta M+g(z) \delta Q]$

Aqui estamos considerando a variação na área do buraco negro, através de uma emissão gravitacional que carregue para o infinito apenas energia. Portanto é razoável supor que, $\delta Q=0$. Neste caso, a expressão mandatória no processo de quantização é $M f(z) \delta M$. Contudo, para buracos negros cuja carga não se aproxime do valor extremo $(z=1), f(z) \sim 32 \pi$ pode ser tido como uma boa aproximação. Por exexemplo, se $Q$ tomar $60 \%$ do valor máximo, ou $z=0.6$, o erro na expressão acima é da ordem de $1 \%$. Para $Q$ próximo a $80 \%$ do valor máximo, o erro é de $6,5 \%$, o que torna viável esta aproximação, contanto que não nos aproximemos de $Q=Q_{\max }=M$. Neste 


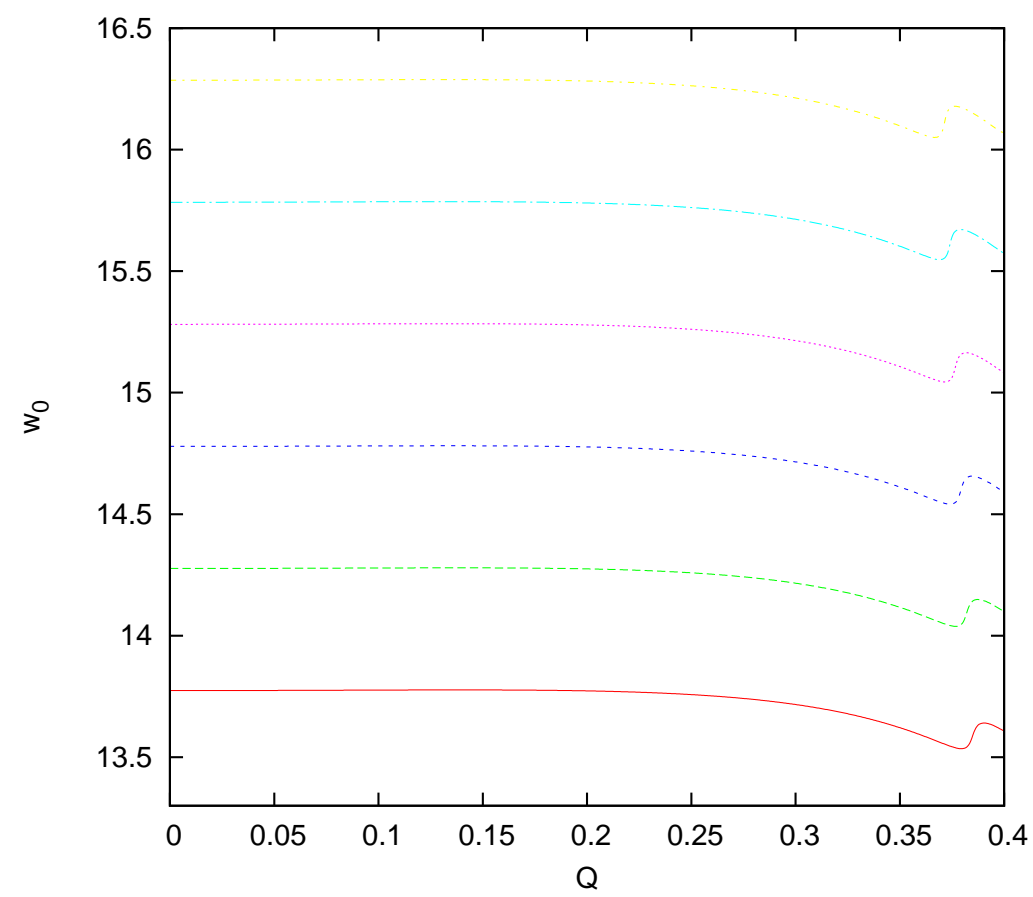

Figura 3.3: $\omega_{0}$ como função de $Q$, para a série $l=4$ e $n=28$ até $n=33$.

caso, o quantum de área é o mesmo que pra Schwarzschild (3.8), dado por

$$
\delta A_{R N} \sim 32 \pi M \delta M
$$

Sendo $\delta M$ a quantidade de energia ejetada no processo, $\delta M=\hbar \delta \omega_{0}$, e portanto, se $\delta \omega_{0}=\frac{1}{4 M}$, teremos o mesmo quantum de área que no caso de Schwarzschild, $\delta A=8 \pi l_{P}^{2}$. O trabalho portanto é o de verificar se $\delta \omega_{0}=$ $\frac{1}{2}$ (em todos os cálculos do resto deste capítulo, a menos que $M$ apareça explicitamente, adotamos $M=1 / 2$ ).

Para o largo espectro quasi-normal calculado, desde $l=2$ até $l=6$, tal proposição foi confirmada exceto por pequenas oscilações em determinadas regiões dos gráficos de $\omega_{0} \times Q$.

Um gráfico típico de $\omega_{0}$, para diferentes valores de $n$, e $l=4$ pode ser visto na figura (3.3). Embora não seja perceptível pela figura, há uma pequena 'diferença de fase', entre os gráficos de $\omega_{0}$ para diferentes valores de $n$. O 


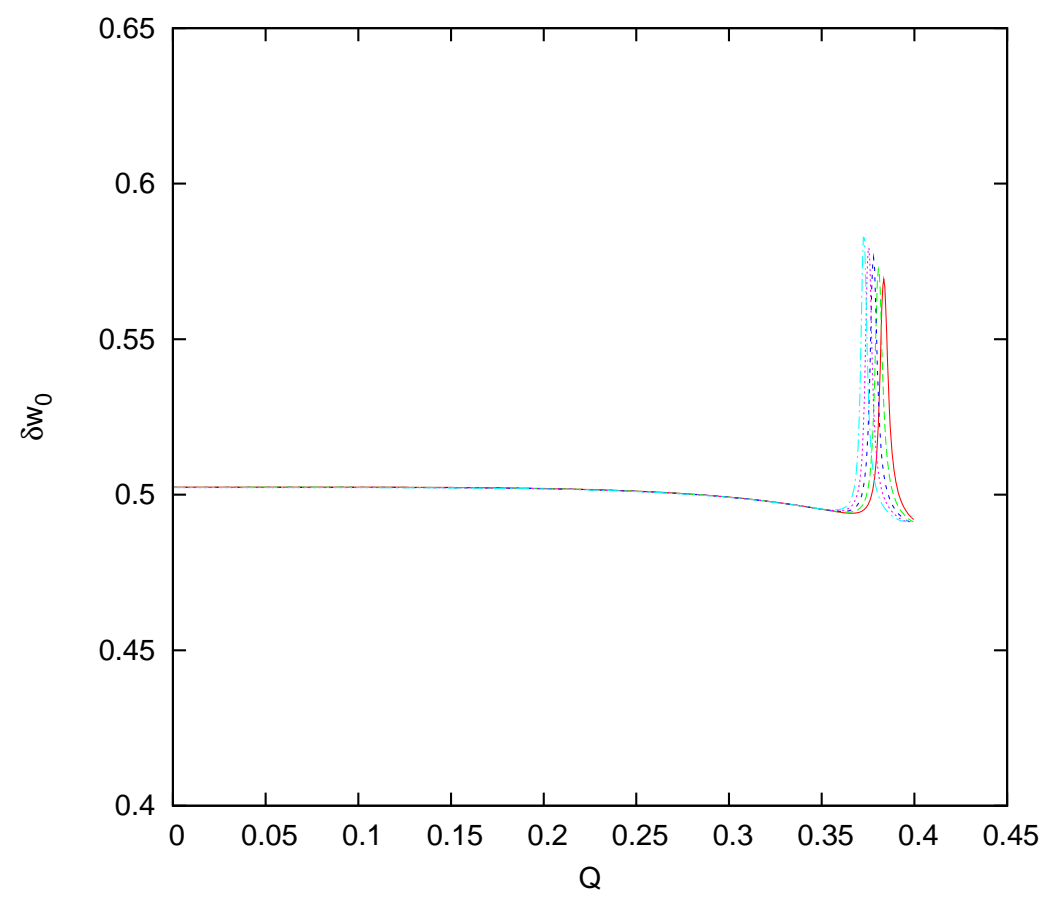

Figura 3.4: $\delta \omega_{0}$ como função de $Q$, para a série $l=4$ de $n=28$ até $n=33$.

correspondente gráfico de $\delta \omega_{0}$ x $Q$ é dado em (3.4).

Outra comparação também é feita no caso de $l=3$ nos gráficos $(3.5,3.6)$.

Em ambos os casos (e também para os demais valores de $l$ ), há uma diferença de fase entre o gráfico de $\omega_{0}$ para um dado $n$ e seu consecutivo $n-1$. A pergunta fundamental é se este comportamento é robusto, ou advem do fato de que $n$ ainda não é suficientemente grande para que possa expressar o comportamento assintótico adequado ${ }^{2}$.

Entretanto, se dermos um zoom na figura (3.4), é possível perceber que a largura desta diferença de fase diminui, à medida em que avançamos no valor de $n$ (figura 3.9): para $n=28,29$ (primeiro pico, da direita para a esquerda) o pico é mais largo do que para $n=32,33$ (último pico).

A questão remanescente é se os picos de diferença de fase diminuem, quando vamos a um $n$ suficientemente alto, ou continuam a crescer. Tal

\footnotetext{
${ }^{2}$ Pela limitação do método em si, $n$ 's grandes, muito maiores do que 100, em geral são possíveis apenas de se calcular até $z \sim 0.2$
} 

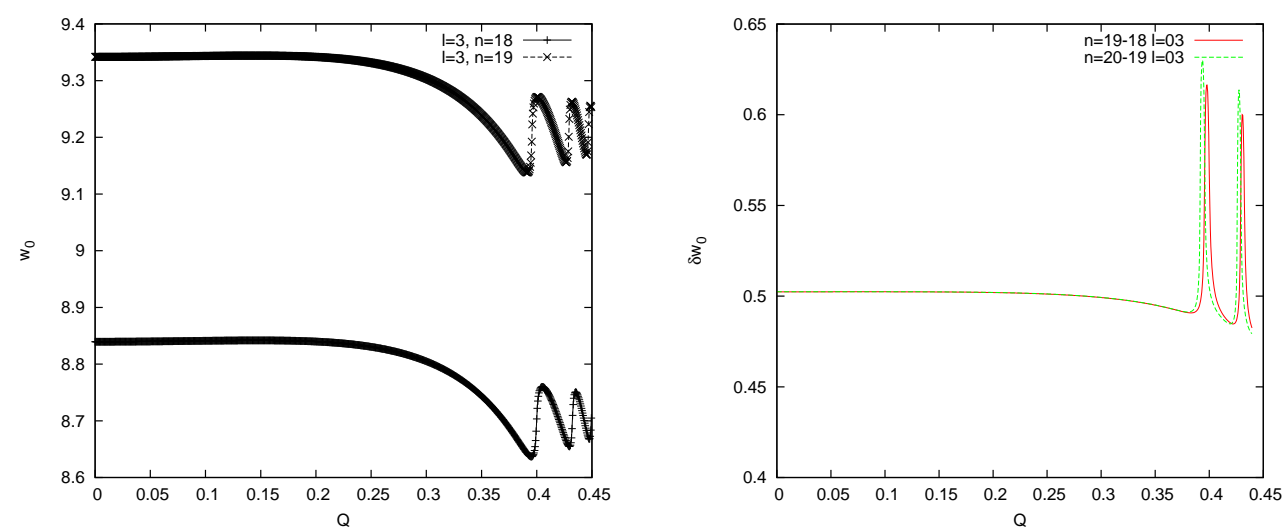

Figura 3.5: $\omega_{0}$ para $l=3$ e $n=$ Figura 3.6: $\delta \omega_{0}$ para $l=3$ e $n=$ 18, 19 na perturbação gravitacional. 18,19 na perturbação gravitacional.
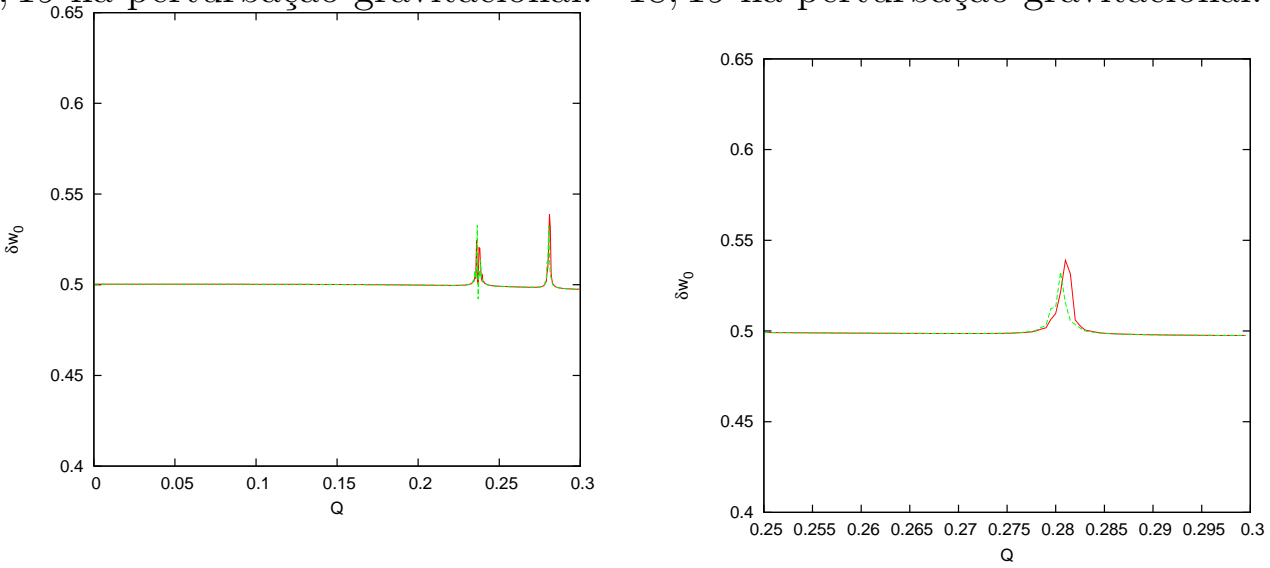

Figura 3.7: $\delta \omega_{0}$ para $l=3$ e $n=$ 200 - 202 na perturbação gravitaci- Figura 3.8: Zoom do gráfico ao lado. onal.

questão pode ser resolvida, observando-se os gráficos com $n=200$ e $l=3$ das figuras (3.7) e (3.8). Não apenas os picos diminuem em largura com o aumento de $n$, como também, diminuem em altura, para um $n$ grande.

Desta maneira, os resultados calculados sustentam a conjectura de Hod como modificada por Maggiore, para os buracos negros de Reissner-Nordström com cargas não próximas do valor extremo, $Q \sim M$.

O teste para tais valores de $Q$, contudo não pode ser feito, uma vez que o método de Leaver (descrito nas próximas seções), perde precisão em tal regime, uma vez que a expansão para o cálculo é feito através da variável 


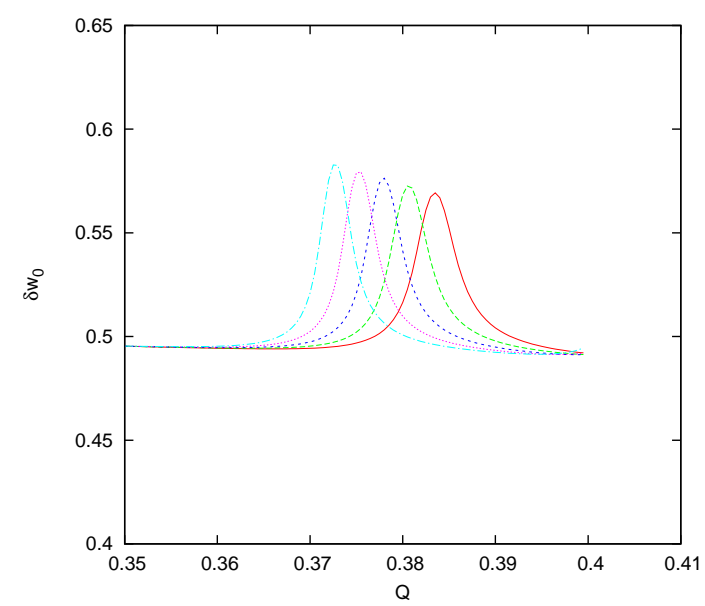

Figura 3.9: Zoom em $\delta \omega_{0}$ para $l=4$.

$u=\frac{r-r_{+}}{r-r_{-}}$, que tende a 1 , quando $Q \sim M$, pois neste caso $r_{-} \rightarrow r_{+} \cdot$

\subsection{Perturbações em Reissner-Nordström}

Outro aspecto da relação entre buracos negros e termodinâmica de buracos negros é uma proposta recente que associa uma mudança de fase de segunda ordem no buraco negro com carga e a termodinâmica [49, 47]. Nesta seção focaremos na perturbação gravitacional e na propagação de um campo de Dirac na geometria de Reissner-Nordström .

Tal geometria representa um buraco negro (ou a parte exterior a uma estrela) com massa e carga (portanto $T_{\mu \nu} \neq 0$ ), esfericamente simétrico em 4 dimensões, com elemento de linha dado por

$d s^{2}=-\left[1-\frac{2 M}{r}+\frac{Q^{2}}{r^{2}}\right]^{-1} d t^{2}+\left[1-\frac{2 M}{r}+\frac{Q^{2}}{r^{2}}\right] d r^{2}+r^{2} d \theta^{2}+r^{2} \operatorname{sen}^{2} \theta d \phi^{2}$

em que $Q$ representa a carga e $M$ a massa do buraco negro, como percebidas por um observador externo. Uma dedução razoável de tal elemento de linha pode ser encontrada em [50] (ou [51]), e não a daremos neste traba- 
lho. Também a estrutura conforma do espaço-tempo, bem como o estudo de geodésicas pode ser encontrado em [51].

\subsubsection{Perturbação Gravitacional}

Estamos interessados aqui inicialmente no cálculo de uma perturbação gravitacional $h$ na geometria $g$, escrito como

$$
g_{\mu \nu}^{p e r t}=g_{\mu \nu}+h_{\mu \nu},
$$

em que $g^{\text {pert }}$ representa a métrica original. Os trabalhos originais a respeito de perturbação gravitacional remetem a Regge e Wheeler na década de 50 [4], em um artigo no qual se calcula a perturbação da geometria de Schwarzschild, demonstrando que o horizonte é estável a perturbações em primeira ordem (i. e., tais perturbações permanecem pequenas com a evolução temporal).

Em geral o tensor $h$ é escrito levando-se em consideração as simetrias da métrica em questão. Por exemplo, para a geometria de Schwarzschild é possível em um gauge específico [4] termos apenas dois termos não nulos (e seus correspondetens simétricos), que dependem essencialmente de $(r, t)$, tornando as equações mais simples de serem integradas ${ }^{3}$.

Na geometria com massa e carga (Reissner-Nordström), as perturbações gravitacional e eletromagnética se acoplam, visto que em ordem zero na equação de Einstein, $T_{\text {materia }}$ é nulo, e apenas temos $T_{\text {eletromagnetico }} \neq 0$. Além das equações de Einstein, temos as equações de Mawell, formando um total de 14 equações $[52,53]$,

$$
\begin{array}{r}
G_{\mu \nu}=8 \pi\left(T_{\mu \nu}+E_{\mu \nu}\right), \\
\nabla_{\mu}\left[\sqrt{-g} F^{\mu \nu}\right]-4 \pi \sqrt{-g} J^{\mu}=0 .
\end{array}
$$

$E$ representando o tensor energia-momento eletromagnético e $T$ este tensor para a matéria (que em ordem zero é nulo). A perturbação introduzida via $h$ como em (3.14), afeta também o tensor de Maxwell, de maneira que as

\footnotetext{
${ }^{3}$ Embora, não sem uma perda intuitiva da interpretação do sistema de coordenadas $[15]$.
} 
equações de perturbação são escritas como

$$
\begin{array}{r}
\delta G_{\mu \nu}=8 \pi\left(T_{\mu \nu}+\delta E_{\mu \nu}\right) \\
\delta\left[\nabla_{\nu}\left(\sqrt{-g} F^{\mu \nu}\right)\right]-4 \pi \sqrt{-g} J^{\mu}=0
\end{array}
$$

uma vez que $J$ também é nulo em ordem zero (ausência de correntes na solução estática).

Há dois tipos básicos de perturbação, como na métrica de Schwarzschild: com paridade par (polar) ou ímpar (axial), quanto à transformação de $h$ com relação a sua decomposição em harmônicos esféricos tensoriais. Tanto a perturbação em $g$ quanto em $F$ se dividem em duas partes nomeadas de multipolos magnéticos e elétricos, que associamos conceitualmente aos dois graus de liberdade do gráviton em 4 dimensões [54]. Ainda cada uma destas perturbações é composta de dois graus de liberdades diferentes expressos em seu potencial, responsáveis pelos dois tipos físicos de perturbação introduzidos no cálculo: eletromagnético e gravitacional. No buraco negro com carga, estas duas perturbações estão acopladas, o que não ocorre em Schwarzschild.

Uma derivação passo a passo das perturbações gravitacional e eletromagnética, tanto com paridade polar quanto axial pode ser encontrada em [51] (ou em $[52,53]$ ). Dada a extensão de tais cálculos já bem conhecidos na literatura, nos limitaremos aqui a tomar a equação (axial) em sua forma final,

$$
\left[\frac{d^{2}}{d r_{*}^{2}}+\omega^{2}-V(r)\right] \Psi(r)=0
$$

em que $r_{*}$ representa a coordenada tartaruga, calculada como

$$
d r_{*}=g_{11} d r \rightarrow \quad r_{*}=r+\frac{r_{+}^{2} \ln \left(r-r_{+}\right)-r_{-}^{2} \ln \left(r-r_{-}\right)}{r_{+}-r_{-}}
$$

com $r_{ \pm}=\left[1 \pm \sqrt{1-4 Q^{2}}\right] / 2$ representanto os horizontes de eventos (externo) e de Cauchy (interno). Ainda, $\omega$ é a frequência advinda de uma dependência 
temporal do tipo $\psi=e^{-i \omega t}$ e $V(r)$ o potencial axial escrito como

$$
V(r)=\frac{g^{11}}{r^{4}}\left[l(l+1) r^{2}-\Gamma r+4 Q^{2}\right]
$$

$\operatorname{com} 2 \Gamma=3 \pm \sqrt{9+16 Q^{2}(l-1)(l+2)}$. Os sinais $+\mathrm{e}-$ acontecem por serem duas as perturbações tratadas, gravitacional e eletromagnética. No limite em que $Q$ é nulo, e recuperamos a geometria de Schwarzschild, a equação (3.18) é a perturbação axial gravitacional se tomamos o sinal positivo em $\Gamma$, e a equação de perturbação eletromagnética axial, ao tomarmos o sinal negativo.

A paridade polar de perturbação é descrita por uma equação cuja função do campo, $\Phi(r)$ acopla com a função de paridade axial,

$$
\begin{array}{r}
{[(l-1) l(l+1)(l+2)+2 i \omega \Gamma] \Phi(r)-2 \Gamma \frac{d \Psi(r)}{d r_{*}}} \\
-\left[(l-1) l(l+1)(l+2)+\frac{2 \Gamma^{2} g^{11}}{(l-1)(l+2) r^{2}+\Gamma r}\right] \Psi(r)=0
\end{array}
$$

Estamos interessados aqui no espectro quasi-normal da equação, o que representa resolver (3.18) com condições de contorno específicas de ondas planas nos infinitos espaciais,

$$
\lim _{r_{*} \rightarrow \pm \infty} \Psi(r) \propto e^{\mp i \omega r_{*}}
$$

Há diversas maneiras de se fazer a integração numérica deste problema, utilizando-se de diferentes métodos disponíveis na literatura. Um método bastante utilizado pra tal efeito é o método WKB [55], similar ao utilizado em mecânica quântica básica. O problema da aproximação WKB é o fato de esta perder precisão para altos valores do número de overtone $n$, ainda que a tomemos até sexta ordem ${ }^{4}[56]$. Uma correção para tal problema pode se encontrada em [58].

Outro possibilidade para o cálculo é a utilização do método de Leaver

\footnotetext{
${ }^{4}$ De fato, o método WKB mantem-se uma boa aproximação para os modos quasinormais até $n \sim l$ [57]. Quando $n \geq l$, devemos empregar o método da integral no espaço de fases [58].
} 
(Frobenius), publicado originalmente na década de 80 [59, 60]. Faremos uso aqui deste método, pela relativa facilidade em se calcular modos com altos valores de $n$.

\subsubsection{O método de Leaver (Frobenius) - frações parci- ais}

Inicialmente, vamos expandir a função $\Psi$ em termos de um somatório de uma variável convenientemente escolhida [60], $u=\frac{r-r_{+}}{r-r_{-}}$,

$$
\Psi(r)=\frac{r_{+}\left(r_{+}-r_{-}\right)^{i \omega+1} u^{b}}{r\left(r_{+}-r_{-}\right)^{2 i \omega+1} e^{i \omega\left(r+2 r_{+}\right)}} \sum_{n=0}^{\infty} a_{n} u^{n}
$$

em que $b\left(r_{+}-r_{-}\right)=-i \omega r_{+}^{2}$. Os limites da equação (3.22) quando usados no Ansatz acima resultam em

$$
\begin{aligned}
\lim _{r_{*} \rightarrow-\infty} \Psi(r) & =\left[e^{-i \omega r_{+}}\left(r_{+}-r_{-}\right)^{\frac{i \omega r_{-}^{2}}{r_{+}-r_{-}}}\right]\left(r-r_{+}\right)^{b}, \\
\lim _{r_{*} \rightarrow+\infty} \Psi(r) & =\left[r_{+} e^{-2 i \omega r_{+}}\left(r_{+}-r_{-}\right)^{-2 i \omega-1} \sum_{n=0}^{\infty} a_{n}\right] e^{i \omega r_{*}}
\end{aligned}
$$

do que devemos ter que $\sum a_{n}$ converge (para que possamos usar este ansatz). E com tal expansão para $\Psi$, substituída na equação de onda, obtemos uma relação de recorrência com 3 equações definidas a seguir,

$$
\begin{aligned}
& 0=\alpha_{0} a_{1}+\beta_{0} a_{0} \\
& 0=\alpha_{1} a_{2}+\beta_{1} a_{1}+\gamma_{1} a_{0} \\
& 0=\alpha_{n} a_{n+1}+\beta_{n} a_{n}+\gamma_{n} a_{n-1}+\delta_{n} a_{n-2} .
\end{aligned}
$$


em que os coeficientes $\alpha, \beta, \gamma$ e $\delta$ são escritos em termos de $M, Q, \Gamma, n$ e $\omega$ como

$$
\begin{aligned}
\alpha_{n}= & {\left[n^{2}+2(b+1) n+2 b+1\right] r_{+} } \\
\beta_{n}= & \left(-2+r_{-}\right) n^{2}+\left[-2-2 b\left(2-r_{-}\right)+4 i \omega r_{+}^{2}+6 r_{-}\right] n+\left[\Gamma+2 \omega^{2} r_{+}^{3}\right. \\
& \left.+4 i \omega r_{+}^{2}(1+b)-2 b^{2}\left(2-r_{+}^{-1}\right)-r_{+} l(l+1)-(3-2 b) r_{-}\right] \\
\gamma_{n}= & \left(1+r_{-}\right) n^{2}+\left[-2 i \omega r_{+}\left(1+2 r_{-}\right)+2 b\left(1+r_{-}\right)-10 r_{-}\right] n+ \\
& -i \omega r_{+}\left[2-12 r_{-}+(-i \omega+2 b)\left(1+2 r_{-}\right)\right]-1-\Gamma-2 b\left(1+3 r_{-}\right) \\
& +b^{2}\left[16+8 r_{-}-\left(15-38 r_{-}+26 r_{-}^{2}\right) r_{+}^{-3}\right]+\left(l^{2}+l+13\right) r_{-} \\
\delta_{n}= & {\left[-n^{2}+2(3+i \omega-b) n-(9-4 i \omega b-6 b+6 i \omega)\right] r_{-} }
\end{aligned}
$$

em que escolheremos por conveniência $a_{0}=1$. Para resolver estas equações e encontrar os modos quasi-normais, faremos inicialmente uma digressão para o problema semelhante de Schwarzschild [59], ou seja, no limite em que $Q=0$. Em tal situação, $\delta_{n}=0$ e teremos uma relação de recorrência envolvendo apenas três coeficientes genéricos $\alpha, \beta$ e $\gamma$, além de apenas duas equações, a saber (3.27) e (3.29) uma vez que (3.27) é como (3.28) com $\delta=0$ e $n \rightarrow n-1$.

No caso de Schwarzschild, a equação de onda é escrita como

$$
r(r-2 M) \frac{\partial^{2} \psi}{\partial r^{2}}+\frac{\partial \psi}{\partial r}-\left[-\frac{\omega^{2} r^{3}}{r-2 M}+l(l+1)-\frac{2 M\left(1-s^{2}\right)}{r}\right] \psi=0
$$

As condições de contorno neste caso são expressas sob a forma

$$
\lim _{r \rightarrow 2 M} \rightarrow(r-2 M)^{-i \omega}, \quad \quad \lim _{r \rightarrow \infty} \rightarrow r^{i \omega} e^{i \omega r}
$$

e o Ansatz para a solução em $\psi$ é o mesmo tomado pra Reissner-Nordström, com $Q=0$, ou seja,

$$
\psi=(r-2 M)^{-i \omega} r^{2 i \omega} e^{i \omega(r-2 M)} \sum_{n=0}^{\infty} a_{n}\left[\frac{r-2 M}{r}\right]^{n} .
$$

Com estas colocações, o problema em Schwarzchild torna-se extremamente similar ao de Reissner-Nordström, exceto que temos uma equação de re- 
corrência a mais (bem como um termo extra) neste caso. A relação para o limite assintótico de $a_{n}$ é dada por [60]

$$
\lim _{n \rightarrow \infty} \frac{a_{n+1}}{a_{n}} \sim 1-\sqrt{\frac{-i \omega}{n}}+\frac{-2 i \omega-3 / 4}{n}+T\left(n^{-3 / 2}\right)
$$

e a série pode ser resolvida em termos de uma fração parcial, escrita como

$$
\frac{a_{n+1}}{a_{n}}=-\frac{\gamma_{n+1}}{\beta_{n+1}-\frac{\alpha_{n+1} \gamma_{n+2}}{\beta_{n+2}-\frac{\alpha_{n+2} \gamma_{n+3}}{\beta_{n+3}-\cdots}}}
$$

que usualmente é representada como

$$
\frac{a_{n+1}}{a_{n}}=-\frac{\gamma_{n+1}}{\beta_{n+1}-} \frac{\alpha_{n+1} \gamma_{n+2}}{\beta_{n+2}-} \frac{\alpha_{n+2} \gamma_{n+3}}{\beta_{n+3}-} \ldots
$$

Com algumas manipulações algébricas, obtemos a forma final que pode ser iterada indefinidamente, fazendo-se uso da expressão (3.37) para o final da série,

$$
\left[\beta_{n}-\frac{\alpha_{n-1} \gamma_{n}}{\beta_{n-1}-} \frac{\alpha_{n-2} \gamma_{n-1}}{\beta_{n-2}-} \ldots-\frac{\alpha_{0} \gamma_{1}}{\beta_{0}}\right]=\left[\frac{\alpha_{n} \gamma_{n+1}}{\beta_{n+1}-} \frac{\alpha_{n+1} \gamma_{n+2}}{\beta_{n+2}-} \frac{\alpha_{n+2} \gamma_{n+3}}{\beta_{n+3}-} \ldots\right]
$$

Para usar estes artifícios no caso de Reissner-Nordström, precisamos reduzir as relações de recorrência a duas equações, como no caso de Schwarzschild. Uma maneira simples de conseguir este efeito é redefinir os coeficientes $\alpha, \beta, \gamma$ e $\delta$ de maneira que

$$
\begin{aligned}
& 0=\alpha_{0}^{\prime} a_{1}+\beta_{0}^{\prime} a_{0} \\
& 0=\alpha_{n}^{\prime} a_{n+1}+\beta_{n}^{\prime} a_{n}+\gamma_{n}^{\prime} a_{n-1} .
\end{aligned}
$$


A redefinição proposta é dada por

$$
\begin{aligned}
\delta_{n}^{\prime} & =0 \\
\alpha_{n}^{\prime} & =\alpha_{n} \\
\beta_{n}^{\prime} & =\beta_{n}-\frac{\alpha_{n-1}^{\prime} \delta_{n}}{\gamma_{n-1}^{\prime}} \\
\gamma_{n}^{\prime} & =\gamma_{n}-\frac{\beta_{n-1}^{\prime} \delta_{n}}{\gamma_{n-1}^{\prime}} .
\end{aligned}
$$

para $n \geq 2$, e por

$$
\alpha_{n}^{\prime}=\alpha_{n}, \quad \beta_{n}^{\prime}=\beta_{n}, \quad \gamma_{n}^{\prime}=\gamma_{n}
$$

para $n=0,1$.

Temos agora todo o ferramental necessário para a obtenção dos modos quasi-normais gravitacionais de acordo com o método de Leaver. Nas próximas subseções analisaremos as perturbações de Dirac e escalar.

\subsubsection{Propagação dos Campos de Spin meio e Escalar na geometria de Reissner-Nordström}

A equação do campo de spin 1/2 tem um par de equações em termos do formalismo de Newman-Penrose escrito como [51]

$$
\begin{aligned}
& \sigma_{A B}^{i} \nabla_{i} P^{A}+i \mu Q^{C} \varepsilon_{C B}=0 \\
& \sigma_{A B}^{i} \nabla_{i} Q^{A}+i \mu P^{C} \varepsilon_{C B}=0,
\end{aligned}
$$

em que $\mu \sqrt{2}$ é a massa da partícula, $P, Q$ um par de espinores que representam a função de onda e $\sigma$ são as matrizes de Pauli dadas em termos da tétrada de Newman-Penrose,

$$
\sigma_{A B}^{i}=\frac{1}{\sqrt{2}}\left[\begin{array}{cc}
l^{i} & m^{i} \\
m^{i} & n^{i}
\end{array}\right] .
$$


Escrevendo em termos dos coeficientes de spin, e das componentes dos espinores, $\mathbf{P}=\left(F_{1}, F_{2}\right), \mathbf{Q}=\left(-G_{2}, G_{1}\right)$, obtemos o grupo de equações

$$
\begin{aligned}
(D+\varepsilon-\rho) F_{1}+\left(\delta^{*}+\pi-\alpha\right) F_{2} & =i \mu G_{1} \\
(\Delta+\mu-\gamma) F_{2}+(\delta+\beta-\tau) F_{1} & =i \mu G_{2} \\
\left(D+\varepsilon^{*}-\rho^{*}\right) G_{2}-\left(\delta+\pi^{*}-\alpha^{*}\right) G_{1} & =i \mu F_{2} \\
\left(\Delta+\mu^{*}-\gamma\right) G_{1}-\left(\delta^{*}+\beta^{*}-\tau^{*}\right) G_{2} & =i \mu F_{1} .
\end{aligned}
$$

As equações acima são bastante genéricas e valem para um grande número de diferentes espaços-tempo (por exemplo Kerr e Kerr-Newman). Vamos particularizar para o caso de Reissner-Nordström ao escrevermos os coeficientes de spin em termos do sistema de coordenadas esférico e da solução como escrita no começo da seção (3.13). Em tal caso, os coeficientes de spin usados nas equações de campo assumem as expressões [51]

$$
\begin{aligned}
& \kappa=\sigma=\lambda=\nu=\varepsilon=\pi=\tau=0, \\
& \rho=-\frac{1}{r}, \quad \beta=\alpha=\frac{\operatorname{cotg} \theta}{\sqrt{8} r}, \\
& \mu=-\frac{r^{2}-2 M r+Q^{2}}{2 r^{3}}, \quad \gamma=\frac{M r-Q^{2}}{2 r^{3}}
\end{aligned}
$$

Para as tétradas, quando projetadas no sistema de referências esférico temos que

$$
\begin{aligned}
D & =l=\mathbb{D}_{0}, \quad \Delta=n=-\frac{r^{2}-2 M r+Q^{2}}{2 r^{2}} \mathbb{D}_{0}^{\dagger} \\
\delta & =m=\frac{\mathbb{L}_{0}^{\dagger}}{r \sqrt{2}}, \quad \delta^{*}=\bar{m}=\frac{\mathbb{L}_{0}}{r \sqrt{2}}
\end{aligned}
$$

em que os operadores genéricos $\mathbb{D}, \mathbb{L}$ são escritos como

$$
\begin{aligned}
& \mathbb{D}_{n}=\partial_{r}+\frac{i \omega r^{2}}{r^{2}-2 M r+Q^{2}}, \quad \mathbb{D}_{n}^{\dagger}=\mathbb{D}_{n}^{*}, \\
& \mathbb{L}_{n}=\partial_{\theta}+n \operatorname{cotg} \theta+m \operatorname{cosec} \theta, \quad \mathbb{L}_{n}^{\dagger}=\partial_{\theta}+n \operatorname{cotg} \theta-m \operatorname{cosec} \theta
\end{aligned}
$$


Para obter uma equação de propagação do campo, finalmente, faremos duas mudanças de variáveis de maneira que estas 4 equações sejam separáveis e representem partículas de spin $1 / 2$ e $-1 / 2$. Notadamente, redefinimos as funções $F$ e $G$,

$$
\begin{aligned}
\left(F_{1}, F_{2}, G_{1}, G_{2}\right) & =\left(f_{1} / r, f_{2}, g_{1}, g_{2} / r\right) \\
\left(f_{1}, f_{2}, g_{1}, g_{2}\right) & =\left(R_{-} S_{-}, R_{+} S_{+}, R_{+} S_{-}, R_{-} S_{+}\right) .
\end{aligned}
$$

com o que as equações (3.51) ficam como

$$
\begin{aligned}
S_{-} \mathbb{D}_{0} R_{-}+\frac{1}{\sqrt{2}} R_{+} \mathbb{L}_{1 / 2} S_{+} & =0, \\
h S_{+} \mathbb{D}_{1 / 2}^{\dagger} R_{+}-\sqrt{2} R_{-} \mathbb{L}_{1 / 2}^{\dagger} S_{-} & =0, \\
S_{+} \mathbb{D}_{0} R_{-}-\frac{1}{\sqrt{2}} R_{+} \mathbb{L}_{1 / 2} S_{+} & =0, \\
h S_{-} \mathbb{D}_{1 / 2}^{\dagger} R_{+}+\sqrt{2} R_{-} \mathbb{L}_{1 / 2}^{\dagger} S_{+} & =0 .
\end{aligned}
$$

sendo $h=r^{2}-2 M r+Q^{2}$, e tendo tomado $\mu=0$ (ou seja, para partículas não massivas). As designações $+\mathrm{e}-$ nas funções de onda representam partículas de spin positivo e negativo no limite clássico, ou seja quando $g \rightarrow \eta_{\text {Minkowski }}$.

As equações acima podem ser separadas sem muito trabalho em parte angular, e parte radial,

$$
\begin{aligned}
0 & =\left(h \mathbb{D}_{1 / 2}^{\dagger} \mathbb{D}_{0}-\lambda^{2}\right) R_{-}, \\
0 & =\left(\mathbb{D}_{0} h \mathbb{D}_{1 / 2}^{\dagger}-\lambda^{2}\right) R_{+}, \\
0 & =\left(\mathbb{L}_{1 / 2}^{\dagger} \mathbb{L}_{1 / 2}+\lambda^{2}\right) S_{+}, \\
0 & =\left(\mathbb{L}_{1 / 2} \mathbb{L}_{1 / 2}^{\dagger}+\lambda^{2}\right) S_{-},
\end{aligned}
$$

e que feito o devido uso dos operadores $\mathbb{D}$ resulta, para a parte radial,

$$
\begin{gathered}
\left\{h \frac{\partial^{2}}{\partial r^{2}}+\frac{\dot{h}}{2} \frac{\partial}{\partial r}+\left[\omega^{2} \frac{r^{4}}{h}-i \omega\left(\frac{r^{2} \dot{h}}{2 h}+2 r\right)\right]-\lambda^{2}\right\} R_{-}=0,(3.59) \\
\left\{h \frac{\partial^{2}}{\partial r^{2}}+\frac{3 \dot{h}}{2} \frac{\partial}{\partial r}+\left[\omega^{2} \frac{r^{4}}{h}-i \omega\left(-\frac{r^{2} \dot{h}}{2 h}+2 r\right)\right]-\Lambda^{2}\right\} R_{+}=0,(3.60)
\end{gathered}
$$


$\operatorname{com} \Lambda^{2}=\lambda^{2}-1, \lambda^{2}=(j+1 / 2)(j+1 / 2)$ e ${ }^{5} j=l+1 / 2$. Estas equações quando integradas usando-se o método de Leaver - desenvolvido na última seção - nos dão, com uma precisão alta, os modos quasi-normais.

A propagação do campo escalar, segue a equação de Kelin-Gordon, que já especificamos no capítulo anterior ao trabalhar com a métrica de Ernst. A geometria é dada por $g_{\mu \nu}=\operatorname{diag}\left(-a, a^{-1}, r^{2}, r^{2} \operatorname{sen}^{2} \theta\right)$, com $a=h / r^{2}$, de maneira que o desenvolvimento de $\frac{1}{\sqrt{-g}} \partial_{\mu}\left[\sqrt{-g} g^{\mu \nu} \partial_{\nu} \Phi\right]=0$ leva a

$$
\left[h \frac{\partial^{2}}{\partial r^{2}}+\dot{h} \frac{\partial}{\partial r}+\omega^{2} \frac{r^{4}}{h}-l(l+1)\right] \Psi(r)=0 .
$$

Com efeito, uma análise das equações de propagação dos campos de diferentes spins, como apresentamos aqui, podem reduzida-las a uma única equação mestra, a saber

$$
\left[h \frac{\partial^{2}}{\partial r^{2}}+(s+1) \dot{h} \frac{\partial}{\partial r}+\omega^{2} \frac{r^{4}}{h}+i \omega s\left(r^{2} \frac{\dot{h}}{2 h}+4 r\right)-P^{2}\right] \Psi=0
$$

em que $s$ representa o spin do campo propagado e $P=L(L+1)-s(s+1) .{ }^{6}$ Desta maneira, o ansatz para resolver esta equação com o método de Leaver também é genérico e dado por

$$
\Psi=r\left(r-r_{+}\right)^{-s / 2-b}\left(r-r_{-}\right)^{-1-s / 2+2 i \omega+b_{-}} e^{i \omega\left(r-r_{-}\right)} \sum_{n=0}^{\infty} a_{n}\left[\frac{r-r_{+}}{r-r_{-}}\right]^{n}
$$

$\left(b_{-}=b r_{-}^{2} / r_{+}^{2}\right)$. As relações de recorrência ficam exatamente iguais as de (3.27-3.29), exceto pelo fato de que $\delta=0$. Os coeficientes usados para o cálculo da fração parcial e por conseguinte dos modos quasi-normais são

\footnotetext{
${ }^{5}$ Por uma questão de convenção usamos diferentes termos para a parte angular da equação de um campo de spin meio ou inteiro. Isto porque, para spin meio, o valor do momento angular pode variar de acordo com $j= \pm 1 / 2, \pm 3 / 2, \pm 5 / 2, \ldots$, e para spin zero, a variação é $l=0, \pm 1, \pm 2, \ldots$

${ }^{6}$ Respeitando a convenção adotada, $L=l$ para spin nulo, e $L=j$ para spin semi-inteiro.
} 
dados, em termos dos spins por

$$
\begin{aligned}
\alpha_{n} & =n^{2}+\left(C_{0}+1\right) n+C_{0}, \\
\beta_{n} & =-2 n^{2}+\left(C_{1}+2\right) n+C_{3}, \\
\gamma_{n} & =n^{2}+\left(C_{2}-3\right) n+C_{4}-C_{2},
\end{aligned}
$$

com as constantes $C^{\prime}$ 's determinadas por

$$
\begin{aligned}
& C_{0}=1-s-i \omega-i \omega K, \quad C_{1}=-4+2 i \omega(2+b)+2 i \omega K, \\
& C_{2}=s+3-3 i \omega-i \omega K, \quad C_{4}=s-1+2 i \omega(i \omega-s-3 / 2)-(2 \omega+i) \omega K, \\
& C_{3}=\omega^{2}\left(4+2 b-4 r_{-} r_{+}\right)-s-1+i \omega(2+b)-P+\omega K(2 \omega+i), \quad \\
& \left(K=\frac{r_{+}^{2}+r_{-}^{2}}{r_{+}-r_{-}}\right) .
\end{aligned}
$$

Com tais artifícios, já estaremos aptos a iterar a equação das frações parciais, que tem a mesma forma que (3.40), e obter os modos quasi-normais. $\mathrm{Na}$ próxima seção, motivaremos o cálculo do espectro quasi-normal para valores altos de $n$, através da proposição de uma relação entre termodinâmica e modos-quasi-normais.

\subsection{Mudança de Fase de Segunda Ordem em Buracos Negros e Modos Quasi-normais}

A mudança de fase de segunda ordem em buracos negros foi investigada primeiramente em [61], e está relacionada ao fato de que a capacidade térmica associada a buracos negros pode ser negativa, dependendo de seus parâmetros, ou positiva. Por exemplo, para Schwarzschild temos que $S=4 \pi M^{2}, T=$ $(8 \pi M)^{-1}$, levando a uma capacidade térmica negativa $C=T \frac{\partial S}{\partial T}=-8 M^{2}$, fato este bastante curioso que prenuncia uma termodinâmica não usual.

Em uma métrica bastante genérica, com constante cosmológica, carga, massa e momento angular, a entropia pode ser calculada como,

$$
S=\frac{1}{4} \int \sqrt{g_{\theta \theta} g_{\phi \phi}} d \theta d \phi=\pi \frac{r_{h}^{2}+a^{2}}{1+\Lambda a^{2}}
$$


e a temperatura como

$$
T_{h}=\frac{k_{h}}{2 \pi}=\frac{\Lambda \prod_{j \neq h}\left|r_{h}-r_{j}\right|}{4 \pi\left(1+\Lambda a^{2}\right)\left(r_{h}^{2}+a^{2}\right)}
$$

sendo $r_{j}$ os demais horizontes da geometria. Tal relação é dependente da gravidade superficial no horizonte, que também pode ser calculada para outros horizontes com a expressão acima, apenas mudando-se $r_{h}$ para o valor de $r$ neste horizonte. A capacidade térmica do buraco negro é dada por

$$
C=\frac{2 \pi k_{2} r_{h}}{\left.\left(1+\Lambda a^{2}\right) \frac{\partial k_{2}}{\partial r_{h}}\right|_{\Lambda, a, Q}}
$$

sendo $k_{2}$ a gravidade superficial do segundo horizonte (dos quatro possível em ordem crescente em $r$ ), dada por

$$
k_{2}=\frac{\Lambda}{2\left(r_{2}^{2}+a^{2}\right)\left(1+\Lambda a^{2}\right)}\left[3 r_{2}^{2}-r_{2} \frac{1-\Lambda a^{2}}{\Lambda}+\frac{a^{2}+Q^{2}}{\Lambda r_{2}}\right] \text {. }
$$

O fato peculiar desta expressão para $C$ é que o denominador pode, com a escolha adequada dos parâmetros do buraco negro, ter valor nulo. Tal descontinuidade da função $C$ representa uma transição de fase de segunda ordem [62]. Para o buraco negro de Reissner-Nordström em específico, $a=$ $\Lambda=0$ e temos que

$$
C_{R N}=\frac{4 M T S^{3}}{\pi Q^{4}-4 T^{2} S^{3}}=\frac{2 \pi \sqrt{M^{2}-Q^{2}}\left[-2 M^{2}+Q^{2}-2 M \sqrt{M^{2}-Q^{2}}\right]^{2}}{2 M^{3}-3 M Q^{2}+2\left(M^{2}-Q^{2}\right)^{3 / 2}},
$$

que diverge para

$$
Q_{t f}=\frac{\sqrt{3}}{2} M
$$

Este é ponto em que a capacidade térmica torna-se singular, e muda de sinal de positiva para negativa, associado portanto a uma mudança de fase de segunda ordem. A proposição inicial é de que os modos quasi-normais 


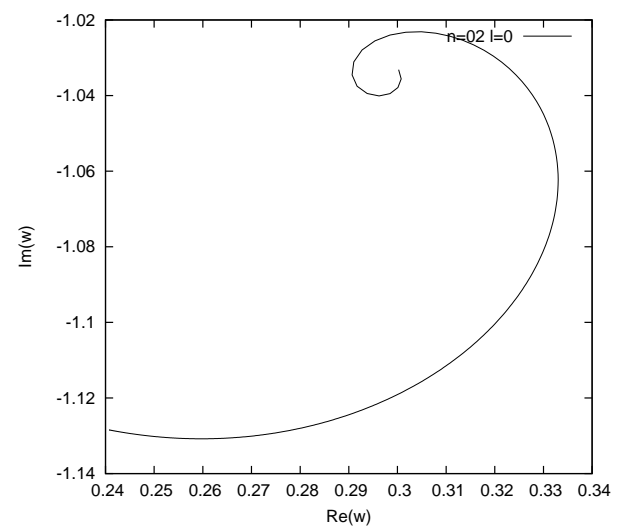

Figura 3.10: Campo de Dirac com $j=1 / 2, n=2$. Gráfico ' $\omega$ ', aparecimento do comportamento oscilatório.

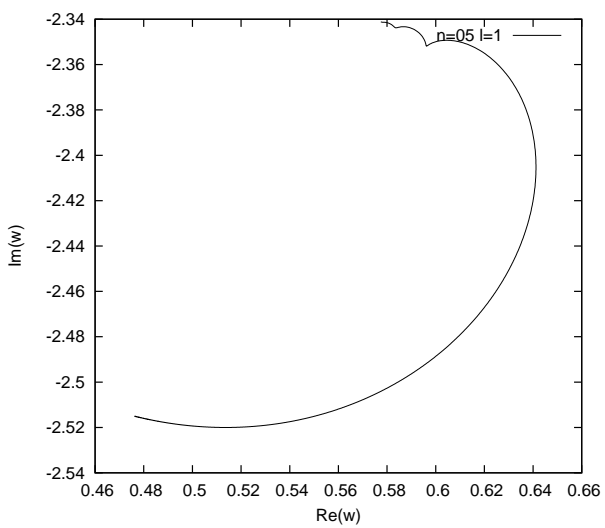

Figura 3.11: Campo de Dirac com $j=3 / 2, n=5$. Gráfico ' $\omega$ ' aparecimento do comportamento oscilatório

carreguem algum tipo de informação termodinâmica associado a essa descontinuidade [49], uma vez que $\frac{d \omega_{I}}{d \omega_{R}}$ diverge, da mesma maneira que $C$, para determinados valores de $l, n$ e $Q$.

As curvas dos modos quasi-normais para a geometria de Reissner-Nordström do tipo $\left(\omega_{R} \times \omega_{I}\right),\left(\omega_{R} \times Q\right)$ e $\left(\omega_{I} \times Q\right)^{7}$ têm um formato bastante peculiar. Os gráficos do tipo $\left(\omega_{R} \times \omega_{I}\right)$ tomam a forma de espiral, para altos valores de $n$ e baixos de $l[43,44]$, e os demais um formato oscilatório como uma função da carga.

A característica peculiar aqui observada é a de que quando o comportamento do gráfico $\omega$ começa a exibir o formato espiral, teremos $\hat{D} \equiv \frac{d \omega_{I}}{d \omega_{R}} \rightarrow$ $C_{R N} \rightarrow \infty$, para as perturbações de spin meio e escalares [49], para os primeiros valores de $l$.

A proposta é a de verificar se tal comportamento se mantem para valores diferentes de $l$, o que tornaria a 'conjectura' robusta, e mais especificamente obter o mesmo comportamente para perturbações gravitacionais, o que seria uma indicação forte de que de fato os modos quasi-normais carregam alguma propriedade termodinâmica ainda não elucidada, uma vez que, fisicamente, há mais sentido falar em perturbações gravitacionais do que em propagação

\footnotetext{
${ }^{7}$ A tais gráficos designaremos por simplicidade como $\omega, R$ e $I$, respectivamente, daqui por diante.
} 
de campos testes em dadas geometrias ${ }^{8}$.

Para as perturbações de spin meio e escalar, obtivemos resultados que confirmam a conjectura como proposta em [49]. Os gráficos das figuras (3.103.15), confirmam o comportamento apontado acima.
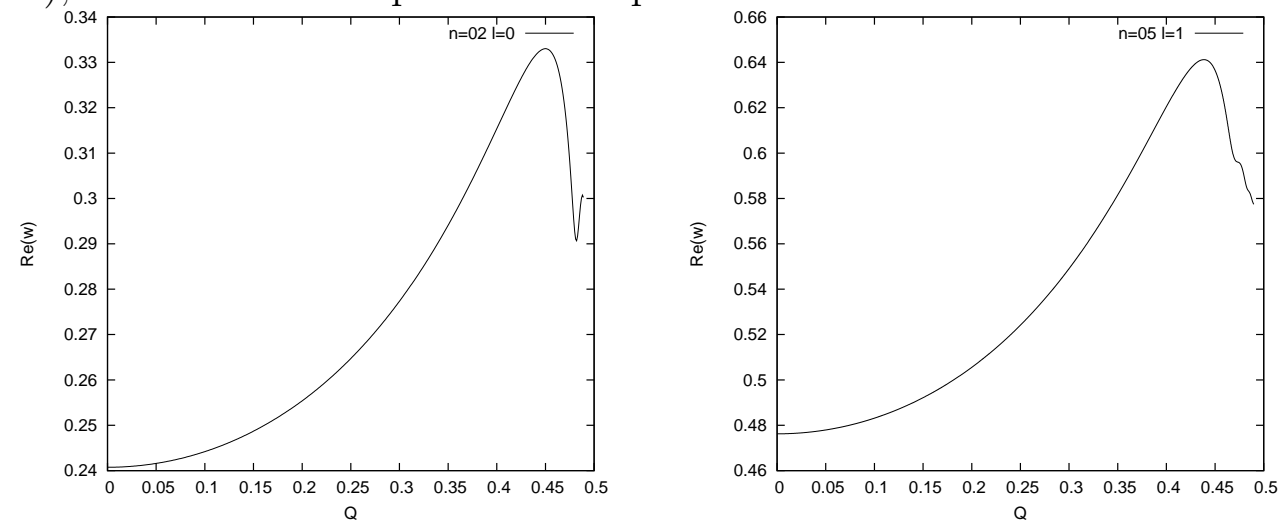

Figura 3.12: Campo de Dirac com Figura 3.13: Campo de Dirac com $j=1 / 2, n=2$. Gráfico ' $R$ ', apa- $j=3 / 2, n=5$. Gráfico ' $R$ ', aparecimento de um pico relacionado à recimento de um pico relacionado à transição de fase. transição de fase.
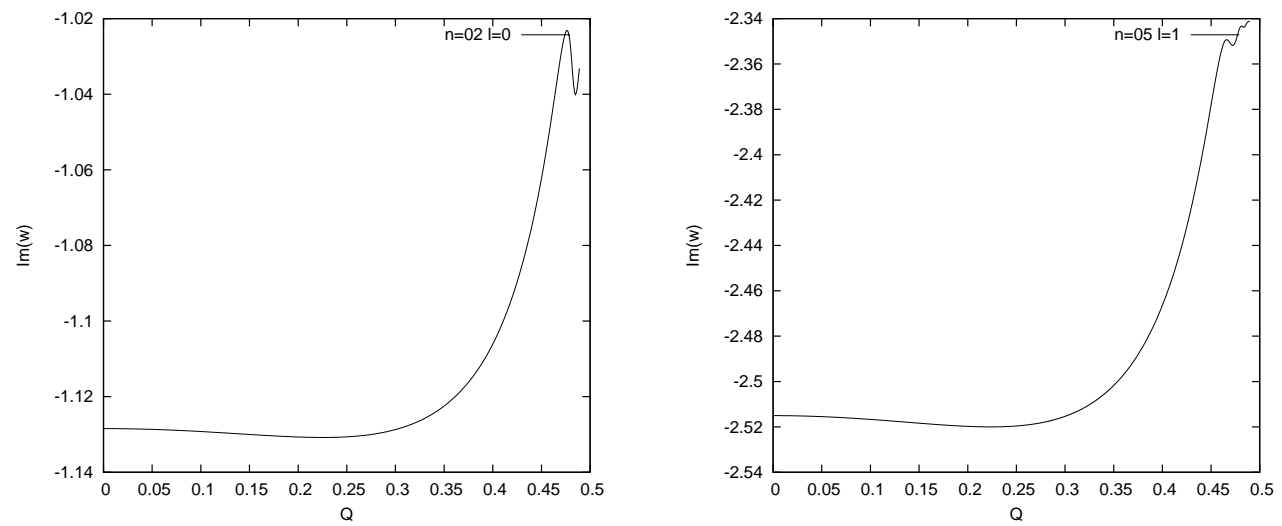

Figura 3.14: Campo de Dirac com Figura 3.15: Campo de Dirac com $j=1 / 2, n=2$. Gráfico ' $I$ '. $j=1 / 2, n=2$. Gráfico ' $I$ '.

A condição de que o gráfico $\omega$ adquira um formato espiral depende do valor de momento angular considerado. Quanto maior $l$ (ou mesmo $j$ ), maior

\footnotetext{
${ }^{8}$ Pois por certo estes campos perturbam a ada geometria também.
} 
é o valor de $n$ para o qual $\omega$ tem este formato. Nomeamos $n_{c}$ o valor de $n$, a partir do qual, para um dado $l$, o gráfico $\omega$ toma um formato espiral. Então, pelos resultados obtidos (e em acordo com [49]), para a perturbação de spin $1 / 2, n_{c}=2,5$ para $l=0,1$ ou $j=1 / 2,3 / 2$.

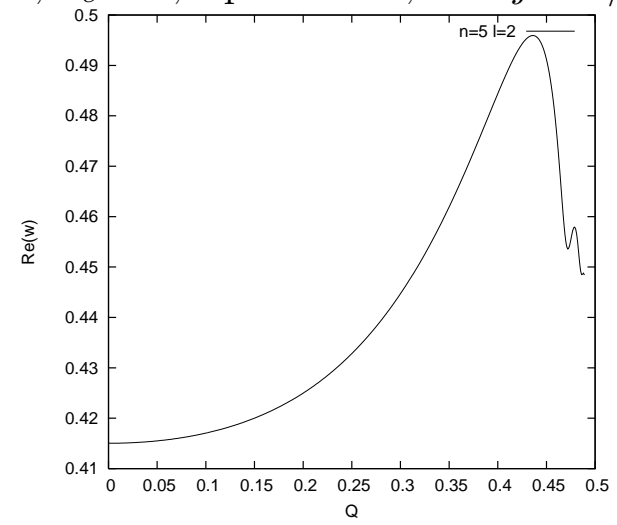

Figura 3.16: Modos quasi-normais, perturbação gravitacional.

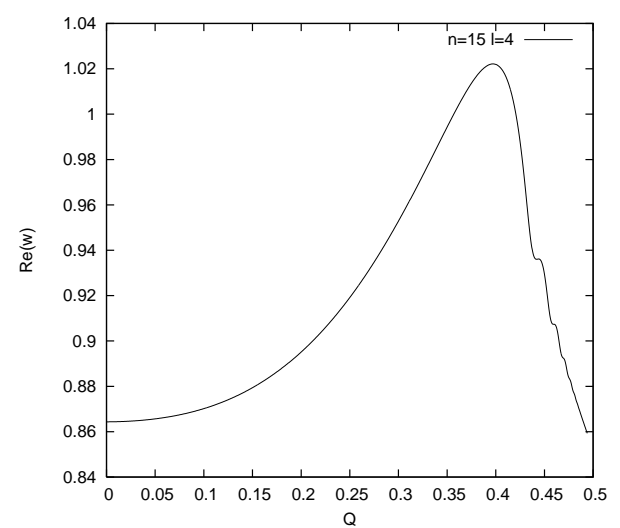

Figura 3.18: Modos quasi-normais, Figura 3.19: Modos quasi-normais, perturbação gravitacional.

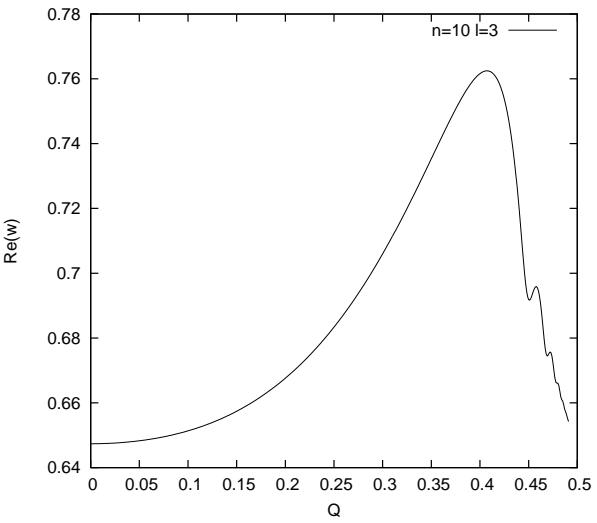

Figura 3.17: Modos quasi-normais, perturbação gravitacional.

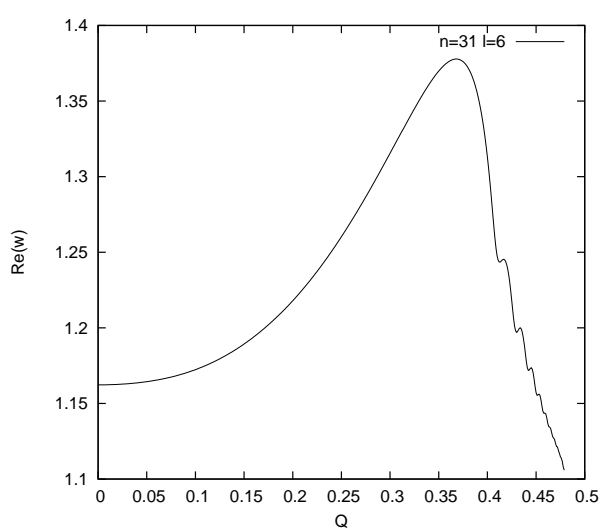

Em todos os gráficos para $n>n_{c}$, o comportamento espiral se acentua, e ainda quanto maior $n$, mais próximo $\omega$ estará de uma espiral concêntrica, 
Tabela 3.1: Valores de $Q_{d}$ para diferentes momentos angulares.

\begin{tabular}{|c|c|c|c|c|c|}
\hline$l$ & 2 & 3 & 4 & 5 & 6 \\
\hline$n_{c}$ & 5 & 10 & 15 & 22 & 31 \\
\hline$Q_{0}$ & 0.436 & 0.407 & 0.397 & 0.383 & 0.368 \\
\hline
\end{tabular}

como o esperado por [41]. Também a oscilação dos gráficos $R$ e $I$ torna-se mais acentuada conforme o valor de $n$ aumenta.

O comportamento evidenciado é a proposta de que exatamente no ponto de transiçãode fase, $Q_{t f}$, os modos quasi-normais sofrem uma descontinuidade semelhante em $\hat{D}$. Tal evidencia se sustem para perturbações calculadas em outros valores de $l$, e ainda para a propagação do campo escalar.

A perturbação gravitacional, contudo, não evidencia tal efeito, e ao contrário, denota este fato como sendo apenas uma possível coincidência numérica uma vez que os gráficos não apresentam um corpontamento robusto, como o sugerido em [49].

Os resultados são qualitativamente semelhantes aos da propagação de campos na geometria: os gráficos $\omega$ oscilam em formato espiral, e os gráficos $I$ e $R$ oscilam em função da carga, para valores altos de $n$. Entretanto o ponto em que esta oscilação começa a acontecer é diferente para os diferentes valores de $l$ e $n_{c}$ na perturbação gravitacional, e mais imporante, não coincide com $Q_{t f}$. Os plots para a perturbação gravitacional, com diferentes valores de $l$, seguem nas figuras (3.16-3.23).

Destes gráficos, podemos inferir que o ponto em que $\hat{D}$ diverge não coincide com o ponto em que $C$ diverge, ou seja, $\left.\omega_{R}(\max )\right|_{Q_{d}}$ tem $Q_{d} \neq Q_{t f}$. Também ao plotarmos $\hat{D}$ em função da carga, este gráfico tem um formato essencialmente diferente do gráfico $C \times Q$, como acontece para o campo de spin meio quando para um dado $l, n=n_{c}$.

Os valores de $Q_{d}$, para os quais $\omega_{R}$ atinge o máximo são listados na tabela 3.1. 


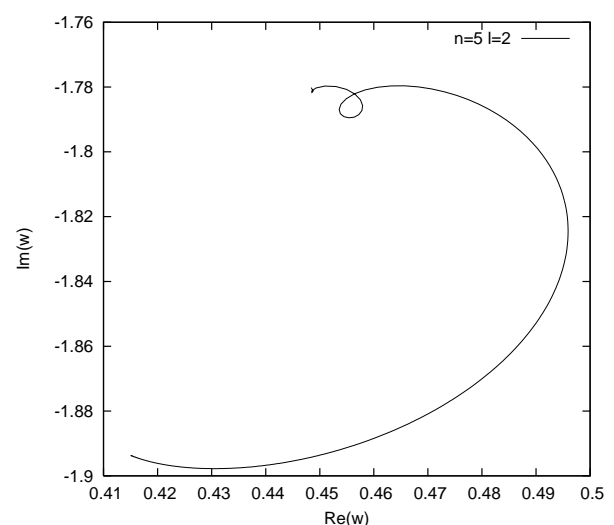

Figura 3.20: Modos quasi-normais, perturbação gravitacional.

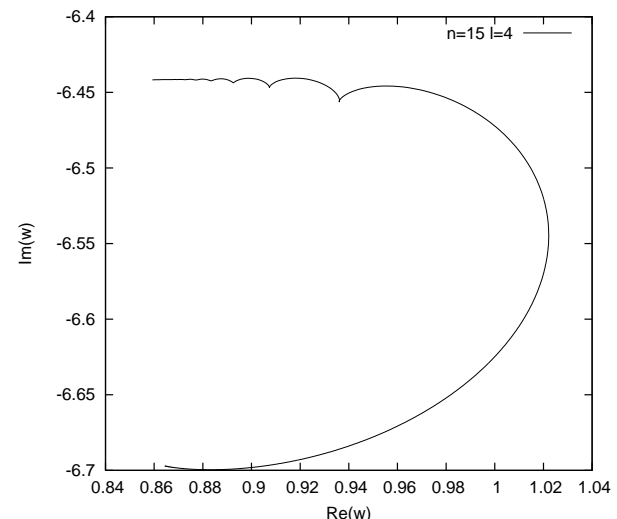

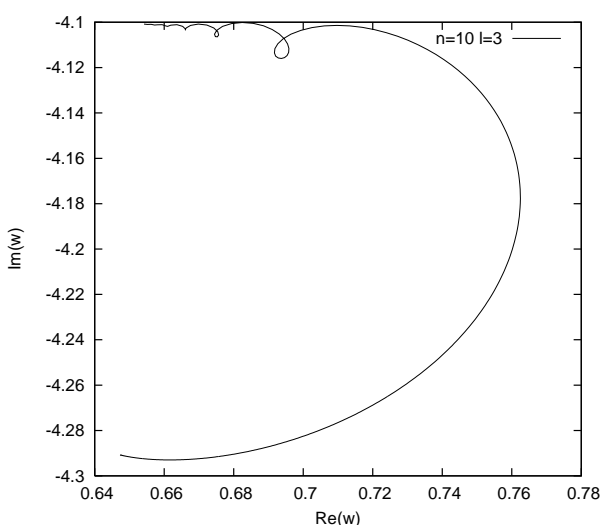

Figura 3.21: Modos quasi-normais, perturbação gravitacional.

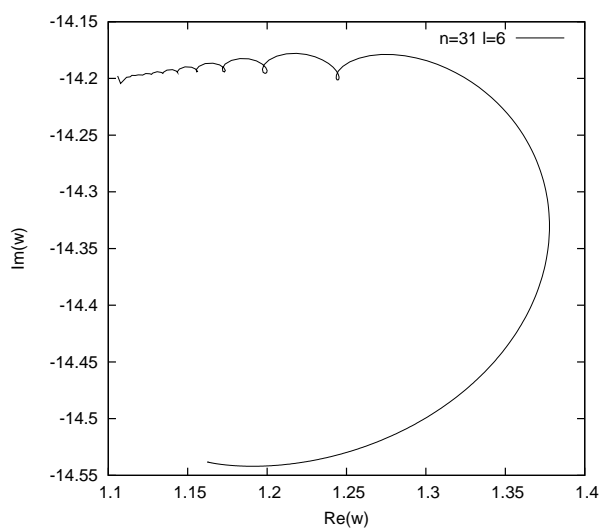

Figura 3.22: Modos quasi-normais, Figura 3.23: Modos quasi-normais, perturbação gravitacional. perturbação gravitacional. 
Tabela 3.2: Valores de $Q_{d}$ para diferentes momentos angulares.

\begin{tabular}{|c|c|c|c|c|c|c|c|c|}
\hline$l$ & 2 & 3 & 4 & 5 & 6 & 7 & 8 & 9 \\
\hline$Q_{0}$ & 0.4891 & 0.4860 & 0.4841 & 0.4828 & 0.4819 & 0.4812 & 0.4807 & 0.4802 \\
\hline$l$ & 10 & 11 & 12 & 13 & 14 & 15 & 16 & 17 \\
\hline$Q_{0}$ & 0.4799 & 0.4796 & 0.4794 & 0.4792 & 0.4790 & 0.4788 & 0.4787 & 0.4787 \\
\hline
\end{tabular}

\subsubsection{O Comportamento de $\omega_{0}$}

Embora os resultados da perturbação gravitacional não confirmem a conjectura de que os modos quasi-normais levam alguma informação da termodinâmica de buracos negros, relacionados à mudança de fase de segunda ordem para estas geometrias, um comportamente peculiar emerge quando investigamos os gráficos do tipo $\omega_{0} \times Q,{ }^{9}$ em que $\omega_{0}$ é a frequência relacionada com a quantização da área da seção 3.2.

Para tais tipos de gráfico, o pico de $\omega_{0}$ acontece próximo a $Q \sim 0.48$ sempre que $l=n$ (nomearemos o ponto em que $\omega_{0}$ atinge seu valor máximo de $\left.Q=Q_{0}\right)$. Isto desconfigura uma possível relação com a transição de segunda ordem, visto que tal valor está $10.5 \%$ distante de $Q_{t f}$, entretanto revela um comportamento interessante: sempre que investigamos $l=n$, para valores cada vez maiores de $l$, obteremos que $\frac{d Q}{d \omega_{0}}$ diverge quando $Q_{0} \rightarrow 0.4787$. Uma tabela com os primeiros valores de $l$ é escrita em 3.2.

Quanto maior o valor de $l$, mais próximo o valor do primeiro pico na função $\omega_{0}$ fica de $Q_{0}=0.4787$.

Outro grupo de gráficos que segue este comportamento é a série $l=n-1$. Quanto maior o valor de $l$, mais próximo o valor do primeiro pico em $R I$ se aproxima de $Q_{0}$, de maneira que o ponto $Q_{0}$ parece se tratar de um 'ponto de acumulação' para estas duas séries. Tal comportamento acontece também para a série $l=n-2$, embora se perca precisão, para se saber com certeza o valor do primeiro pico, $Q_{p}$, com 4 casa após a vírgula, o que impossibilita o estudo da série $l=n-3$. Um plot genérico de $l=n$ para o gráfico $R I$

\footnotetext{
${ }^{9}$ Nomearemos tais gráficos de gráficos $R I$
} 
segue na figura (3.24): quanto maior o valor de $l$, maior a proximidade do pico com o valor de acumulação $Q_{0}$.

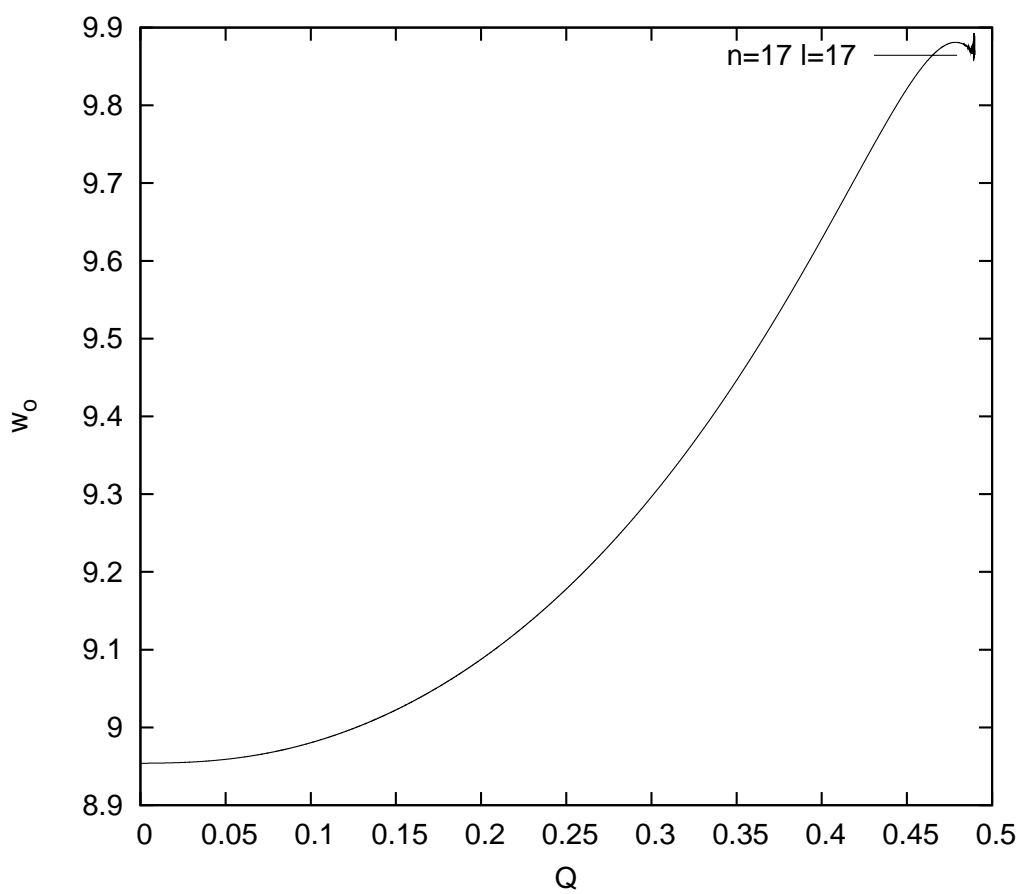

Figura 3.24: $\omega_{0}$ como função de $Q$, para a série $l=n$ : o pico situa-se em $Q_{0}=0.4787$. 


\section{Capítulo 4}

\section{Modos Quasi-Normais em Buracos Negros Cosmológicos}

Temos visto nos últimos anos um crescente interesse pelo estudo de buracos negros com constante cosmológica e sua associação com a conjectura AdSCFT. A análise de perturbações em tais tipos de buracos negros é, de maneira geral, bastante similar ao de buracos negros sem constante cosmológica. Notadamente, embora o espaço-tempo não seja assintóticamente o de Minkowski (sendo portanto não-trivial, por exemplo, definições como a de energia de partículas medidas por observadores externos ao horizonte de eventos), existem equações de perturbação destas métricas com simetria esférica, visto que, o espaço-tempo pode ser escrito em uma variedade $V^{2} \times S^{D-2}$.

Os buracos negros de de Sitter e anti-de Sitter diferem apenas pelo termo de constante cosmológica ser negativo ou positivo, respectivamente. O elemento de linha de tais tipos de objetos advem de uma métrica genérica para buracos negros com simetria esférica dada por [63]

$$
d s^{2}=g_{a b} d x^{a} d x^{b}+r^{2} d \Omega_{D-2}^{2}
$$

Aqui $D$ é o número de dimensões do espaço-tempo e $\Omega_{D-2}$ a esfera- $(D-2)$, cuja métrica é dada por $d \Omega_{D-2}^{2}=\gamma_{i j} d z^{j} d z^{i}$. O tensor de curvatura de Ricci 
é dado por

$$
R_{i j}=(D-3) K \gamma_{i j}
$$

em que $K$ é a curvatura intrínsica da sub-variedade $S$. Por sua vez, os índices $a$ e $b$ tem seu escopo limitado à sub-variedade bidimensional $V^{2}$.

Quando da presença de uma constante cosmológica, vale para $g$ que

$$
\lim _{r \rightarrow \infty} g_{a b} \neq 0
$$

de maneira que o escalar de Ricci é proporcional a esta constante de acordo com as equações de Einstein.

Neste capítulo iremos trabalhar essencialmente com buracos negros definidos por um conjunto de 4 parâmetros: massa, carga, constante cosmológica e dimensão do espaço-tempo

Cabe a ressalva de que embora não haja evidência física (experimental) para que as equações de Einstein permaneçam as mesmas em mais do que 4 dimensões, há boas motivações teóricas para o seu estudo fornecidas pela conjectura da correspondência AdS-CFT.

O elemento de linha da solução de buraco negro com estes 4 parâmetros lê-se

$$
\begin{gathered}
d s^{2}=-\left[1-\frac{2 M}{r^{D-3}}+\frac{Q^{2}}{r^{2 D-6}}-\Lambda r^{2}\right] d t^{2} \\
+\left[1-\frac{2 M}{r^{D-3}}+\frac{Q^{2}}{r^{2 D-6}}-\Lambda r^{2}\right]^{-1} d r^{2}+r^{2} d \Omega_{D-2}^{2}
\end{gathered}
$$

Como já descrito em capítulos anteriores, uma perturbação gravitacional neste elemento de linha corresponde à adição de um tensor $h$ em primeira ordem quando consideramos $g$ de ordem zero. Dada a simetria esférica do problema, podemos decompor este tensor $h$ em componentes escalares $h_{a b}$, componentes vetoriais $h_{i a}$ e componentes tensoriais $h_{i j}$. De acordo com esta classificação, podemos escrever cada componente de $h$ de maneira específica, 
preservando com isto a simetria desejada. Temos

$$
\begin{aligned}
h_{a i} & =\nabla_{i} h_{a}+\tilde{h}_{a i}, \quad \nabla^{i} \tilde{h}_{a i}=0, \\
h_{i j} & =h_{L} \gamma_{i j}+h_{T i j}, \quad \gamma^{k i} h_{T i j}=0, \\
h_{T i j} & =\left[\nabla_{i} \nabla_{j}-(D-2)^{-1} \gamma_{i j} \nabla^{k} \nabla_{k}\right] h_{T}+2 \nabla_{(i} h_{T j)}+\tilde{h}_{T i j},
\end{aligned}
$$

com $\nabla^{j} h_{T j}=0$ e $\nabla^{j} \tilde{h}_{T i j}=0$ e $\nabla$ representa a derivada covariante relativa a uma das duas sub-variedades. Com esta decomposição, podemos separar todas as componentes de $h$ em escalares $\left(h_{a b}, h_{a}, h_{L}, h_{T}\right)$, vetores $\left(\tilde{h}_{a i}, h_{T i}\right)$ ou tensores $\left(\tilde{h}_{T i j}\right)$, (todos em relação a $\left.S^{D-2}\right)$. Tal fenômeno ocorre pelo fato de que $\gamma$ é o único tensor não trivial em um espaço-tempo maximalmente simétrico em $S^{2}[66]$.

É possível tratar a perturbação, com estas decomposições, de maneira que tenhamos três escolhas para $h$ bem-definidas como já mencionado. Analisaremos em seguida a primeira destas escolhas para $h$, a saber, a perturbação tensorial. Tal perturbação não tem significação física em 4 dimensões, sendo puramente gauge. (Em 4 dimensões, por exemplo, ao considerarmos perturbações em um buraco negro de Schwarzschild, não há nenhum gauge possível cujas componentes de $h$ sejam apenas aquelas que se transformem tensorialmente em $S^{2}$. Isto que dizer que, devemos ter ou $h_{a b}$ ou $h_{a i}$ - ou ambos - não nulos para que de fato haja uma perturbação).

\subsection{Perturbações Gravitacionais Tensoriais}

Como a perturbação do campo eletromagnético não possui uma componente tensorial [63], os campos eletromagnéticos entram nas equações apenas como efeito direto na geometria de fundo. O tensor de perturbação da métrica, após considerado o gauge em termos de simetrias de vetores de Killing fica como

$$
h_{a b}=0, \quad h_{a i}=0, \quad h_{i j}=2 r^{2} H T_{i j},
$$


e de maneira semelhante, a perturbação do tensor momento-energia terá somente as componentes tensoriais:

$$
\delta T_{a b}=0, \quad \delta T_{a i}=0, \quad \delta T_{j}^{i}=\tau T_{j}^{i} .
$$

A equação de perturbação gravitacional é derivada considerando-se uma expansão nos termos da equação de Einstein até primeira ordem,

$$
\nabla^{a} \nabla_{a} H+\frac{D-2}{r} \nabla^{a} r \nabla_{a} H-\frac{£-2(D-3)}{r^{2}} H=-k^{2} \tau
$$

em que $£$ é o auto-valor da equação de Lichnerowicz, $\Delta T_{i j}=£ T_{i j}$ ou, $\Delta h_{i j} \rightarrow-\nabla^{k} \nabla_{k} h_{i j}-2 R_{i j k l} h^{k l}+2(D-3) h_{i j}$. No caso da simetria esférica especificamente, temos $£=l(l+D-3)$, sendo $l$ o número de multipolo de harmônico esférico. Então, fazendo-se uma mudança de variável no campo

$$
H \rightarrow r^{(2-D) / 2} \Phi
$$

obteremos a equação de perturbação em sua forma canônica,

$$
\left[\nabla^{a} \nabla_{a}+\frac{V(r)}{g_{t t}}\right] \Phi=-k^{2} r^{(D-2) / 2} \tau
$$

com $V(r)$ o potencial dado por

$$
\begin{array}{r}
V(r)=-\frac{g_{t t}}{r^{2}}\left[£-2(D-3)+\frac{(D-2) r}{2} \dot{f}+\frac{(D-2)(D-4)}{4} f\right] \\
=-\frac{g_{t t}}{r^{2}}\left[£+\frac{\left(D^{2}-14 D+32\right)}{4}-\frac{D(D-2)}{4} \Lambda r^{2}+\frac{(D-2)^{2}}{2 r^{D-3}} M\right. \\
\left.-\frac{(D-2)(3 D-8)}{4 r^{2 D-6}} Q^{2}\right]
\end{array}
$$

Para escrever a equação de perturbação em termos de um sistema de coordenadas do gênero $(r, t)$ - tipo espaço e tipo tempo, temos $\nabla^{2}=g^{a b}\left(\partial_{a} \partial_{b}-\right.$ $\left.\Gamma_{a b}^{c} \partial_{c}\right)$. Tendo em vista o elemento de linha (4.4), as únicas conexões não nulas são dadas por $\Gamma_{r r}^{r}=g^{r r} g_{r r, r} / 2$ e $\Gamma_{t t}^{r}=-g^{r r} g_{t t, r} / 2$, o que nos fornece 
para $\nabla^{2}$

$$
\nabla^{2}=g^{t t} \partial_{t}^{2}+\partial_{r}\left(g^{r r} \partial_{r}\right)
$$

A equação de perturbação neste caso se torna

$$
\left[g^{t t} \partial_{t}^{2}+\partial_{r}\left(g^{r r} \partial_{r}\right)+\frac{V(r)}{g_{t t}}\right] \Phi=0
$$

Queremos obter os modos quasi-normais de vibração do buraco negro e para tanto, como já se afirmou nos capítulos anteriores, muitos são os métodos disponíveis. Por uma questão de simplicidade, adotaremos aqui o método de integração em coordenadas nulas, como descrito pela primeira vez em [67]. Para encontrar a equação de perturbação em coordenadas nulas, devemos primeiramente definir tais coordenadas em termos da coordenada tartaruga, cuja representação é dada por $g_{t t} d r_{*}^{2}+g_{r r} d r=0$. Para o buraco negro de estudo, $d r_{*}=-g_{r r} d r$ e $\frac{\partial}{\partial r_{*}}=-g^{r r} \frac{\partial}{\partial r}$, do que $\frac{\partial^{2}}{\partial r_{*}^{2}}=g^{r r} \frac{\partial}{\partial r}\left(g^{r r} \frac{\partial}{\partial r}\right)$. Assim, a equação de perturbação com $r_{*}$ fica

$$
\left[-\frac{\partial^{2}}{\partial t^{2}}+\frac{\partial^{2}}{\partial_{r_{*}}^{2}}+\frac{V(r)}{g_{t t}}\right] \Phi=0 .
$$

As coordenadas nulas são definidas em termos de $t$ e de $r_{*} \operatorname{como} v=\frac{r_{*}+t}{2} \mathrm{e}$ $u=\frac{t-r_{*}}{2}$, de maneira que $\frac{\partial}{\partial v}=\frac{1}{2}\left[\frac{\partial}{\partial r_{*}}+\frac{\partial}{\partial t}\right]$ e $\frac{\partial}{\partial u}=\frac{1}{2}\left[-\frac{\partial}{\partial r_{*}}+\frac{\partial}{\partial t}\right]$, e com isto, obteremos a equação perturbativa em sua forma final para a integração com o formato

$$
\left[-4 \frac{\partial}{\partial u} \frac{\partial}{\partial v}+\frac{V(r)}{g_{t t}}\right] \Phi=0
$$

Na próxima sub-seção, definiremos o método utilizado, como em [67].

\subsubsection{Método de Integração em Coordenadas Nulas}

Na última equação temos representada uma perturbação tensorial em uma geometria de buracos negros com 4 parâmetros distintos. Queremos fazer a integração desta equação nas coordenadas $u$ e $v$. Trata-se de coordenadas 
nulas visto que o elemento de linha contendo $u$ e $v$ não tem os termos $g_{u u}$ e $g_{v v}$, ou seja, os vetores tangente à variedade $\partial_{v}$ e $\partial_{u}$ são vetores nulos designados por $<\partial_{u}, \partial_{u}>=<\partial_{v}, \partial_{v}>=0$.

A integração em uma grade de coordenadas $u$ e $v$ pode ser pensada de maneira usual ao discretizarmos os eixos em "pequenos" intervalos $\Delta u \mathrm{e}$ $\Delta v$ (o conceito de pequeno a que nos refirimos aqui está relacionado com o resultado a ser obtido pela integração. Uma vez que um dado perfil de campo é obtido, podemos diminuir a escala em uma ordem de grandeza, por exemplo, e verificar se este mesmo perfil se repete. Em caso positivo, temos então uma discretização pequena o suficiente para os propósitos do cálculo). Neste caso, as derivadas do campo podem ser expressas como variações $\Delta \Phi$ da seguinte maneira

$$
\begin{aligned}
\frac{\partial}{\partial v} \Phi & =\frac{\Delta \Phi}{\Delta v} \equiv \frac{\Phi_{E}-\Phi_{W}}{\Delta v} \\
\frac{\partial}{\partial u} \Phi & =\frac{\Delta \Phi}{\Delta u} \equiv \frac{\Phi_{N}-\Phi_{S}}{\Delta u} .
\end{aligned}
$$

Tal discretização do campo obedece a um diagrama em que $u$ ocupa o eixo das abcissas e $v$ o eixo das ordenadas, de maneira que uma variação no campo $\Phi$ possa ser escrita como o valor deste campo no seu ponto ao norte menos o valor do campo no ponto ao sul deste, para $u$, e de maneira semelhante para $v$ (variação de $\Phi$ em relação aos pontos leste e oeste).

A equação de perturbação pode ser escrita em termos das variáveis acima, com a discretização do espaço-tempo como

$$
\Phi_{N}=\Phi_{E}+\Phi_{W}-\Phi_{S}+\Delta u \Delta v \frac{V(r)}{g_{t t}} \frac{\Phi_{W}+\Phi_{E}}{8}
$$

O componente final desta prescrição para obtermos o comportamento do campo $\Phi$, que descreve uma perturbação gravitacional tensorial nos buracos negros Reissner-Nordström de Sitter (anti-de Sitter) é a condição de contorno utilizada ao integrarmos. Para obtermos uma figura completa de $\Phi$ na grade de integração $(u, v)$, e por consequência, sua evolução temporal, precisamos de "duas retas de condição de contorno", a saber, todos os valores que $\Phi$ 
assume ao longo de duas retas iniciais, $u=u_{0}$ e $v=v_{0}$, por exemplo. Em geral, podemos evoluir um pacote de onda Gaussiano em uma das direções como condição inicial para o campo, e uma constante em outra direção [68],

$$
\begin{aligned}
& \Phi\left(u_{0}, v\right)=\exp \left[-\frac{\left(v-v_{c}\right)^{2}}{2 \sigma^{2}}\right] \\
& \Phi\left(u, v_{0}\right)=\text { constante. }
\end{aligned}
$$

$\left(v_{c}\right.$ representa o centro deste pacote na coordenada $v$ e $\sigma$ o quão concentrado ao redor deste centro ele está).

Há outros métodos de refinamento melhores para a convergência da equação (4.15), contudo, para os nossos propósitos de calcular o primeiro overtone, em cada valor diferente de carga, constante cosmológica e momento angulas, o método como proposto representa uma convergência relativamente boa: com uma grade de 10 milhões de pontos, obtemos um resultado equivalente ao de uma grade de 150 milhões de pontos, o que representa a convergência do método para esta primeira quantidade de pontos, já.

\subsubsection{Método Prony}

Uma vez obtido o sinal quasi-normal do campo em função de $t$, podemos aplicar um segundo método numérico para a obtenção das frequências, denominado método de Prony.

O primeiro passo para a aplicação de tal método é a decomposição do campo em termos dos modos quasi-normais de vibração como uma função do tempo,

$$
\Phi(t) \simeq \sum_{j=1}^{p} C_{j} e^{-i \omega_{j} t}
$$

Supondo que a oscilação quasi-normal comece em um dado tempo $t_{0} \mathrm{e}$ termine em um tempo posterior, $t=N \Delta u$, com $N$ um inteiro que respeita a condição $N \geq 2 p-1$. Então é certo que para o campo acima, teremos em 
cada tempo $t=n h$

$$
x_{n} \equiv \Phi(n h)=\sum_{j=1}^{p} C_{j} e^{-i \omega_{j} n h}=\sum_{j=1}^{p} C_{j} z_{j}^{n}
$$

O método nos permite calcular $z_{n}$ como uma função dos pontos do perfil $x_{n}$, e a partir disso obter as frequências $\omega_{j}$. Com o intuito de fazer isto, definimos uma função polinomial $A$,

$$
A(z)=\prod_{k=1}^{p}\left(z-z_{j}\right)=\sum_{m=0}^{p} \alpha_{m} z^{p-m}
$$

Considerando que $A\left(z_{i}\right)=0$, temos

$\sum_{m=0}^{p} \alpha_{m} x_{n-m}=\sum_{m=0}^{p} \alpha_{m} \sum_{k=1}^{p} C_{k} z_{k}^{n-m}=\sum_{k=1}^{p} C_{k} z_{k}^{n-p} \sum_{m=0}^{p} \alpha_{m} z_{k}^{p-m}=\sum_{k=1}^{p} C_{k} z_{k}^{n-p} A\left(z_{k}\right)=0$.

com $\alpha_{0}=1$. Da equação anterior, podemos deduzir que $\sum_{m=1}^{p} \alpha_{m} x_{n-m}=$ $-x_{n}$. Substituindo os $N-p$ valores possíveis de $n$, teremos $N-p+1 \geq$ $p$ equações lineares para $p$ coeficientes $\alpha_{m}$ indeterminados. Expandindo a equação para $x$, teremos

$$
\left(\begin{array}{cccc}
x_{p-1} & x_{p-2} & \cdots & x_{0} \\
x_{p} & x_{p-1} & \cdots & x_{1} \\
\cdots & \cdots & \cdots & \cdots \\
x_{N-1} & x_{N-2} & \cdots & x_{N-p}
\end{array}\right)\left(\begin{array}{c}
\alpha_{1} \\
\alpha_{2} \\
\cdots \\
\alpha_{p}
\end{array}\right)=-\left(\begin{array}{c}
x_{p} \\
x_{p+1} \\
\cdots \\
x_{N}
\end{array}\right)
$$

Renomeando a equação acima como $X \alpha=-x$, podemos resolvê-la como

$$
\alpha=-\left(X^{\dagger} X\right)^{-1} X^{\dagger} x
$$

(devemos aplicar o operador $X^{\dagger}$ na equação visto que a matriz $X$ não é quadrada, após o que poderemos tomar sua inversa).

Depois de calculados os coeficientes $\alpha_{m}$ na equação acima, poderemos 
encontrar $z_{k}$ pelos zeros de (4.22) e através destes as frequências,

$$
\omega_{j}=\frac{i}{\Delta v} \ln z_{j}
$$

\subsection{Análise de Horizontes}

Os horizontes dos buracos negros com constante cosmológica definidos pelo elemento de linha (4.4) têm lugar onde a função radial $\left(g^{r r} \equiv f(r)\right)$ é nula. O fato de podermos considerar os horizontes como o zero desta função está conectado com a simetria esférica da métrica: para elementos de linha que não tenham simetria esférica, $f(r)=0$ não representa os horizontes de evento.

Além do horizonte de eventos, na presença de carga e constante cosmológica espera-se a ocorrência ainda de um segundo horizonte de eventos interno (horizonte de Cauchy) e de um horizonte externo, devido à constante cosmológica, denominado horizonte de de Sitter.

Por questões de simplicidade, adotaremos que o horizonte de eventos tem lugar em $r_{h}=1$. Isto corresponde a fixar o valor da massa em termos da carga e da constante cosmológica em

$$
M=\frac{1-\Lambda+Q^{2}}{2}
$$

uma vez que $f\left(r_{h}\right)=0$. Um caso de buracos negros extremos para esta função ocorre sempre que um segundo horizonte $r_{C}$ tenha $r_{C}=1$. Isto representa uma segunda restrição que relaciona a constante cosmológica com a carga da solução. Para obter esta relação, podemos considerar caso a caso, a partir de $D=5$ dimensões. Sendo $r_{h}=r_{C}=1$, além da condição (4.27) sobre a massa é necessário ainda que $2-3 \Lambda-2 M=0$. Para 6 dimensões esta condição extra é dada por $3-4 \Lambda-4 M=0$ e em 7 dimenões por $4-5 \Lambda-6 M=0$. A prescrição genérica para um número qualquer de dimensões (maior do que 4) fica

$$
1-2 \Lambda-(D-4) Q^{2}=0
$$


Desta maneira, para cada solução com uma dada constante cosmológica, há um valor de carga,

$$
Q_{c}=\sqrt{\frac{1-2 \Lambda}{D-4}}
$$

que representa a coalescência de dois horizontes em $r=1$. A presença de modos instáveis para valores de $Q$ próximos aos valores extremos acima foi verificado para o potencial escalar em $[6,7]$.

Dentre as demais raízes, para os diversos valores possíveis de $\Lambda$, teremos sempre uma terceira raiz positiva de $f(r)$ que representa o horizonte cosmológico, $r_{S}$, com as outras negativas. Para determinar ainda se o caso extremo acima representa a coalescência do horizonte de Cauchy ou cosmológico, é necessário saber o número de dimensões do espaço-tempo. Para $D=5$, por exemplo, as raízes de $f(r)$ estão sempre em pares $\pm a$, e, além de \pm 1 representarem raízes duplas no caso extremo, teremos mais duas raízes dadas por $r_{S}= \pm\left(\frac{1}{\Lambda}-2\right)$. Isto indica que há uma coalizão do horizonte cosmológico com o horizonte de eventos no caso em que $\Lambda<1 / 3$, e dos dois horizontes de eventos, em caso contrário, $\Lambda>1 / 3$. Para $\Lambda=1 / 3$, os três horizontes estão localizados em $r=1$.

É praticamente impossível determinar os zeros da função $f(r)$ analiticamente para um número arbitrário de dimensões, e sem informações extras a respeito da carga e da constante cosmológica, uma vez que a equação tem número de raízes proporcional à dimensão do espaço-tempo. Para analisarmos a presença de horizontes e nas próximas seções os modos quasinormais, consideraremos um espaço-tempo de 5 dimensões, com função radial $f(r)=1-\frac{2 M}{r^{2}}+\frac{Q^{2}}{r^{4}}-\Lambda r^{2}$. Neste caso, os horizontes são as raízes da equação

$$
-\Lambda r^{6}+r^{4}-2 M r^{2}+Q^{2}=0
$$

que pode ser simplificada a uma equação de terceira ordem com a substituição $r^{2}=x$. Se uma das raízes da equação for fixada em 1, automaticamente, uma segunda raiz será -1, uma vez que as raízes ocorrem em 'duplas $\pm a$ '. 
Com a substituição de $x$ em termos de $r$, podemos calcular analiticamente as demais raízes do polinômio restante. Temos

$$
-\Lambda r^{6}+r^{4}-2 M r^{2}+Q^{2}=\left[-\Lambda x^{2}+(1-\Lambda) x-Q^{2}\right](x-1)
$$

e com isto, os demais horizontes ocorrerão em

$$
\begin{aligned}
& r_{1}=\sqrt{\frac{1-\Lambda+\sqrt{\Lambda^{2}-2 \Lambda+1-4 \Lambda Q^{2}}}{2 \Lambda}} \\
& r_{2}=\sqrt{\frac{1-\Lambda-\sqrt{\Lambda^{2}-2 \Lambda+1-4 \Lambda Q^{2}}}{2 \Lambda}} .
\end{aligned}
$$
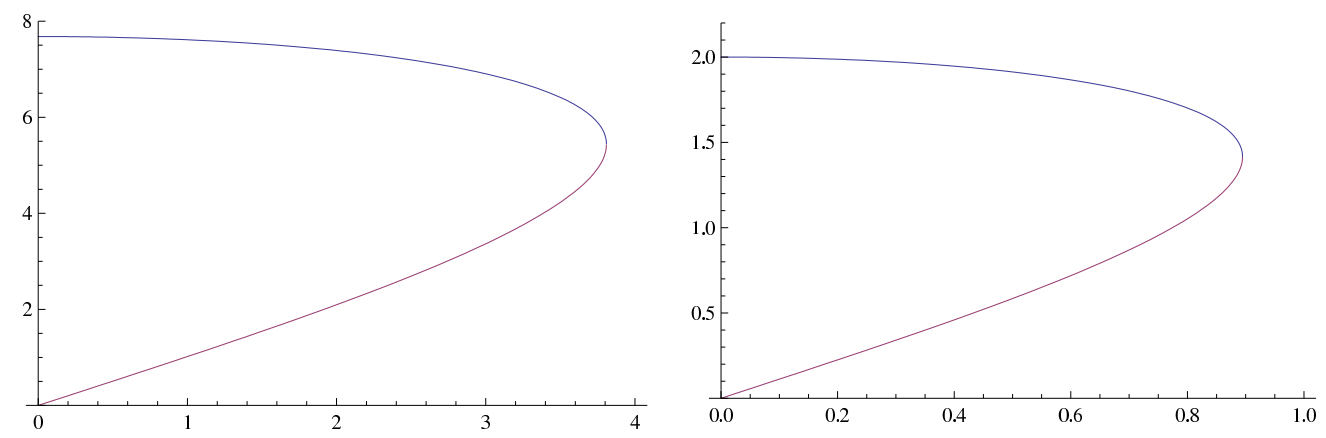

Figura 4.1: Horizontes de um buraco negro de Sitter com carga em 5 dimensões e $\Lambda=0.033$.

Figura 4.2: Horizontes de um buraco negro de Sitter com carga em 5 dimensões e $\Lambda=0.2$

Tomando dois diferentes valores de constante cosmológica, fazemos um gráfico das raízes nas figuras 4.1 e 4.2 .

Nestas figuras é possível perceber que, embora a situação em que $r=1$ é uma raiz dupla represente um buraco negro extremo, poderemos ter valores maiores de $Q$, contanto que um dos horizontes seja mantido em $r=1$. Neste caso não é mais o horizonte de eventos que se situa neste ponto, mas o de Cauchy. Uma situação extrema, em que os horizontes cosmológicos e de eventos coalescem ao mesmo ponto $r_{h}=r_{S}=1$ pode ser observada ainda na figura (4.3), caso em que o horizonte de Cauchy tem $r_{C}<1$, e $r=1$ passa a representar o horizonte cosmológico para qualquer valor de carga. 


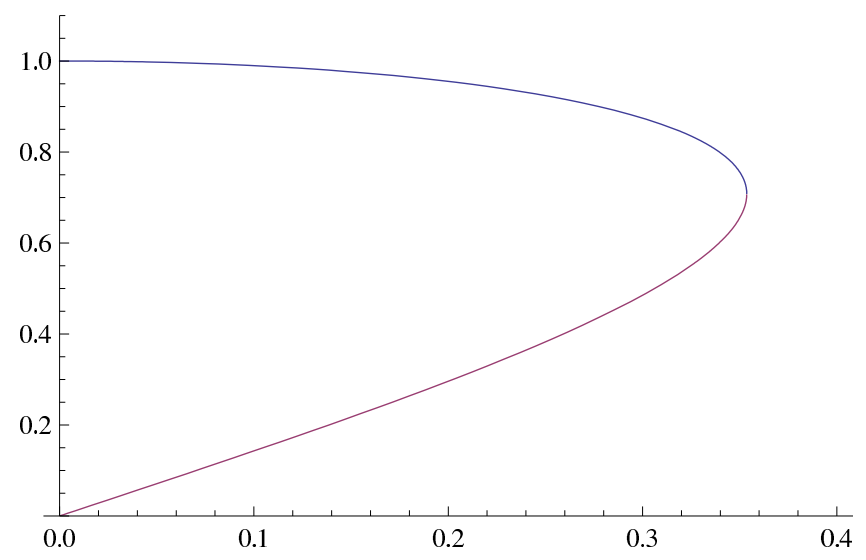

Figura 4.3: Horizontes de um buraco negro de Sitter com carga em 5 dimensões e $\Lambda=0.5$. O horizonte cosmológico passa a se situar em $r_{h}=1$, e os horizontes de eventos estão representados pelas linhas do gráfico.

Na próxima seção exploraremos os resultados obtidos para a perturbação gravitacional tensorial em 5 dimensões na solução com constante cosmológica positiva, para o que é de fundamental importância a análise da posição dos horizontes de eventos ou cosmológico.

\subsection{Modos Quasi-normais com a Perturbação Tensorial}

Através do método de integração de coordenadas nulas e com o auxílio do método Prony, obtemos o perfil do campo gravitacional sobre a atuação um potencial tensorial [64]. Para $\Lambda=0.0167$, as frequências quasi-normais estão listadas na tabela 4.1. Para $D=4$, tanto o potencial tensorial, como o vetorial representam configurações de gauge puro, sem modos dinâmicos, portanto, já tendo sido estudados a propagação de um campo escalar em tal geometria [65].

Há duas características peculiares aos modos de vibrações do buraco negro em um Universo de Sitter com um termo pequeno de constante cosmológica: quanto maior a carga do buraco negro, menor a frequência e a atenuação da oscilação do buraco negro. Pode-se dizer que quando a carga se aproxima 
Tabela 4.1: Modos quasi-normais para um buraco negro com $\Lambda=1 / 60$ e $l=2$.

\begin{tabular}{|c|c|c|c|c|c|}
\hline$Q$ & $\omega_{R}$ & $\omega_{I}$ & $Q$ & $\omega_{R}$ & $\omega_{I}$ \\
\hline 0.1 & 1.107 & 0.337 & 0.2 & 1.099 & 0.336 \\
\hline 0.3 & 1.081 & 0.335 & 0.4 & 1.055 & 0.325 \\
\hline 0.5 & 1.021 & 0.307 & 0.6 & 0.984 & 0.284 \\
\hline 0.7 & 0.945 & 0.261 & 0.8 & 0.902 & 0.141 \\
\hline 0.9 & 0.858 & 0.220 & 1.0 & 1.023 & 0.0648 \\
\hline 1.1 & 0.762 & 0.209 & 1.2 & 0.715 & 0.195 \\
\hline 1.3 & 0.673 & 0.186 & 1.4 & 0.631 & 0.179 \\
\hline 1.5 & 0.592 & 0.171 & 1.6 & 0.554 & 0.164 \\
\hline 1.7 & 0.519 & 0.156 & 1.8 & 0.486 & 0.149 \\
\hline 1.9 & 0.455 & 0.142 & 2.0 & 0.426 & 0.135 \\
\hline 2.1 & 0.398 & 0.129 & 2.2 & 0.372 & 0.122 \\
\hline 2.3 & 0.347 & 0.115 & 2.4 & 0.323 & 0.109 \\
\hline 2.5 & 0.301 & 0.103 & 2.6 & 0.279 & 0.0963 \\
\hline 2.7 & 0.259 & 0.0907 & 2.8 & 0.239 & 0.0845 \\
\hline 2.9 & 0.220 & 0.0791 & 3.0 & 0.200 & 0.0738 \\
\hline 3.1 & 0.180 & 0.0678 & 3.2 & 0.160 & 0.0606 \\
\hline 3.3 & 0.140 & 0.0517 & 3.4 & 0.123 & 0.0413 \\
\hline 3.5 & 0.108 & 0.0312 & 3.6 & 0.0940 & 0.0252 \\
\hline 3.7 & 0.0603 & 0.0274 & 3.8 & 0.0109 & 0.00859 \\
\hline
\end{tabular}


de seu valor extremo, o que corresponde à coalizão dos horizontes de evento e cosmológico, a frequência natural de oscilação, $\omega_{0}=\sqrt{\omega_{R}^{2}+\omega_{I}^{2}}$, tende a zero. O valor máximo de carga é dado por

$$
Q_{\max }=\frac{1-\Lambda}{2 \sqrt{\Lambda}}
$$

o que corresponde a $r_{1}=r_{2}$. Para o caso específico em que $\Lambda=1 / 60$, temos $Q_{\max } \sim 3,808$, de maneira que o último valor calculado expresso na tabela é 99,8\% do valor total máximo que $Q$ pode alcançar (e por este motivo tem $\left.\omega_{0} \sim 0\right)$.

Há contudo, ainda, o ponto de destaque $Q_{c}=\sqrt{\frac{1-2 \Lambda}{D-4}}$, calculado na seção anterior, que corresponde à coalizão entre os horizontes de Cauchy e de eventos.

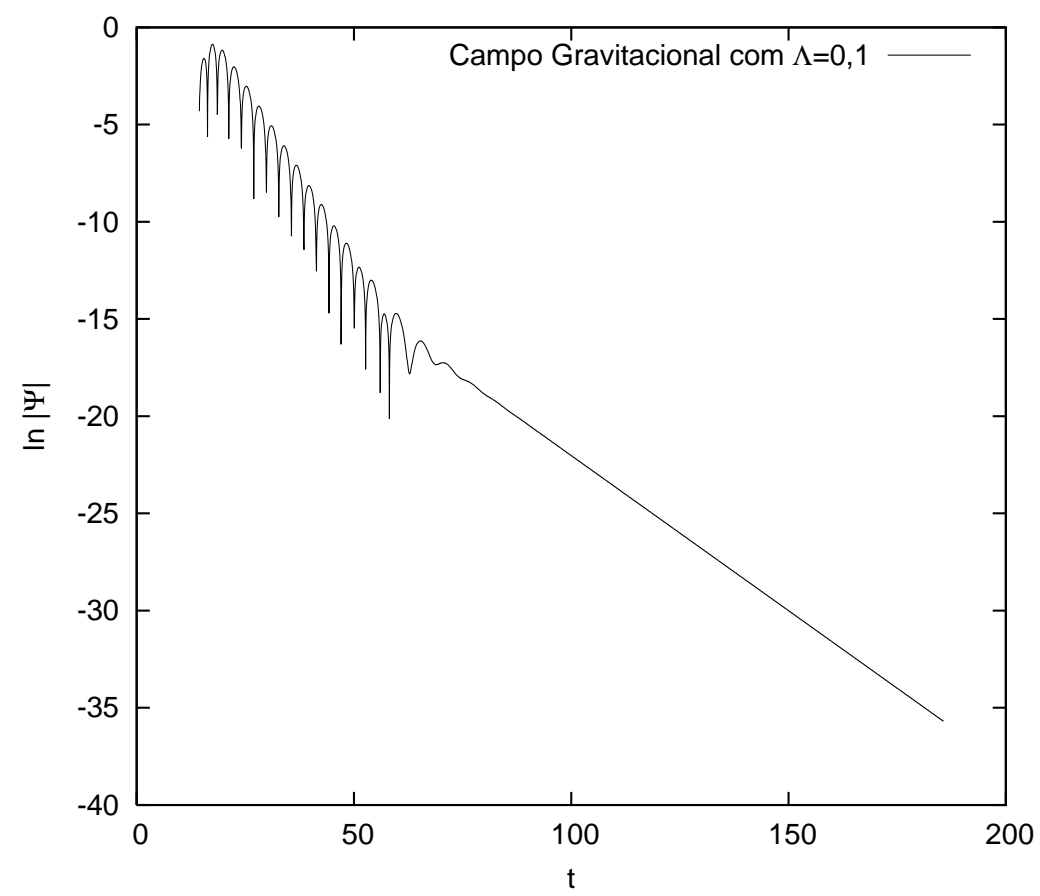

Figura 4.4: Campo gravitacional tensorial $\operatorname{com} Q=0.1$ e $l=2$.

Para este ponto, na tabela acima temos $Q_{C} \sim 0.983$, o que endossa ainda o comportamento dos modos nas imediações de $Q \sim 0.9$ : os modos quasinormais deixam de ser descrescentes com o aumento da carga. Há uma 
suspeita da existência de modos instáveis para valores de $Q$ próximos aos valores extremos $[6,7]$.

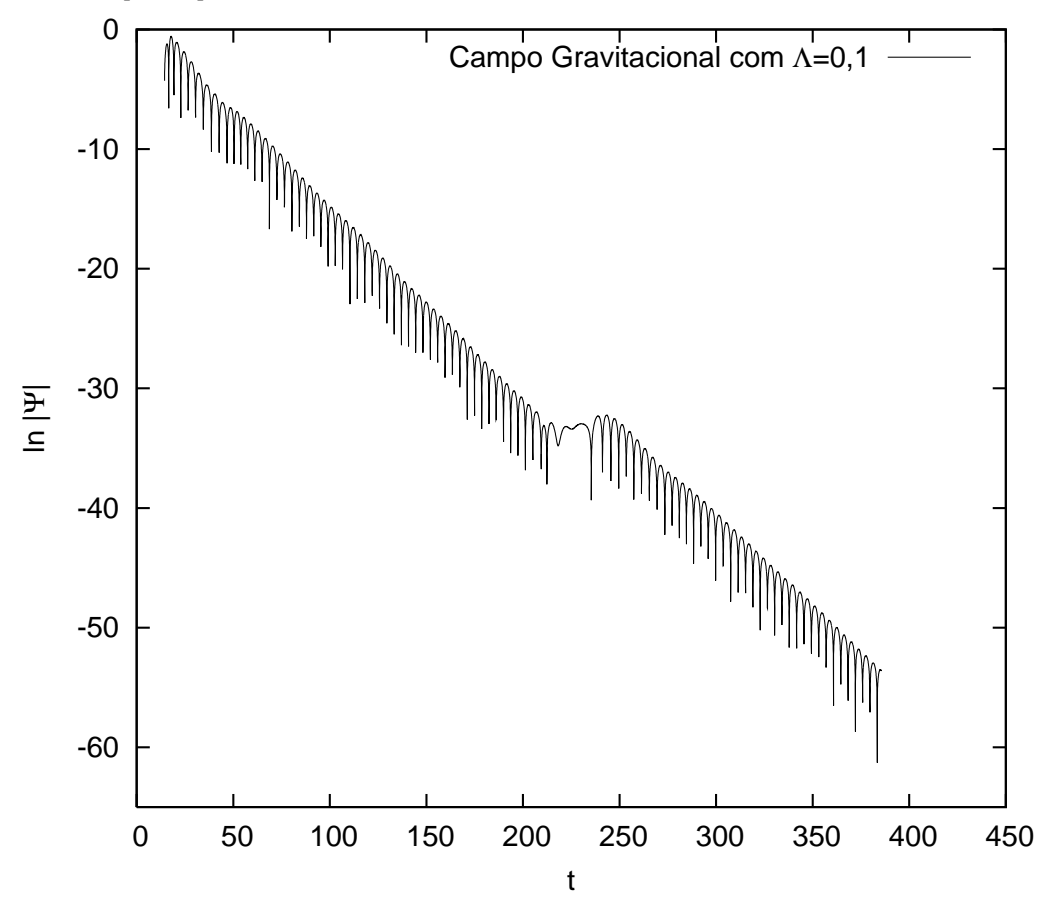

Figura 4.5: Campo gravitacional tensorial $\operatorname{com} Q=0.98$ e $l=2$.

Um perfil do campo típico de perturbação gravitacional tensorial pode ser visto na figura 4.4. Neste perfil, podemos observar o boost inicial, que representa a influência do pacote gaussiano (condições iniciais). Após este boost, temos a fase de oscilação quasi-normal, que no caso do perfil mostrado na figura dura de $t=20$ até $t=60$. Esta fase tende a se tornar cada vez maior, quanto maior a carga do buraco negro, e quanto mais próximo ele está de um buraco negro extremo com horizonte cosmológico no mesmo ponto que o horizonte de eventos.

Finalmente, o terceiro trecho do perfil de oscilação do campo gravitacional é a cauda, que está essencialmente ligada com a presença de um potencial centrífugo, $l(l+D-3)$. Tal fase representa o último aspecto da oscilação gravitacional, e como mostrada no perfil 4.4 denota que o modo de vibração de tal buraco negro é estável à perturbação linear em primeira ordem. No geral, para o cômputo dos modos quasi-normais, a suposição é de que tanto esta 
cauda quanto o período de vibração sejam "bem-comportados" em sentido de que ambos devem decair com o tempo.

Embora da estabilidade, a cauda na figura 4.4 segue uma reta, diferentemente da lei de potência, válida para os casos assintóticamente planos. O mesmo comportamento pode ser encontrado em [69, 65].

A presença de modos instáveis, cuja cauda ou a fase quasi-normal cresçam com a evolução temporal denota que a análise da perturbação em primeira ordem é inconclusiva, para se atestar o caráter final da geometria do espaçotempo embora seja uma forte indicação da instabilidade da solução [8].

A existência de modos instáveis em uma dada geometria está associada ainda, há existência de intervalos em que o potencial gravitacional assume valores negativos: é condição necessária (mas não suficiente), para que haja modos que cresçam com o tempo a existência de um intervalo $[a, b] \epsilon r$, de maneira que $V(r)<0$. Este é o caso da geometria de Reissner-Nordström-de Sitter em 5 dimensões.

Em buracos negros cujos horizontes internos coalesçam, a fase de decaimento tende a se prolongar ao infinito. Por exemplo, no perfil 4.5, podemos notar a tendência de que a fase quasi-normal se prolongue por longos períodos de tempos, quanto mais próximo o buraco negro está de um buraco negro extremo. Em todos os perfis calculados para valores de carga menores do que a carga de um buraco negro extremo (com horizontes de evento e de Cauchy no mesmo ponto), a característica peculiar é a de que a fase quasi-normal é tanto maior quanto mais próximo da carga $Q_{C}$. Para este perfil temos que $\omega=0.895+0.132 i$, sendo sua carga $99,7 \%$ do valor $Q_{C}$.

Quando aumentamos o valor da constante cosmológica, a tendência é de que tanto a parte real quanto a imaginária dos modos quasi-normais diminuam. Por exemplo, para $\Lambda=0.05$ e $\Lambda=0.167$, listamos os modos nas tabelas 4.2 e 4.3. Para o primeiro caso, o valor máximo da carga, para o qual o horizonte de eventos e cosmológico coalescem é $Q \sim 2.124$, e para o segundo $Q=\frac{5}{2 \sqrt{6}} \sim 1.021$.

O comportamento geral dos modos quasi-normais para estes dois valores de $\Lambda$ é similar ao demonstrado para a tabela 4.1, uma vez que, com a aproximação de $Q$ de seu valor máximo, a frequência natural de vibração tende 
Tabela 4.2: Modos quasi-normais para um buraco negro com $\Lambda=0.05 \mathrm{e}$ $l=2$.

\begin{tabular}{|c|c|c|c|c|c|}
\hline$Q$ & $\omega_{R}$ & $\omega_{I}$ & $Q$ & $\omega_{R}$ & $\omega_{I}$ \\
\hline 0.1 & 1.013 & 0.346 & 0.2 & 1.000 & 0.334 \\
\hline 0.3 & 0.985 & 0.292 & 0.4 & 0.973 & 0.309 \\
\hline 0.5 & 0.934 & 0.287 & 0.6 & 0.898 & 0.265 \\
\hline 0.7 & 0.853 & 0.243 & 0.8 & 0.794 & 0.257 \\
\hline 0.9 & 0.723 & 0.146 & 1.0 & 0.732 & 0.147 \\
\hline 1.1 & 0.678 & 0.152 & 1.2 & 0.575 & 0.165 \\
\hline 1.3 & 0.524 & 0.153 & 1.4 & 0.470 & 0.143 \\
\hline 1.5 & 0.415 & 0.131 & 1.6 & 0.362 & 0.116 \\
\hline 1.7 & 0.312 & 0.102 & 1.8 & 0.259 & 0.0892 \\
\hline 1.9 & 0.202 & 0.0693 & 2.0 & 0.147 & 0.0473 \\
\hline
\end{tabular}

Tabela 4.3: Modos quasi-normais para um buraco negro com $\Lambda=0.167 \mathrm{e}$ $l=2$.

\begin{tabular}{|c|c|c|c|c|c|}
\hline$Q$ & $\omega_{R}$ & $\omega_{I}$ & $Q$ & $\omega_{R}$ & $\omega_{I}$ \\
\hline 0.1 & 0.749 & 0.278 & 0.15 & 0.743 & 0.274 \\
\hline 0.2 & 0.736 & 0.269 & 0.25 & 0.726 & 0.262 \\
\hline 0.3 & 0.714 & 0.253 & 0.35 & 0.700 & 0.243 \\
\hline 0.4 & 0.683 & 0.232 & 0.45 & 0.663 & 0.219 \\
\hline 0.5 & 0.640 & 0.205 & 0.55 & 0.613 & 0.191 \\
\hline 0.6 & 0.583 & 0.175 & 0.65 & 0.549 & 0.155 \\
\hline 0.7 & 0.522 & 0.137 & 0.75 & 0.477 & 0.103 \\
\hline 0.8 & 0.411 & 0.0879 & 0.83 & 0.380 & 0.103 \\
\hline 0.86 & 0.353 & 0.0771 & 0.89 & 0.326 & 0.0706 \\
\hline 0.92 & 0.291 & 0.0675 & 0.95 & 0.226 & 0.0501 \\
\hline 0.98 & 0.168 & 0.0441 & 1.01 & 0.0997 & 0.00256 \\
\hline
\end{tabular}


a zero.

Também, nas proximidades do buraco negro extremo com dois horizontes de eventos no mesmo ponto, este comportamento não é reproduzido, e a frequência varia para qualquer valor de $\Lambda$. Os valores de $Q_{C}$ quando $\Lambda=0.05$ e $\Lambda=0.167$ são $Q_{C} \sim 0.949$ e $Q_{C} \sim 0.816$. A característica peculiar em relação a este tipo de buraco negro extremo é a de que, ainda que $\Lambda$ varie bruscamente, $Q_{C}$ variará de maneira mais suave, ou seja, a influência da constante cosmológica é mais perceptível para buracos negros extremos em que os dois horizontes a coalescerem são o cosmológico e o de eventos.

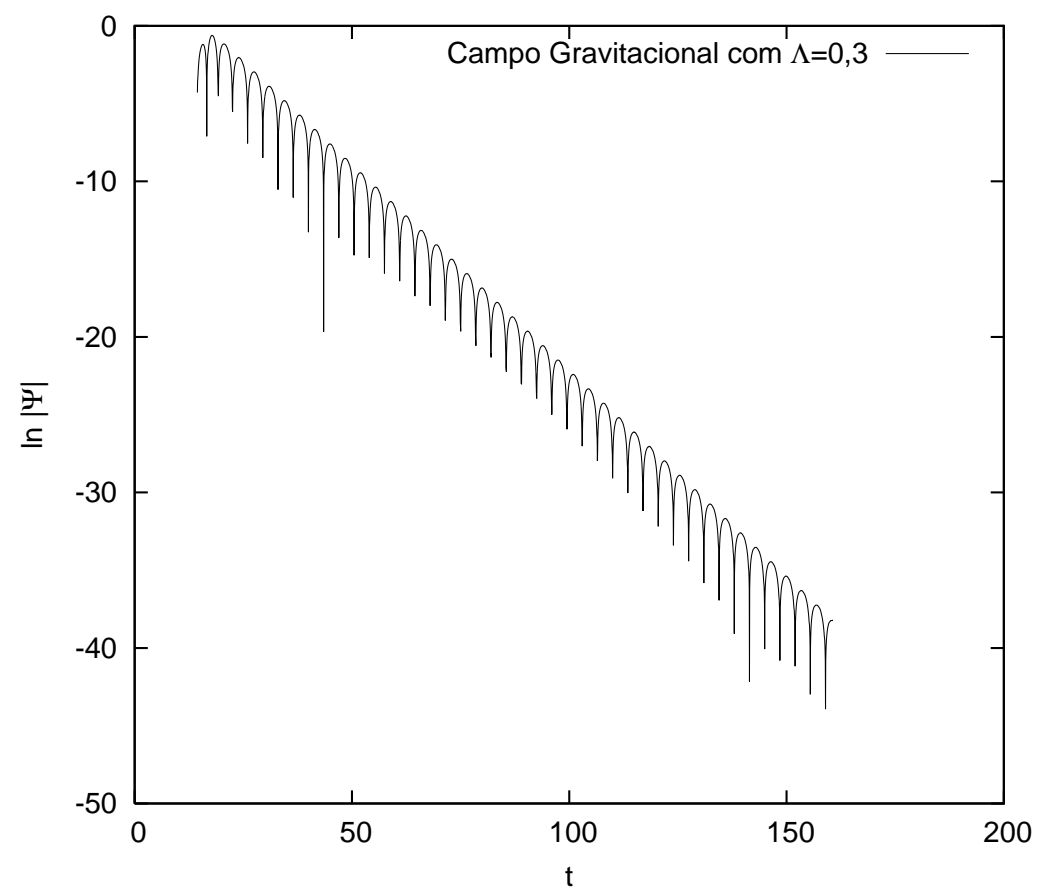

Figura 4.6: Campo gravitacional tensorial $\operatorname{com} Q=0.6$ e $l=2$.

O perfil típico do campo, tanto para $\Lambda=0.05$, quanto para $\Lambda=0.167$ é similar ao perfil 4.4. Para $\Lambda=0.05$, a figura 4.7 representa o campo gravitacional com $Q=0.6$. A diferença acontece contudo na oscilação quasinormal, que tende a se estender por um tempo muito maior, quanto maior o valor de $\Lambda$. O fato de a oscilação perdurar por longos períodos de tempo torna complicado a obtenção da cauda do perfil em questão. Como a precisão máxima a que um programa em fortran pode chegar é dar ordem de $10^{-14}$, 
além do erro numérico natural feito pelo cálculo da coordenada tartaruga, é impossível ir além de certo ponto na integração numérica (sendo, portanto extremamente complicado a obtenção das caudas para certos perfis).

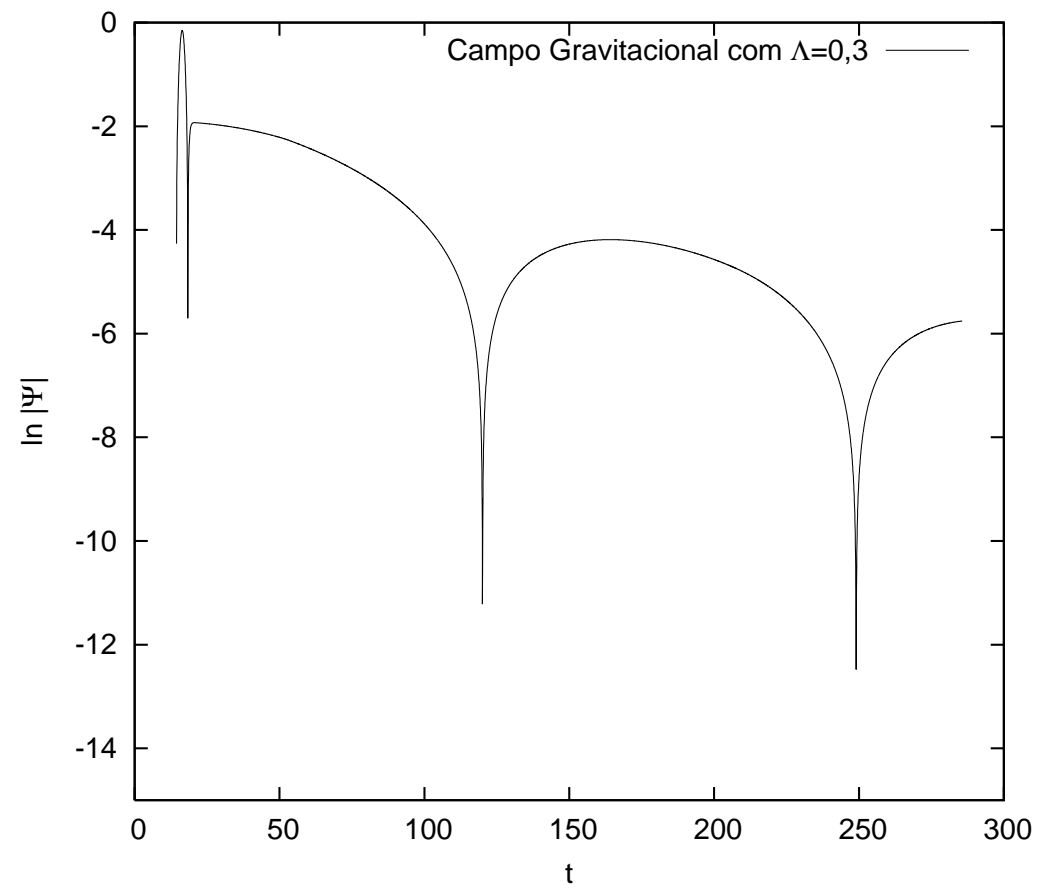

Figura 4.7: Campo gravitacional tensorial $\operatorname{com} Q=0.6$ e $l=2$.

Em 4.7 é perceptível o comportamento do campo quando $Q \sim Q_{\max }$ : para este perfil, $Q=2.12$, o que corresponde a 99,8\% do valor máximo da carga, quando os horizontes de eventos e cosmológico estão muito próximos um do outro: $r_{h} \sim 2.98$ e $r_{C} \sim 3.18$.

A última propriedade analisada com o potencial tensorial foi a influência do momento angular da perturbação, $l$. Na tabela 4.4, estão listados as vibrações para três diferentes valores de $l$.

O perfil de campos para diferentes valores de $l$ pode ser encontrado nas figuras 4.8 e 4.9 , e um zoom destes campos em 4.10 e 4.11. A diferença entre as frequências de modos com diferentes valores de momento angular é notória, sendo entretanto o amortecimento bastante parecido no três casos.

Nas próximas seções, estudaremos o caso da perturbação gravitacional vetorial, que representa dois potenciais diferentes. 
Tabela 4.4: Modos quasi-normais para um buraco negro com $\Lambda=0.08 \mathrm{e}$ diferentes valores de $l$.

\begin{tabular}{|c|c|c|c|}
\hline$Q$ & $\omega(l=2)$ & $\omega(l=3)$ & $\omega(l=4)$ \\
\hline 0.1 & $0.938+0.328 \mathrm{i}$ & $1.480+0.314 \mathrm{i}$ & $1.970+0.310 \mathrm{i}$ \\
\hline 0.2 & $0.928+0.319 \mathrm{i}$ & $1.464+0.307 \mathrm{i}$ & $1.949+0.303 \mathrm{i}$ \\
\hline 0.3 & $0.910+0.306 \mathrm{i}$ & $1.437+0.294 \mathrm{i}$ & $1.913+0.291 \mathrm{i}$ \\
\hline 0.4 & $0.885+0.289 \mathrm{i}$ & $1.398+0.278 \mathrm{i}$ & $1.862+0.274 \mathrm{i}$ \\
\hline 0.5 & $0.851+0.267 \mathrm{i}$ & $1.347+0.257 \mathrm{i}$ & $1.795+0.254 \mathrm{i}$ \\
\hline 0.6 & $0.809+0.244 \mathrm{i}$ & $1.284+0.235 \mathrm{i}$ & $1.712+0.232 \mathrm{i}$ \\
\hline 0.7 & $0.757+0.219 \mathrm{i}$ & $1.209+0.212 \mathrm{i}$ & $1.613+0.209 \mathrm{i}$ \\
\hline
\end{tabular}
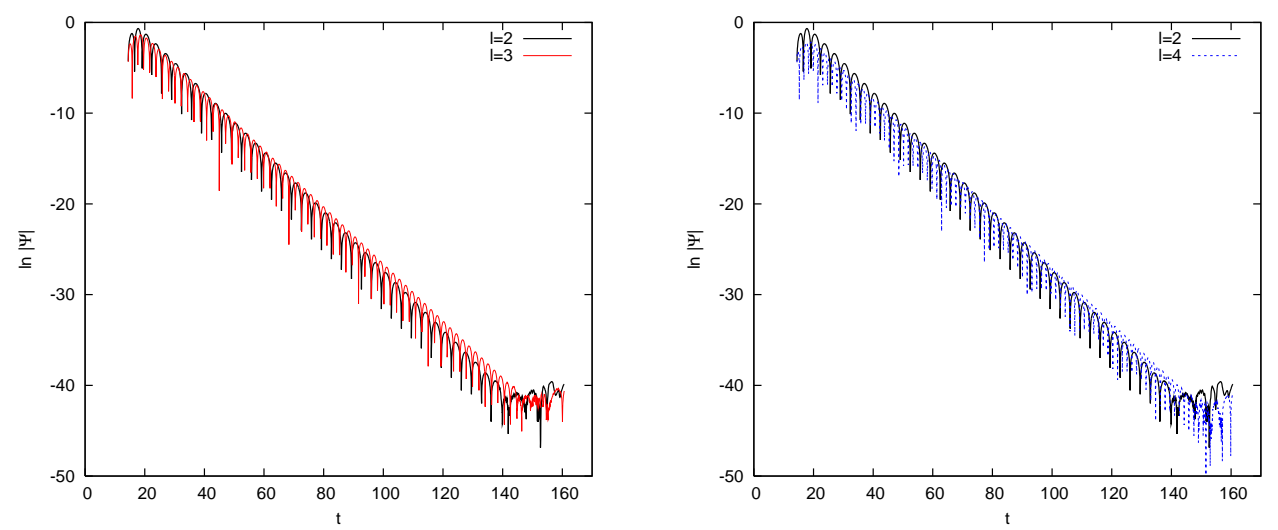

Figura 4.8: Campo gravitacional Figura 4.9: Campo gravitacional com $\Lambda \sim 0.083$ e $Q=0.5$, para dois com $\Lambda \sim 0.083$ e $Q=0.5$, para dois valores de momento angular, $l=2 \mathrm{e}$ valores de momento angular, $l=2 \mathrm{e}$ $l=3$. $l=4$.

\subsection{Perturbação Vetorial}

De maneira similar à que fizemos para a pertubação tensorial, no caso vetorial podemos compor a perturbação da métrica e do tensor energia momento em grupos de componentes de acordo com a simetria da variedade. Temos

$$
\begin{aligned}
& \delta g_{a b}=0, \quad \delta g_{a i}=r f_{a} V_{i}, \quad \delta g_{i j}=2 r^{2} H_{T} V_{i j} \\
& \delta T_{a b}=0, \quad \delta T_{a i}=r \tau^{a} V_{i}, \quad \delta T_{i j}=\tau V_{i j} .
\end{aligned}
$$



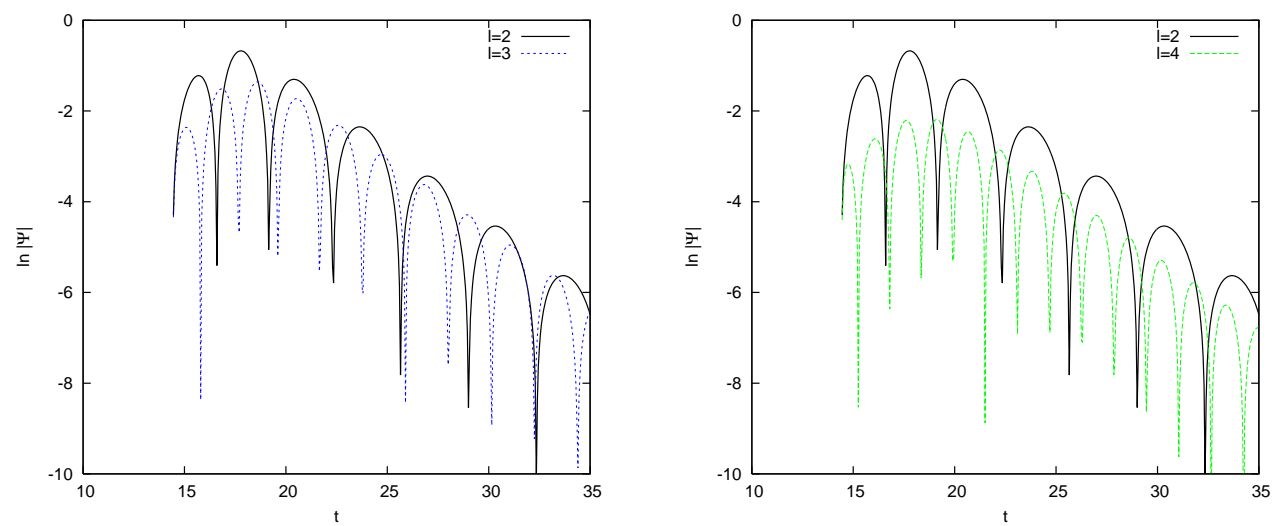

Figura 4.10: "Zoom" de campo gra- Figura 4.11: "Zoom" de campo gravitacional com $\Lambda \sim 0.083$ e $Q=0.5$, vitacional com $\Lambda \sim 0.083$ e $Q=0.5$, para dois valores de momento angu- para dois valores de momento angular, $l=2$ e $l=3$. lar, $l=2$ e $l=4$.

do que, com uma redefinição de variável dada por $F_{a}=f_{a}+r D_{a}\left(H_{T} / k_{V}\right)$ emergem $a+2$ equações de campo representadas por

$$
\begin{aligned}
D_{a}\left[r^{D-1} \varepsilon^{c b} r D_{a}\left(F_{a} / r\right)\right] & =m_{V} r^{D-3} \varepsilon_{a b} F^{b}-2 r^{D-1} \varepsilon_{a b} \tau^{b} \\
k_{V} D_{a}\left(r^{D-3} F^{a}\right) & =-r^{D-2} \tau \\
D_{a}\left(r^{D-1} \tau^{a}\right) & =-\frac{m_{V}}{2 k_{V}} r^{D-2} \tau
\end{aligned}
$$

com a constante $m_{v}=k_{V}^{2}-(D-3)$. $k_{v}^{2}$ é o autovalor da equação de Lichnerowicz, a menos de uma constante: $\left(\Delta_{L}-k_{V}^{2}-(D-3)\right) V_{i j}=0$. Com uma nova redefinição da variável $F$, dada por $r^{D-3} F^{a}=\varepsilon^{a b} D_{b} \Omega+\frac{2}{m_{V}} r^{D-2} \varepsilon^{a b} D_{a}\left(r \tau_{b}\right)$, obtemos uma equação no formato

$$
r^{D-2} D_{a}\left[\frac{D^{a} \Omega}{r^{D-2}}\right]-\frac{m_{V}}{r^{2}} \Omega=-\frac{2}{m_{V}} r^{D-2} \varepsilon^{a b} D_{a}\left(r \tau_{b}\right)
$$

com a condição extra de que $m_{V} \neq 0$. Os modos de perturbação gerados por $m_{V}=0$ representam configurações de gauge puro, para as quais não há liberdade dinâmica [63].

Para obter uma equação de variável única, o próximo passo é considerarmos rotações de gauge devido ao tensor momento-energia do campo ele- 
tromagnético. Isto pode ser feito modificando-se a componente $\tau_{b}$, acrescentando um termo relativo a um potencial $A$, com uma prescrição semelhante à de Regge-Wheeler [4]: $\tau_{a} \rightarrow \tau_{a}-\frac{E_{0}}{r} \varepsilon_{a b} D^{b} A$. Neste caso, com uma substituição semelhante para o campo, $\Omega \rightarrow \Omega-\frac{2 E_{0}}{m_{V}} A r^{D-2}$, obtemos a equação de perturbação

$$
r^{D-2} D_{a}\left(\frac{1}{r^{D-2}} D^{a} \Omega\right)-\frac{m_{V}}{r^{2}} \Omega=\frac{E_{0} r^{D-2}}{r^{2}} A-\frac{2}{m_{V}} r^{D-2} \varepsilon^{a b} D_{a}\left(r \tau_{b}\right) .
$$

Finalmente, com uma última redefinição dos campos $\Omega$ e $A$,

$$
\Psi=\frac{Q m_{V}}{\left(D^{2}-4 D+3\right) M+\delta} r^{(D-2) / 2} \Omega+\frac{Q}{E_{0} r^{D / 2}} A,
$$

$\operatorname{com} \delta^{2}=\left(D^{2}-4 D+3\right)^{2} M^{2}+2(D-2)(D-3) m_{V} Q^{2}$, conseguimos a equação de campo para uma variável única com potencial, similar à da seção 4.2,

$$
\square \Psi+\frac{V}{g_{t t}} \Psi=0
$$

sendo $V$ o potencial dado por

$$
\begin{aligned}
V= & -\frac{g_{t t}}{r^{2}}\left[k_{V}^{2}+\frac{D^{2}-6 D+8}{4}-\frac{(D-2)(D-4)}{4} \Lambda r^{2}\right. \\
& \left.+\frac{(D-2)(5 D-12)}{4 r^{2 D-6}}+\frac{\left(-D^{2} / 2+2 D-3\right) M+\delta}{r^{D-3}}\right]
\end{aligned}
$$

Na próxima seção, analisaremos os resultados dos modos quasi-normais para este potencial.

\subsection{Resultados com a Perturbação Vetorial}

Para a análise dos modos quasi-normais com um potencial vetorial, usamos o mesmo método de integração em coordenadas nulas descrito no início do capítulo: transformamos a equação de onda em (4.44) com o uso de coordenadas nulas para uma outra equação com o mesmo formato de (4.15). Neste caso, podemos usar as mesmas condições de contorno (4.19), para a obtenção 
Tabela 4.5: Modos quasi-normais de uma perturbação gravitacional vetorial para o buraco negro de Reissner-Nordström de Sitter, com $\Lambda=0.0167 \mathrm{e}$ $l=2$.

\begin{tabular}{|c|c|c|c|c|c|}
\hline$Q$ & $\omega_{R}$ & $\omega_{I}$ & $Q$ & $\omega_{R}$ & $\omega_{I}$ \\
\hline 0.2 & 1.354 & 0.337 & 0.4 & 1.368 & 0.314 \\
\hline 0.6 & 1.360 & 0.276 & 0.8 & 1.301 & 0.230 \\
\hline 0.9 & 1.300 & 0.190 & 0.983 & 1.226 & 0.158 \\
\hline 1.0 & 1.189 & 0.162 & 1.2 & 1.038 & 0.192 \\
\hline 1.5 & 0.837 & 0.164 & 2.0 & 0.587 & 0.129 \\
\hline 2.5 & 0.415 & 0.0966 & 3.0 & 0.282 & 0.0681 \\
\hline 3.5 & 0.155 & 0.0340 & 3.8 & 0.0173 & 0.0110 \\
\hline
\end{tabular}

dos perfis de campo e dos modos quasi-normais em toda a escala de valores possíveis de $\Lambda$ e $Q$.

Os resultados da perturbação vetorial para o buraco negro de ReissnerNordström de Sitter com um valor pequeno para a cosmolgógica $(\Lambda=1 / 60)$, estão na tabela 4.5. Diferentemente da perturbação tensorial, quando da presença de um potencial gravitacional oriundo de uma perturbação vetorial, a frequência de vibração da resposta de uma perturbação gravitacional é menos sensível à buracos negros com diferentes valores de carga, e oscila ligeiramente em torno do ponto $\omega_{R} \sim 1.3$, para buracos negros com carga menor do que $Q_{C}$, quando da coalisão dos dois horizontes de eventos.

A característica semelhante, contudo, é o decréscimo da atenuação dos modos, com o aumento da carga, de maneira que quando a carga tende a seu valor máximo (caso em que o horizonte de eventos e cosmológico coalescem), a atenuação tende a zero. Também, após a "região" de buraco negro extremo com os dois horizontes no mesmo ponto (ou seja, em todo o escopo $Q<Q_{C}$ e $Q \geq Q_{C}$ ), a parte real de $\omega$ começa a diminuir mais rapidamente do que para o potencial tensorial, tendendo a zero, conforme $Q$ vai para seu valor máximo, $Q_{S}$.

O perfil do campo gravitacional vetorial, com $\Lambda=1 / 60$ é mostrado na figura 4.12. No gráfico, é perceptível a diminuição da atenuação e da frequência 


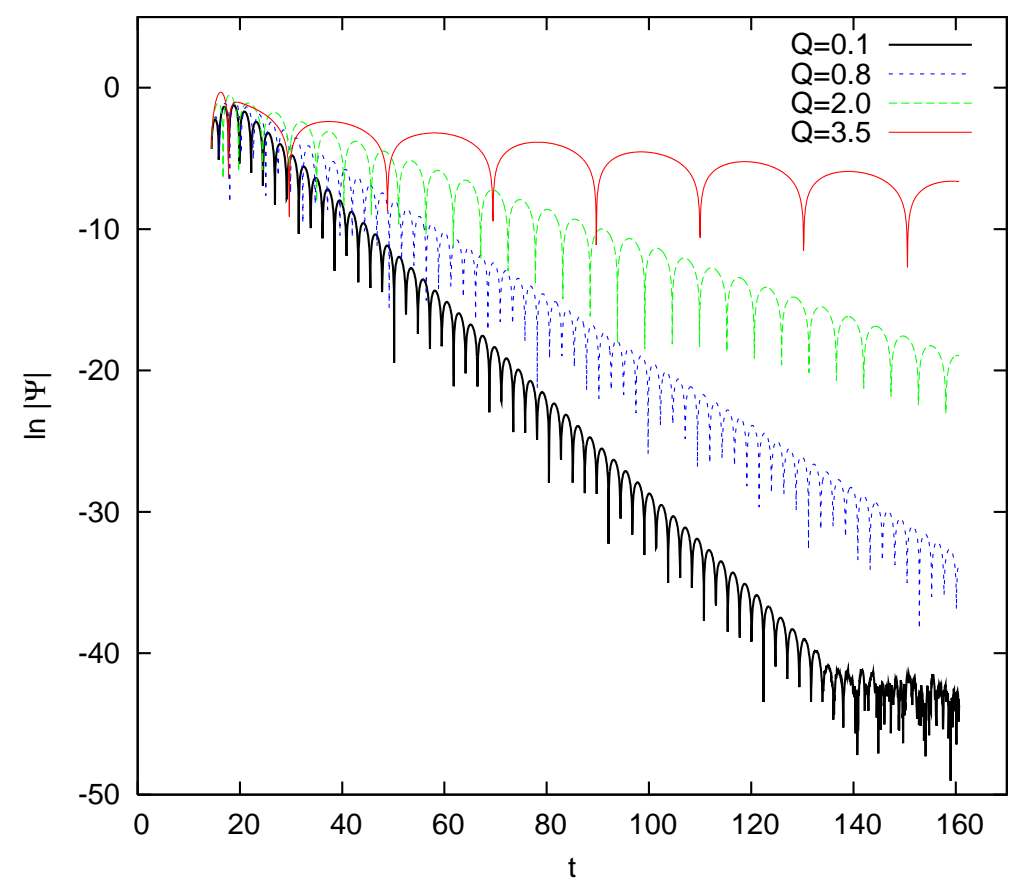

Figura 4.12: Campo gravitacional vetorial para valores diferentes de $Q, \Lambda=$ 0.0167 e $l=2$.

da onda quando do aumento da carga para valores maiores do que $Q_{C}$. Outra característica peculiar é a proximidade entre as frequências da perturbação entre valores de carga diferentes, para os quais $Q<Q_{C}$, como se pode ver nos dois primeiros perfis, para $Q=0.1$ e $Q=0.8$.

Na tabela 4.6 estão listados os modos quasi-normais com $\Lambda=0.167$, para os quais $Q_{C} \sim 0.817$. Novamente, a parte real dos modos oscila ligeiramente, quando $Q<<Q_{C}$, e quando se aproxima deste valor tende a decrescer. Diferentemente do caso com $\Lambda=0.0167$, contudo, esta função continua a oscilar ligeiramente, após $Q \sim Q_{C}$, decaindo mais lentamente, conforme $Q$ se aproxima de seu valor máximo. A parte imaginária apresenta o mesmo comportamento observado para as outras perturbação tanto tensoriais quanto vetoriais.

Na figura 4.13, podemos ver diferentes perfis de campo, em função da carga. As características destes perfis são similares àquelas da figura 4.12, exceto pelo fato de a extensão de valores válidos para a carga ser muito 
Tabela 4.6: Modos quasi-normais de uma perturbação gravitacional vetorial para o buraco negro de Reissner-Nordström de Sitter, com $\Lambda=0.0167 \mathrm{e}$ $l=2$.

\begin{tabular}{|c|c|c|c|c|c|}
\hline$Q$ & $\omega_{R}$ & $\omega_{I}$ & $Q$ & $\omega_{R}$ & $\omega_{I}$ \\
\hline 0.1 & 1.004 & 0.257 & 0.15 & 1.005 & 0.254 \\
\hline 0.2 & 1.005 & 0.249 & 0.25 & 1.004 & 0.243 \\
\hline 0.3 & 1.002 & 0.236 & 0.35 & 0.997 & 0.227 \\
\hline 0.4 & 0.990 & 0.217 & 0.45 & 0.979 & 0.206 \\
\hline 0.5 & 0.963 & 0.193 & 0.55 & 0.941 & 0.178 \\
\hline 0.6 & 0.913 & 0.163 & 0.65 & 0.878 & 0.147 \\
\hline 0.7 & 0.831 & 0.136 & 0.75 & 0.772 & 0.104 \\
\hline 0.8 & 0.739 & 0.102 & 0.83 & 0.667 & 0.0709 \\
\hline 0.86 & 0.630 & 0.0735 & 0.89 & 0.452 & 0.0512 \\
\hline 0.92 & 0.475 & 0.0530 & 0.95 & 0.409 & 0.0578 \\
\hline 0.98 & 0.282 & 0.0357 & 1.01 & 0.160 & 0.02247 \\
\hline
\end{tabular}

menor, o que torna o intervalo de entre os dois buracos negros extremos muito menor, e portanto resultando ambas as situação extremas em modos de vibração com atenuação semelhante.

$\mathrm{Na}$ tabela 4.7, vemos os modos quasi-normais para diferentes valores de momento angular $l$, e o comportamento de que a parte imaginária pouco varia para diferentes valores de $l$ é similar ao encontrado na perturbação tensorial. A parte real aumenta ligeiramente com o aumento da carga, para valores pequenos de carga, e a partir de um valor crítico de carga, o qual depende do valor de $l$, começa a decrescer novamente. A parte imaginária por sua vez pouco varia para valores pequenos da carga.

Quando $\Lambda=0.083$, o valor para o qual o buraco negro se torna extremo é $Q_{C}=0.93$, o que indica a ausência de modos instáveis mesmo em regiões próximas à de buracos negros extremos.

As figuras 4.14 e 4.15 representam a perturbação do campo gravitacional para diferentes valores de $l$. É possível com elas visualizar a mesma atenuação para os modos, ainda que $l$ varie, contudo uma frequência diferente, dependente de $l$.

Em todos os modos analisados para 5 dimensões, não foram encontra- 


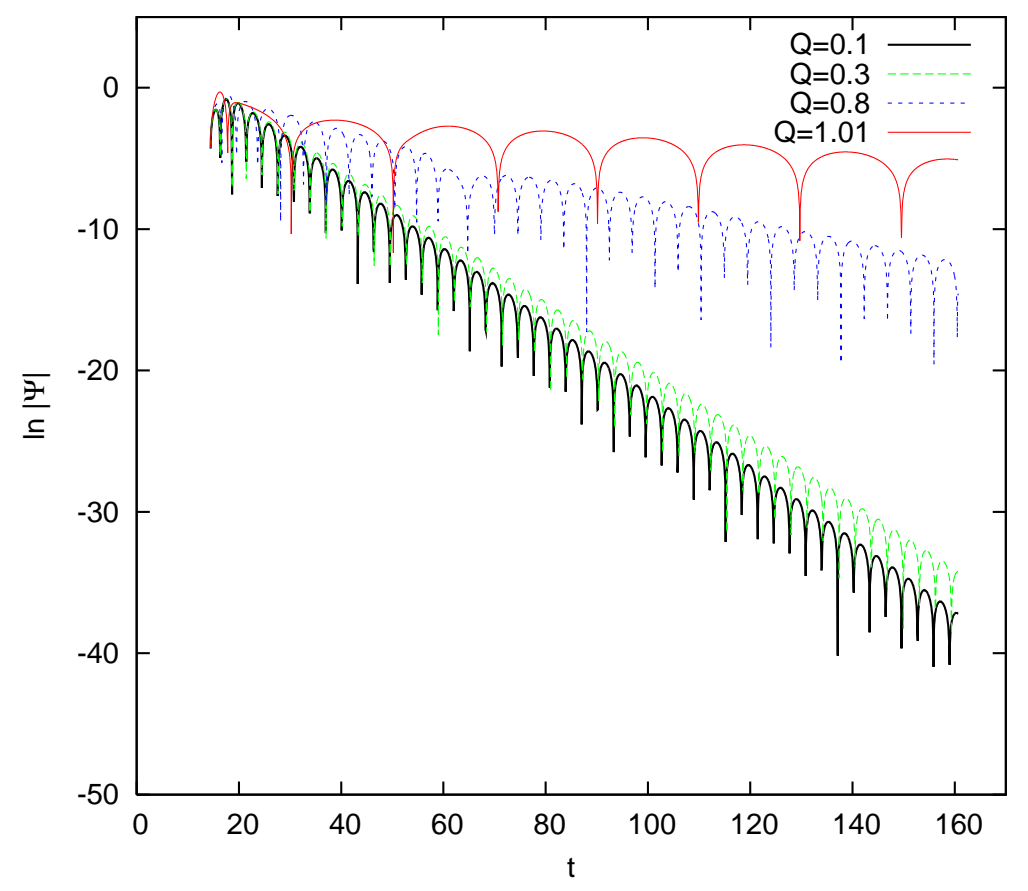

Figura 4.13: Campo gravitacional vetorial para valores diferentes de $Q, \Lambda=$ 0.167 e $l=2$.

dos vestígios de modos instáveis, como por exemplo em [6, 7], encontramos modos instáveis para o potencial gravitacional escalar com $D \geq 7$. O único suporte para a existência de modos instáveis está em alguns modos para os quais, depois de um longo período de integração, aparentemente o modo volta a crescer ao invés de decair (por exemplo 4.9 e 4.10). Tal comportamento contudo, corresponde ao limite numérico do programa utilizado uma vez que a precisão não ultrapassa a décima quinta casa para todos os cálculos de integração (e. g. coordenada tartaruga, valor do potencial, valor do campo, etc), e neste sentido não é mais do que "ruído". Tal limitação é momentaneamente inerente a estrutura do programa utilizado para o cálculo (fortran, versão gnu), mesmo que com a maior precisão possível para todas as variáveis (precisão estendida). As caudas obtidas quando a precisão necessaria para sua obtenção não ultrapassa $10^{-15}$, seguem uma exponencial decrescente, como em [69]. 
Tabela 4.7: Modos quasi-normais de uma perturbação gravitacional vetorial para o buraco negro de Reissner-Nordström de Sitter, com $\Lambda=0.0833$ e diferentes valores de $l$.

\begin{tabular}{|c|c|c|c|}
\hline$Q$ & $\omega(l=2)$ & $\omega(l=3)$ & $\omega(l=4)$ \\
\hline 0.1 & $1.238+0.316 \mathrm{i}$ & $1.716+0.315 \mathrm{i}$ & $2.181+0.315 \mathrm{i}$ \\
\hline 0.2 & $1.243+0.310 \mathrm{i}$ & $1.720+0.309 \mathrm{i}$ & $2.183+0.309 \mathrm{i}$ \\
\hline 0.3 & $1.248+0.299 \mathrm{i}$ & $1.720+0.299 \mathrm{i}$ & $2.179+0.298 \mathrm{i}$ \\
\hline 0.4 & $1.250+0.284 \mathrm{i}$ & $1.712+0.284 \mathrm{i}$ & $2.161+0.283 \mathrm{i}$ \\
\hline 0.5 & $1.243+0.265 \mathrm{i}$ & $1.619+0.264 \mathrm{i}$ & $2.125+0.264 \mathrm{i}$ \\
\hline 0.6 & $1.225+0.241 \mathrm{i}$ & $1.653+0.241 \mathrm{i}$ & $2.069+0.242 \mathrm{i}$ \\
\hline 0.7 & $1.191+0.216 \mathrm{i}$ & $1.595+0.217 \mathrm{i}$ & $1.989+0.218 \mathrm{i}$ \\
\hline 0.8 & $1.131+0.189 \mathrm{i}$ & $1.512+0.192 \mathrm{i}$ & $1.884+0.192 \mathrm{i}$ \\
\hline 0.9 & $1.199+0.136 \mathrm{i}$ & $1.456+0.154 \mathrm{i}$ & $1.769+0.113 \mathrm{i}$ \\
\hline
\end{tabular}
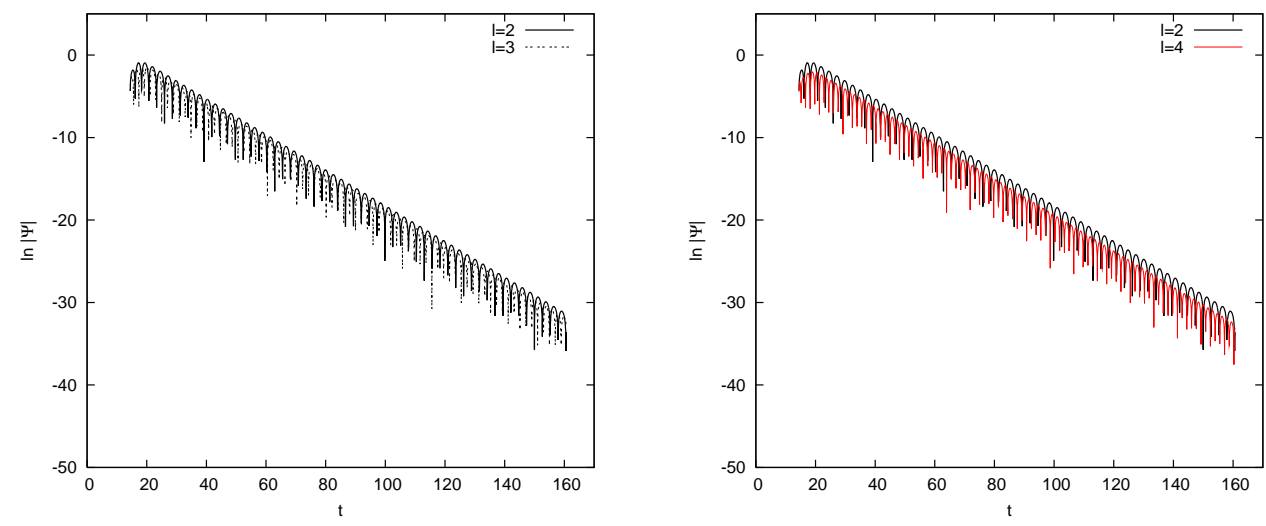

Figura 4.14: "Zoom" de campo gra- Figura 4.15: "Zoom" de campo gravitacional com $\Lambda \sim 0.083$ e $Q=0.5$, vitacional com $\Lambda \sim 0.083$ e $Q=0.5$, para dois valores de momento angu- para dois valores de momento angular, $l=2$ e $l=3$. lar, $l=2$ e $l=4$. 


\section{Capítulo 5}

\section{Apontamentos Finais}

Ao longo deste trabalho, debruçamo-nos sobre o estudo das frequências quasinormais de buracos negros com campos eletromagnéticos.

A determinação dos modos quasi-normais de buracos negros tem uma série de interesses físicos: como ferramente teórica de comparação de futuras medidas de ondas gravitacionais em grandes laboratórios (LISA, VIRGO, etc); como ferramente teórica para a eventual comparação com a área de termodinâmica de buracos negros, e determinação de se os modos de vibração carregam as propriedades termodinâmicas de solução, e, de que maneira isso acontece; como teste de estabilidade do espaço-tempo, podendo denotar a presença de modos que cresçam no domínio temporal, o que pode ser interpretado como um forte indício da instabilidade do espaço-tempo (um teste robusto de uma instabilidade, contudo, pode ser arquitetado apenas com a teoria não-linear, e não com expansões em primeira ordem. Neste sentido, os modos quasi-normais estão limitados a dar uma resposta segura apenas quando as vibrações são estáveis, i. e., decaem com o tempo).

Ocupamo-nos no decorrer dos três capítulos anteriores com cada um destes aspectos, utilizando soluções diferentes, de acordo com o estado da arte em cada subtópico.

Utilizando o buraco negro de Melvin-Ernst (que possui dois parâmetros em sua geometria, a massa e o campo magnético), calculamos os modos de vibração correspondentes à propagação de um campo escalar em tal geometria, 
considerando o campo magnético pequeno. Tal aproximação é válida, visto que buracos negros (astrofísicos) possuem, via de regra, discos de acresção de matéria que geram uma contribuição não nula de campo magnético, que ainda assim é muito menor do que a massa do buraco negro. Demonstramos que a propagação de um campo escalar em tal geometria tem o mesmo potencial da propagação de um campo escalar com massa em uma geometria de Schwarzschild, com a correspondência da massa de tal campo escalar sendo $\mu=2|m| B, m$ o número azimutal de harmônicos esféricos e $B$. Além desta equivalência, a propagação do campo escalar na geometria de Melvin-Ernst tem a propriedade de que o buraco negro se torna um melhor oscilador (melhora o fator de qualidade $Q=\omega_{R} / \omega_{I}$, tanto maior é o campo magnético do espaço-tempo.

Também usando buracos negros com campo magnético de fundo, investigamos a contribuição do campo escalar para a entropia da solução através do método Brick Wall proposto por 't Hooft em 1984. A contribuição do campo magnético é da ordem $B^{2}$, para a divergência ultravioleta, $\epsilon^{-1}$. No caso de buracos negros D-dimensionais, a divergência ultra-violeta tem a mesma forma daquela divergência para Schwarzschild, $\epsilon^{\frac{2-D}{2}}$, tendo a divergência ultravioleta, contudo um formato diferente dado por $\frac{L^{D-3}}{B^{2}}$, de maneira que o campo magnético age diminuindo tal divergência (que para Schwarzschild é dada por $\left.L^{D-1}\right)$.

Com uma solução de Reissner-Nordström em quatro dimensões analisamos as propriedades termodinâmicas do buraco negro e a relação com seus modos de vibração. Utilizando a proposta de Maggiore para a conjectura Hod, de reinterpretação da constante de quantização da área do buraco negro de Schwarzschild, a partir de uma comparação com o oscilador harmônico com atrito (o que dá um sentido semi-clássico e explica algumas críticas da antiga proposta de Hod), calculamos os modos quasi-normais em ReissnerNordström chegando a mostrar que o espaçamento assintótico dos modos se aproxima da mesma quantização que para Schwarzschild.

Além da conjectura Hod modificada, testamos ainda a relação dos modos quasi-normais com a transição de ordem de segunda fase [47], como proposta por Jing et al [49]. No setor de perturbações gravitacionais, a equivalência 
encontrada em [49] demonstrou-se não existir, de maneira que, assim como para a solução de Kerr tal correspondência não é válida, podemos afirmar que se trata de uma coincidência numérica. Não se pode negar que os modos quasi-normais carreguem as propriedades termodinâmicas relacionadas com a transição de ordem de segunda fase, mas se esta característica existe ela é mais profunda do que a proposta em [49].

Finalmente, estudamos no último capítulo, a perturbação gravitacional de um buraco negro de Reissner-Nordström-de Sitter em 5 dimensões, cujos modos quasi-normais, tanto para o potencial tensorial como vetorial, demonstraram-se estáveis, no sentido de que decaem com o tempo. O comportamento particular de cada frequência depende, como esperado dos parâmetros do buraco negro, e em geral, exceto para situações extremas, varia de maneira unívoca com a variação dos parâmetro: por exemplo, com o aumento da carga do buraco negro, tanto $\omega_{R}$ quanto $\omega_{I}$ diminuem, tendendo a zero quando de um buraco negro extremo com horizonte de eventos e cosmológico no mesmo ponto. Além, a influência do momento angular das vibrações é mais sentida em $\omega_{R}$ do que em $\omega_{I}$ : para dados pariametros $M, Q$ e $\Lambda$, a variação de $l$ produz uma grande variação em $\omega_{R}$, mas uma pequena (ainda menor que para o caso de Schwarzschild) variação em $\omega_{I}$.

Como perspectivas futuras, demarcamos algumas idéias passíveis de estudo, em cada um dos trabalhos aqui relatados durante os anos de sua pesquisa:

- A investigação da propagação de campos elétricos ou da perturbação gravitacional em um buraco negro de Schwarzschild imerso em um Universo magnético. A dificuldade em tal caso é dada pela escrita do tensor perturbação gravitacional em respeitando as simetrias do espaço-tempo para o caso da perturbação, e ainda, no desacoplamento das equações de campo para o caso do campo elétrico;

- A verificação da conjectura modificada de Hod para o buraco negro de Kerr com limites diferentes dos calculados em [70], bem como a verificação se a presença de divergências em $d \omega_{R} / d \omega_{I}$ está relacionada com a transição de fase de segunda ordem utilizando outros buracos 
negros como espaços-tempo de teste.

- O cálculo dos modos quasi-normais para um número maior de dimensões do buraco negro de Reissner-Nordström-de Sitter, e a verificação de se a instabilidade encontrada para o potencial escalar acontece também para os potenciais vetorialou tensorial [6, 7]. O cálculo dos modos para o buraco negro de RN-AdS, e a relação com a conjectura AdS-CFT [71, 72, 73]. 


\section{Referências Bibliográficas}

[1] LIGO Scientific Collaboration (online: arXiv:1103.2728), The Advanced LIGO Gravitational Wave Detector.

[2] A. Giazotto, Nucl. Instrum. Meth. A, 289, 518 (1990), The Virgo Project: A Wide Band Antenne For Gravitational Wave Detection.

[3] O. D. Aguiar et al, Class. Quant. Grav., 25, 114042 (2008), The Schenberg gravitational wave detector: The first comissioning runs.

[4] T. Regge, J. A. Wheeler, Phys. Rev., 108, 1063 (1957), Stability of a Schwarzschild Singularity.

[5] K. D. Kokkotas, B. G. Schmidt, Living Rev. Rel., 2, 2 (1999), Quasinormal modes of stars and black holes.

[6] R. A. Konoplya, A. Zhidenko, Phys. Rev. Lett., 103, 161101 (2009), Instability of higher dimensional charged black holes in the de Sitter world.

[7] R. A. Konoplya, A. Zhidenko, Phys. Rev. D, 78, 104017 (2008), Stability of higher dimensional Reissner-Nordström-anti-de Sitter black holes.

[8] R. Gregory, R. Laflamme, Phys. Rev. Lett., 70, 2837 (1993), Black strings and p-branes are unstable.

[9] R. Gregory, Class. Quant. Grav., 17, L125 (2000), Black String Instabilities in Anti-de Sitter space. 
[10] J. M. Bardeen, B. Carter, S. W. Hawking, Commun. Math. Phys., 31, 161 (1973), The four laws of black hole mechanics; S. W. Hawking, Phys. Rev. D, 13, 191 (1976), Black Holes and Thermodynamics

[11] J. D. Bekenstein, I. Lett. Nuovo Cim., 4, 737 (1972), Black Holes and the second law; II. Phys. Rev. D, 7, 2333 (1973), Black holes and Entropy; III. Phys. Rev. D, 9, 3292 (1974), Generalized second law of thermodynamics in black hole physics.

[12] S. W. Hawking, Commun. Math. Phys., 43, 199 (1975), Particle Creation by Black Holes.

[13] F. J. Ernst J. of Math. Phys. (17) No.1, (1976); F. J. Ernst and W. J. Wild J. of Math. Phys. (17) No.2, (1976).

[14] B. K. Harrison J. of Math. Phys. (9) No.11 (1968).

[15] B. Preston and E. Poisson, Phys. Rev. D 74 (2006), A light-cone gauge for black-hole perturbation theory; K. Martel and E. Poisson, Phys. Rev. D 71 (2005), Gravitacional perturbations of the Schwarzschild spacetime: A practical covariant and gauge-invariant formalism; B. Preston E. Poisson Phys. Rev. D 74 (2006), Light-cone coordinates based at a geodesic world line.

[16] F. J. Ernst Phys. Rev. (167) No. 5, (1968), New Formulation of the Axially Symmetric Gravitational Field Problem.

[17] F. J. Ernst Phys. Rev. (168) No. 5, (1968), New Formulation of the Axially Symmetric Gravitational Field Problem II.

[18] F. J. Ernst J. of Math. Phys. (15) No.9, (1974).

[19] M. A. Melvin Phys. Rev. (139) No.1B, (1965).

[20] R. A. Konoplya, Phys.Lett.B 644 (2007), Magnetized black hole as a gravitational lens. 
[21] A. Zhidenko, Quasi-normal modes for black hole solutions unkonown in a analytical form.

[22] M. Agoup, E. Radu and R. Slagter, Mod. Phys. Lett. A 20 (2005).

[23] F. R. Tangherlini, Il Nuovo Cimento Vol.XXVII, N.3, (1963), Schwarzschild Field in $n$ Dimensions and The Dimensionality of Space Problem.

[24] M. Ortaggio, JHEP 048 gr-qc/0410048 (2005).

[25] G. 't Hooft, Nucl. Phys. B, 256 (1985), On the Quantum Structure of a Black Hole.

[26] E. Abdalla, B. Cuadros-Melgar, A. B. Pavan, C. Molina Nucl. Phys. B, 752, 40 (2006), Stability and Thermodynamics of brane black holes.

[27] E. Radu, Mod. Phys. Lett. A, 17 (2002), A Note on Schwarzschild Black Hole Thermodynamics in a Magnetic Universe.

[28] R. Konopolya, R. D. Fontana Phys. Lett. B, 659 (2008), Quasinormal modes of black holes immersed in a strong magnetic field.

[29] L. Susskind, J. Uglum Phys. Rev. D, 50 (1994), Black hole entropy in canonical quantum gravity and superstring theory.

[30] E. Abdalla, L. A. Correa-Borbonet, Mod. Phys. Lett. A 16 (2001), Black hole entropy by the brick wall method in four-dimensions and five-dimensions with $\mathrm{U}(1)$ charge.

[31] M. Agop, E. Radu, R. Slagter, Mod. Phys. Lett. A, 20, 1077 (2005), On Ernst black holes with a dilaton potential.

[32] B. Preston and E. Poisson, Phys.Rev.D 74 (2006), A light-cone gauge for black-hole perturbation theory; K. Martel and E. Poisson, Phys.Rev.D 71 (2005), Gravitacional perturbations of the Schwarzschild spacetime: A practical covariant and gauge-invariant formalism; B. Preston E. Poisson Phys.Rev.D 74 (2006), Light-cone coordinates based at a geodesic world line. 
[33] R. A. Konoplya, Phys.Rev.D 74 (2006), Particle motion around magnetized black holes: Preston-Poisson space-time.

[34] H-P. Nollert, Phys. Rev. D 47, 5253 (1993), Quasinormal modes of Schwarzschild black holes: The determination of quasinormal frequencies with very large imaginary parts.

[35] E. Abdalla, K. H. C. Castello-Branco, A. Lima-Santos, Mod. Phys. Lett. A, 18, 1435 (2003), Area Quantization in quasiextremal black holes.

[36] D. Christodoulou, Phys. Rev. Lett. 25, 1596 (1970), Reversible and irreversible transformations in black hole physics.

[37] J. D. Bekenstein, Phys. Rev. D 7, 2333 (1973), Extraction of Energy and Charge from a Black Hole.

[38] S. Hod, Phys. Rev. D 59, 024014 (1998), Best approximation to a reversible process in black hole physics and the area spectrum of spherical black holes.

[39] J. D. Bekenstein, V. F. Mukhanov, Phys. Lett. B, 360, 7 (1995), Spectroscopy of the quantum black hole.

[40] M. Maggiore, Phys. Rev. Lett., 100, 141301, (2008), The Physical interpretation of the spectrum of black hole quasinormal modes.

[41] L. Motl, A. Neitzke, Adv. Theor. Math. Phys., 7, 307 (2003), Asymptotic black hole quasinormal frequencies.

[42] E. Berti, gr-qc/0411025, Black hole quasinormal modes: Hints of quantum gravity?

[43] E. Berti, K. Kokkotas, Phys. Rev. D, 68, 044027, Asymptotic quasinormal modes of Reissner-Nordstrom and Kerr black holes.

[44] E. Berti, V. Cardoso, K. Kokkotas, H. Onozawa, Phys. Rev. D, 68, 124018 (2003), Highly damped quasinormal modes of Kerr black holes. 
[45] M. Maggiore, Nucl. Phys. B, 429, (1994), Black holes as quantum membranes.

[46] A. Barvinsky, S. Das, G. Kunstatter, Class. Quant. Grav., 18 (2001).

[47] X. Rao, B. Wang, G. Yang, Phys. Lett. B, 649, 472 (2007), Quasinormal modes and phase transition of black holes; X. He, B. Wang, R. Cai, Phys. Lett. B, 688, 230 (2010), Signature of the black hole phase transition in quasinormal modes.

[48] R. D. Fontana, K. Kokkotas, R. Konoplya, On the thermodynamical properties of the quasi-normal spectrum for Reissner-Nordström black holes (in preparation).

[49] J. Jing, Q. Pan, Phys. Lett. B, 660, 13 (2008), Quasinormal modes and second order thermodynamic phase transition for Reissner-Nordström black hole.

[50] R. A. d'Inverno, Introducing Einstein's Relativity, Oxford University Press (1999).

[51] S. Chandrasekhar, The Mathematical Theory of Black Holes, Clarendon Press, Oxford (1992).

[52] F. J. Zerilli, Phys. Rev. D, 9, 860 (1974), Perturbation analysis for a gravitational and electromagnetic radiation in a Reissner-Nordström geometry.

[53] V. Moncrief, Phys. Rev. D, 9, 2707 (1974), Odd-parity stability of a Reissner-Nordström black hole.

[54] B. Zwiebach, Cambridge University Press, A First Course in String Theory (2004).

[55] S. Iyer, C. M. Will, Phys. Rev. D, 35, 3621 (1987), Black Hole Normal Modes: A Wkb Approach. 1. Foundations And Application Of a Hihger Order Wkb Analysis Of Potential Barrier Scattering; K. D. Kokkotas, B. 
F. Schutz, Phys. Rev. D, 37, 3378 (1988), Black-hole normal modes: A WKB approach. III The Reissner-Nordström black hole; E. S. Iyer, Phys. Rev. D, 41, 374 (1990), Black Hole Normal Modes: A Wkb Approach. 4. Kerr Black Holes.

[56] R. A. Konoplya, J. Phys. Stud., 8, 93 (2004), Quasinormal modes of the Schwarzschild black hole and higher order WKB approach.

[57] K. D. Kokkotas, B. F. Schutz, Phys. Rev. D, 37, 3378 (1988), Black-hole normal modes: A WKB approach. III The Reissner-Nordström black hole; E. Berti, K. Kokkotas Phys. Rev. D, 71, 124008 (2005), Quasinormal modes of Kerr-Newman black holes:Coupling of eletromagnetic and gravitational perturbations.

[58] N. Andersson, M. E. Araujo, B. F. Schutz, Class. Quant. Grav., 10, 735 (1993), The phase-integral method and the black hole normal modes.

[59] E. W. Leaver, Proc. Roy. Soc. Lond. A, 402, 285 (1985), An Analytic representation for the quasi normal modes of Kerr black holes.

[60] E. W. Leaver, Phys. Rev. D, 41, Quasinormal modes of ReissnerNordström black holes.

[61] P. C. W. Davies, Class. Quant. Grav. , 6, 1909 (1989), Thermodynamic Phase Transitions of Kerr-Newman Black Holes in De Sitter Spaces.

[62] P. C. W. Davies, Rep. Prog. Phys., 41, 1313 (1979), Thermodynamics of black holes.

[63] H. Kodama, A. Ishibashi, Prog. Theor. Phys. , 111, 29 (2004), Master equations for perturbations of generalised static black holes with charge in higher dimensions.

[64] On the quasinormal modes for cosmological black holes with charges. (In Preparation).

[65] C. Molina, D. Giugno, E. Abdalla, A. Saa, Phys. Rev. D, 69, 104013 (2004), Field Propagation in de Sitter black holes. 
[66] H. Kodama, M. Sasaki, Prog. Theor. Phys. Suppl, 78, 1 (1984), Cosmological perturbation theory.

[67] C. Gundlach, R. H. Price, J. Pullin, Phys. Rev. D, 49, 883 (1994), Late time behavior of stellar collapse and explosions: 1.Linearized perturbations.

[68] E. Abdalla, C. B. M. H. Chirenti, A. Saa, Phys. Rev. D, 74, 084029 (2006), Quasinormal modes for the Vaidya Metric.

[69] P. R. Brady, C. M. Chambers, W. Krivan, P. Laguna, Phys. Rev. D, 55, 7538 (1997), Telling tails in the presence of a cosmological constant.

[70] E. Berti, V. Cardoso, Phys. Rev. D, 77, 087501 (2008), Quasinormal modes and Thermodynamic phase transitions.

[71] B. Wang, C. Molina, E. Abdalla, Phys. Rev. D, 63, 084001 (2001), Evolving of a massless scalar field in Reissner-Nordström-anti-de Sitter spacetimes.

[72] B. Wang, C. Lin, E. Abdalla, Phys. Lett. B, 481, 79 (2000), Quasinormal modes of Reissner-Nordström anti-de Sitter black holes.

[73] B. Wang, E. Abdalla, R. Su, Phys. Rev. D, 62, 047501 (2000), Geometry and Topology of two kinds of extreme Reissner-Nordström-anti-de Sitter black holes. 